ANL/EAIS/TM--2

DE9 0006468

ANL/EAIS TTM-2

\title{
Data Base of Accident and Agricultural Statistics for Transportation Risk Assessment
}

by C.L. Saricks, " R.G. Williams, and M.R. Hopf

Environmental Assessment and Information Sciences Division, Argonne Nationai Laboratory, 9700 South Cass Avenue, Argonne, Illinois 60439

November 1989

Work sponcored ty United States Department of Energy, Assistant Secretary for Nuclear Energy, Office of Civilian Radioactive Waste Management

'Saricks is affiliated with Argonne's Energy Systems Division. 


\section{CONTENTS}

NOMENCLATURE $\ldots \ldots \ldots \ldots \ldots \ldots \ldots \ldots \ldots \ldots \ldots \ldots \ldots \ldots \ldots \ldots \ldots \ldots \ldots \ldots$

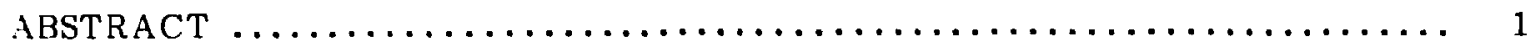

l INTRODUCTION $\ldots \ldots \ldots \ldots \ldots \ldots \ldots \ldots \ldots \ldots \ldots \ldots \ldots \ldots \ldots \ldots \ldots \ldots \ldots$

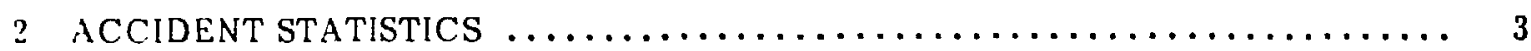

2.1 Highway Transport by Heavy Combination Trucks ............... 3

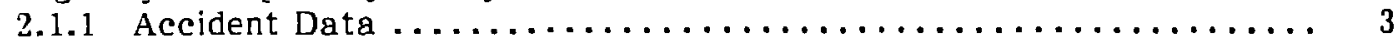

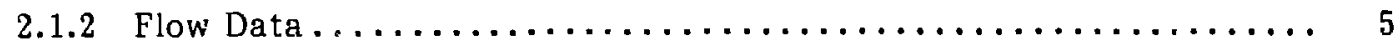

2.2 Rail Transport by Freight Train Railcars $\ldots \ldots \ldots \ldots \ldots \ldots \ldots \ldots \ldots \ldots \ldots$

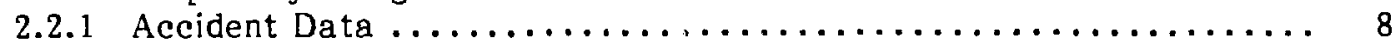

2.2 .2 Flow Data.................................. 8

2.3 Water Transport on Inland Waterways and along the Coasts $\ldots \ldots \ldots \ldots \ldots 10$

2.3 .1 Accident Data ............................... 10

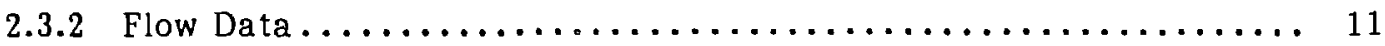

2.4 Summary of Transportation Accident Data................... 11

3 Agricultural Statistics .......................... 20

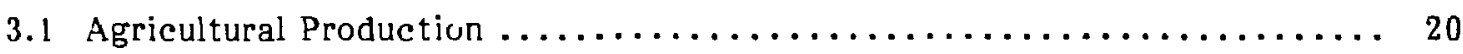

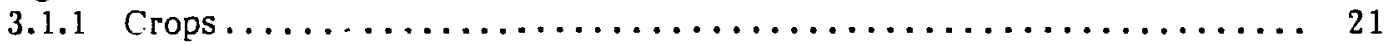

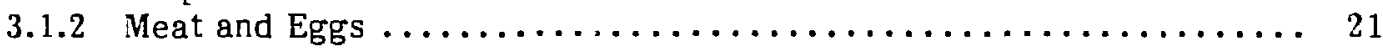

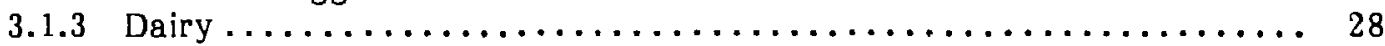

3.2 Per Capita Consumption $\ldots \ldots \ldots \ldots \ldots \ldots \ldots \ldots \ldots \ldots \ldots \ldots \ldots \ldots \ldots \ldots$

3.3 Use of the Agricultural Data in Transportation Accident Modeling ....... 30

4 REFERENCES ................................. 32

APPENDIX A: State Agricultural Production Statistics, $1982 \ldots \ldots \ldots \ldots \ldots \ldots$

APPENDIX B: Agricultural Production Statistics Documentation .......... 55

\section{TABLES}

2.1 State-Specific Multiplier Indexes for Heavy Combination Truck VMT ...... 7

2.2 Reportable Highway Combination Truck Accidents, Fatalities, and Estimated Flows by State and Road Type, $1986 \ldots \ldots \ldots \ldots \ldots \ldots \ldots \ldots$

2.3 Highway Combination Truck Accident, Fatality, and Injury Rates Based on Reportable Accidents, $1986 \ldots \ldots \ldots \ldots \ldots \ldots \ldots \ldots \ldots \ldots$

2.4 Total and Mainline-Only Reportable Railroad Freight Car Accidents, Estimated Railcar-Kilometers for 1986, and Accident Rates for 1986 and 1985-1986 Combined 


\section{TABLES (Cont'd)}

2.5 Freight Vessel Accident and Casualty Involvement Rates on Domestic Waterways of the United States, Including Coastwise Shipping, $1985 \ldots \ldots \ldots \ldots 19$

3.1 Summary of Total State Agricultural Production, $1982 \ldots \ldots \ldots \ldots \ldots \ldots$

3.2 Surnmary of State Agricultural Production for Land in

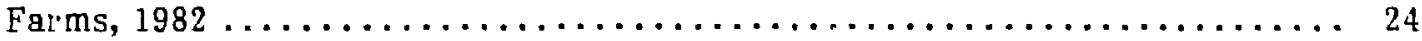

3.3 Summary of State Agricultural Production for Total Land

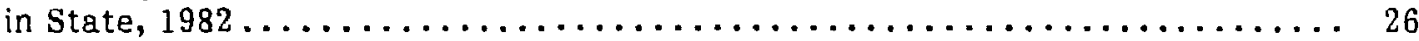

3.4 Annual Per Capita Consumption of Major Food Groups . . . . . . . . . . .. 29

A.1 State Agricultural Production, 1982: Crops $\ldots \ldots \ldots \ldots \ldots \ldots \ldots \ldots \ldots \ldots \ldots . \ldots 37$

A.2 State Agricultural Production, 1982: Meat and Eggs .............. 50

B.1 Documentation for Basic Agricultural Production Statistics ........... 57

B.2 Documentation for Agricultural Production for Land in Farms .......... 59

B.3 Documentation for Agricultural Production for Total Land

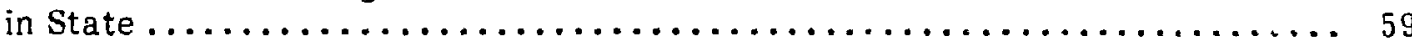




\section{LOMENCLATURE}

The following is a list of the acronyms, initialisms, and abbreviations (including units of measure) used in this document.

\section{ACRONYMS, INITIALISMS, AND ABBREVIATIONS}

$\begin{array}{ll}\text { BMCS } & \text { Bureau of Motor Carrier Statistics } \\ \text { CVCF } & \text { commercial vessel casualty file } \\ \text { DOE } & \text { U.S. Department of Energy } \\ \text { DOT } & \text { U.S. Department of Transportation } \\ \text { EA } & \text { environmental assessment } \\ \text { FAI } & \text { Federal-Aid Interstate } \\ \text { EAI-R } & \text { FAI-Rural } \\ \text { FAI-U } & \text { FAI-Urban } \\ \text { EAP } & \text { Federal-Aid Primary } \\ \text { FARS } & \text { fatal accident reporting system } \\ \text { EAS } & \text { Federal-Aid Secondary } \\ \text { EHWA } & \text { Federal Highway Administration } \\ \text { FRA } & \text { Federal Railroad Administration } \\ \text { HLW } & \text { high-level waste } \\ \text { HPMS } & \text { highway pertormance monitoring system } \\ \text { ICC } & \text { Interstate Commerce Commission } \\ \text { IPF } & \text { iterative proportional fitting } \\ \text { NWPA } & \text { Nuclear Waste Policy Act } \\ \text { OMC } & \text { Office of Motor Carriers } \\ \text { SNF } & \text { spent nuclear fuels } \\ \text { TWS } & \text { truck weight study } \\ \text { USDA } & \text { U.S. Department of Agriculture } \\ \text { VMT } & \text { vehicle-miles of travel }\end{array}$

\section{UNITS OF MEASURE}

$\begin{array}{ll}\text { doz } & \text { dozen } \\ \mathrm{kg} & \text { kilogram(s) } \\ \mathrm{km} & \text { kilometer(s) } \\ \mathrm{km}^{2} & \text { square kilometer(s) } \\ \mathrm{lb} & \text { pound(s) } \\ \mathrm{yr} & \text { year(s) }\end{array}$




\title{
DATA BASE OF ACCIDENT AND AGRICULTURAL STATISTICS FOR TRANSPORTATION RISK ASSESSMENT
}

\author{
by \\ C.L. Saricks, R.G. Williams, and M.R. Hopf
}

\begin{abstract}
A state-level data base of accident and agricultural statistics has been developed to support risk assessment for transportation of spent nuclear fuels and high-level radioactive wastes. This data base will enhance the modeling capabilities for more route-specific analyses of potential isks associated with transportation of these wastes to a disposal site. The data base and methodology used to develop state-specific accident and agricultural data bases are described, and summaries of accident and agricultural statistics are provided.
\end{abstract}

\section{INTRODUCTION}

The Nuclear Waste Policy Act (NWPA) of 1982 established a process for selecting a geologic repository site for the disposal of spent nuclear fuel (SNF) and high-level radioactive waste (HLW). Following the nomination of three potential geologic repository sites, the U.S. Department of Energy (DOE) published environmental assessments (EAs) for the sites in 1986 (DOE 1986a, 1986b, 1986c); these EAs included potential issues raised by public commenters (Appendix $C$ of each EA). One issue of concern is the risk associated with transportation of SNF and HLW to the nominated sites -- in particular, the lack of route-specific risk analyses in the EAs and the generic nature of the RADTRAN III computer code used for the analyses (Madsen et ai. 1986). In addition, it was determined that the agricultural inputs to the model were inadequate.

To address tirace public concerns, the DOE Office of Civilian Radioactive Waste Management has initiated work to enhance capabilities for more route-specific risk analyses. This work involves two major tasks: ( 1 ) enhancement of the data base and (2) enhancement of methodology. This report summarizes a data base of accident and agricultural statistics recently developed for the first task.

In the EAs, accident-related risks for the national transportation system were computed using a single set of accident rates for the entire country. To account for the differences in accident patterns across the country, state-specific accident data are developed in this report. Within each state, accident data are separated for different road types according to the Federal-Aid Administrative Classification System, i.e., 
federal-aid interstate (FAI), federal-aid prinary (FAP), and federal-aid secondary (FAS). Transportation accident rates (probabilities per shipment-kilometer) are calculated on the basis of these disaggregated data.

One of the important radiation exposure pathways considered in the EAs was the ingestion of contaminated foodstuffs resulting from dispersion and subsequent uptake of radioactive materials following transportation accidents. In the EAs, ingestion doses were computed using a generic approach; agricultural productivity for different types of food and for different regions of the United States were not considered. To adequately represent agricultural production patterns for route-specific analyses and to better approximate potential doses from the ingestion pathway, state-level agricultural data are developed in this report. These data include dairy, meat, and crop production data as well as land area in production for each state. 


\section{ACCIDENT STATISTICS}

A prevalent theme in the comments received on the EAs for the candidate geologic repository sites was that data from national sources or highly aggregated sources were used to develop risk parameters for the comparative analysis. In particular, a single national accident rate for shipments of nuclear materials, based on over 10 years of historical information about such shipments, was used to assess potential transportation risks for all projected SNE shipments through all host and transit states. Many commenters expressed dissatisfaction with this apparent simplification and requested that DOE use actual state-level data in future assessments.

This chapter describes the data base and methodology used to develop a statespecific accident data base for future transportation risk assessment. The purpose is to provide a data base that can be used by a risk computer code to differentiate risk according to the location of populations along shipping routes. The procedure that is being applied to the problem of developing state accident rates is described in Sections 2.1, 2.2, and 2.3. These rates have been developed to be appropriate to the conveyarice medium that will be used to transport spent fuel casks, i.e., heavy combination trucks, railears, and water vessels.

\subsection{HIGHWAY TRANSPORT BY HEAVY COMBINATION TRUCKS}

\subsubsection{Accident Data}

Past risk estimation procedures for shipments of nuclear materials have focused on data specific to accidents and incidents involving hazardous and nuclear materials. In the current effort, the recent accident record for the type of vehicle that will be involved in highway transport of spent fuel casks under the NWPA repository program -i.e., heavy combination trucks -- was examined as an appropriate indicator of risk. Although it may be assumed that trucks carrying spent fuel will be subject to safety precautions greater than the average, the current analysis is conservative in that neither the type of cargo nor the lading status of the truck (i.e., loaded or empty) is a factor and additional safety precautions are disregarded. Risk of highway transportation is therefore defined as the number of accident involvements* of heavy combination trucks in year $i$ pe: unit of travel by heavy combination trucks in year $i$ (if travel units are reported or estimated in miles, they are converted to kilometers). The rate is therefore a fractional value. Moreover, both loaded and unloaded truck travel is included in the denominator. Further, it was determined that this unit should be disaggregated within state by road type according to the Federal-Aid Administrative Classification System (i.e., EAI, FAP, and FAS road types), which is the basis of the road maintenance and improvement funding formulas under the Federal-Aid Highwoy Ant. Such disaggregation

\footnotetext{
*An accident involvement is a truck-unit multiplier; for example, the collision of two trucks in a single accident represents two accident involvements.
} 
not only enhances estimation of within-state (between class) variance, to complement the depiction of between-state (nonstratified) variance, but also introduces into the data base a surrogate for objective risk itself (i.e., differences among the systems with respect to average roadway standards and permitted extremes in geometrics).

State-level data, which are readily available, were processed for the numerators of the risk rates (i.e., total counts of accident involvements by road type). The procedure for estimating the denominator was less straightforward but almost equally reliable, as described in Section 2.1.2.

The primary source of heavy combination truck accident data is the 50-T Master File of Accidents of Motor Carriers of Property, compiled annually from carriersubmitted reports by the Office of Motor Carriers (OMC), formerly the Bureau of Motor Carrier Statistics (BMCS), of the U.S. Department of Transportation (DOT). The 50-T file is the only comprehensive central source of public-use, easily accessed data covering heavy combination truck accident involvements by year for all states. All but a few of the accidents recorded in this file each year involve some configuration (bobtail to "turnpike triple") of heavy combination truck. Reporting requirements in the past have stipulated that carriers must report any accident in which damage to property of $\$ 2,000$ or more occurred (reporting of less costly accidents was at the carriers' discretion). Subsequently, the reporting threshold was raised to $\$ 4,200$ in $1986, \$ 4,400$ in 1987 , and $\$ 4,900$ in 1988 .

Another source of data covering what are generally the most severe accidents involving heavy trucks is the fatal accident reporting system (FARS), compiled by calendar year by DOT's National Highway Traffic Safety Administration. This file contains a record of every accident in which a fatality is reported. The principal source of these data is accident reports prepared by civil protection authorities. Because such reports generally provide accurate, detailed data for any accident in which a fatality has occurred, the FARS contains the most comprehensive, nationally available descriptions of accident involvements and consequences not subsequently investigated by the National Transportation Safuty Board (whose reports are even more thorough and detailed). Each record contains information on vehicle deformation, fire occurrence, jackknife, and rollover situations not generally available in the OMC file; in addition, accident location information by road type is usually more precise. However, the subset of heavy truck accidents contained in the FARS file is not sufficiently unbiased to expand to, or even make reasonable inferences about, the full spectrum of truck accident involvements.

The OMC 50-T public-use file for 1986 was selected as the recommended source for the numerator of the accident unit-risk factor for over-the-road SNF inipments because of its exclusive focus on commercial truck traffic and its high degree ui representation of accidents involving such traffic, and because the perioul covered was the most recent (i.e., representative) that was aiso complete at the time the task described herein was underiaken. The FARS file for 1985 was selected as the recommended source of the numerator for the nonradiological fatality unit-risk factor because it corresponds to the 1986 50-T record in both timeliness and completeness, but with respect to fatal accidents only. Processing of a single-year cross section as the initial data base for rate estimation accomplished two desired ends: (1) use of data only from a time period representative of post-recession, post-deregulation conditions (i.e.. after 
1980) in the road transport industry and of the essential completion of the interstate system, and (2) inclusion of the most recent available consistent set of data believed to be complete for the time period covered.

The method of Peterson and Southworth (1984) for estimating state-level truck flows (see Section 2.1.2) represents such a fundamental improvement of the estimation procedure for the state-level risk factor denominator that a commensurate improvement in quality assurance had to be applied to the numeraior. Therefore, a state-by-state listing of the salient componei:ts of each of the 23,000 heavy truck accident involveinent records in the 1986 OMC 50-T master file was generated through the Statistical Analysis System $\left(\mathrm{SAS}^{\otimes}\right.$ ) in order to manually assign the accident location provided on each record to the relevant administrative category (FAI-Urban, FAI-Rural, FAP, FAS, or none of the above). This in turn necessitated state-by-state transcription of the FAP system overlay from a national map of that system provided by the regional office of the Federal Highway Administration (FHWA). Although this method is rather cumbersome, it was determined to be at least $50 \%$ more accurate than the method used in a pilot study for this task, in which accident records were tabulated according to the road identifier preface of the coded location (assuming that the 50-T records are complete and authentic). Moreover, it enabled efficient "cleanup" of misassigned or miscoded records, which could not te accomplished under the pilot method. It is therefore planned to repeat this procedure during fiscal years 1989-1992 using annual OMC data for the period $1987-1990$.

\subsubsection{Flow Data}

To construct a denominator for the estimate of road accident/fatality risk per shipment-kilometer, it was necessary to develop estimates of total kilometers (converted from miles) of heavy combination truck travel by state per unit time. The following factors must also be considered: (1) a proportion of truck travel is dead-head mileage in which no actual shipment is involved and (2) a truck's accident risk is largely independent of its cargo. Therefore, for both highway and rail activity, all relevant movements, loaded or empty, on the systems of interest are included in the denominator.

In its annual publication Highway Statistics, the FHWA (1987) reproduces a series of tables that present the results of data analyses performed using fuel sales data reported by states, vehicle counts by type at official 24-hour counting stations across the country, and data from studies such as the Truck Inventory and Use Survey (U.S. Bureau of the Census 1982) regarding vehicular usage patterns and fuel economy. These tables report the following categories:

- Category A -- Estimated vehicle-miles of travel (VMT) by vehicle type on urban and rural roadways,

- Category B -- VMT for all highway vehicles (combined) by state and road functional classification, and

- Category C -- V.MT for highway vehicles by state and federal-aid classification. 
In order to supply the denominator needed to compute each unit-risk fa:tor for road accident involvements, the FHWA data are used to allocate the appropriate share of the "known" national total V:MT by heavy combination trucks in Category A to earh state.

Currently. no actual flow data (total truck movements $\times$ miles of road by road type) are available in either state- or national-level summaries -- except as estimated by the FHWA in modeling efforts (not yet certified). According to count station data, a high proportion of heavy combination truck flow (truck share of total miles not known) takes place on the interstate highway system, and this flow is generally higher than the national average in states with one or both of the following characteristics:

- Category 1 -- A large number of commercial and industrial origins and destinations, and/or

- Category 2 -- An interposed location with respect to the major national commodity flow vectors.

Systematic identification and appropriate weighting of these states have heretofore been achievable only through ad hoc methods with statistically weak justification. Recently, however, Peterson and Southworth (1984) have circumvented this problem in their development of a procedure (originally designed to project transportation fuel use) that generates improved estimates of travel miles by vehicle type at the state level.

This procedure begins with the FHWA summaries of the previous year's statelevel VMT by functional classification (Category B) and national VMT by vehicle type (Category A). However, it also systematically takes into account information developed by FHWA's ongoing truck weight study (TWS) and highway performance monitoring system (HPMS) file of traffic counting data by type (19 total vehicle classes) and time period; these data are collected by individual states at count stations, several hundred of which are in operation.

The siting of these count stations on each of the eight functional classes of roadway is not random and may, in fact, introduce a bias in favor of relatively higher truck counts. However, the values for Category B (state functional-class VMT data) and Category A (vehicle-type VMT data) may be entered as control margins in an iterative proportional fitting (IPF) cell-filling algorithm using log-linear adjustment techniques (Willekens 1985). This ensures that no published VMT totals (by state or vehicle type) are violated and that each applicable road/vehicle type combination is assigned a nonzero value of VMT within a state (for example, no "rural interstate" road miles exist in Delaware, so no VMT would be assigned to that functional classification there). The resulting VMT matrix can then be used in a subsequent IPF routine for which the control margins are obtained from Category $\mathrm{C}$ (state federal-aid VMT) data.

Peterson and Southworth's method expands the three-dimensional matrix of V.MT by state, functional class, and administrative class to four dimensions (vehicle information added) using vehicle type proportions from TWS/HP.MS count station data. The latter matrix is then collapsed back to three dimensions, dropping Category $B$ but forcing consistency with all totals in Category $A$ and Category $C$ (which has the desired 
effect of systematically modifying heavy truck count shares within state and administrative class upward or downward so that they become actual VMT shares). As expected, this increases light and heavy truck shares in corridor states and less densely populated regions and reduces them in the most densely populated states (where truck trip lengths are lower on the average).

Analysis of the results of Peterson and Southworth (1984) yields approximate state-specific indexes for heavy combination truck VMT, as shown in Table 2.1. Although it is feasible to use these new multipliers in future year computations, it would be far more consistent to iepeat Peterson and Southworth's procedure with the most recent year's data to determine whether or not state-level rates are stationary. Otherwise, an assumption that heavy truck activity is growing at a uniform national rate applicable within every state would be implicit; based on past data, such an assumption is nôt tenable.

TABLE 2.1 State-Specific Multiplier Indexes for Heavy Combination Truck VMT

\begin{tabular}{|c|c|c|c|c|c|}
\hline State & Index ${ }^{a}$ & State & Index ${ }^{a}$ & State & Index ${ }^{a}$ \\
\hline Alabama & 1.7 & Maine & 0.4 & Ohio & 1.5 \\
\hline Arizona & 2.0 & Maryland & 0.5 & Oklahoma & 1.3 \\
\hline Arkansas & 1.0 & Massachusetts & 0.4 & Oregon & 1.2 \\
\hline California & 1.25 & Michigan & 0.5 & Pennsyl vania & 1.1 \\
\hline Colorado & 0.6 & Minnesota & 0.4 & Rhode Isl and & 0.3 \\
\hline Connerticut & 0.7 & Mississippi & 0.8 & South Carolina & 1.0 \\
\hline Delaware & 1.7 & Missouri & 0.9 & South Dakota & 1.0 \\
\hline Elorida & 0.7 & Montana & 2.5 & Tennessee & 1.0 \\
\hline Georgia & 1.5 & Nebraska & 1.0 & Texas & 1.0 \\
\hline Idaho & 0.75 & Nevada & 0.75 & Utah & 1.0 \\
\hline Illinois & 1.0 & New Hampshire & 0.75 & Vermont & 0.3 \\
\hline Indiana & 1.4 & New Jersey & 0.4 & Virginia & 1.25 \\
\hline Iowa & 1.8 & New Mexico & 1.7 & Washington & 1.1 \\
\hline Kansas & 0.9 & New York & 0.6 & k'est Virginia & 0.7 \\
\hline Kentucky & 1.3 & North Carolina & 0.8 & Wisconsin & 0.5 \\
\hline Louisiana & 1.25 & North Dakota & 0.7 & Wyoming & 2.0 \\
\hline
\end{tabular}

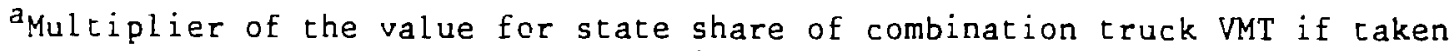
as the computed value of the state's share of total VMT for 1986. 


\subsection{RAIL TRANSPORT BY FREIGHT TRAIN RAILCARS}

\subsubsection{Accident Data}

As for highway transport, it was deemed appropriate for rail transport to examine the types of railroad shipments that will be involved in relocation of spent fuel. Spent fuel casks will move over railroad routes in sections of one, two, three, or more railcars, up to and including unit (dedicated) trains. Therefore, although the propulsion medium for rail shipments is the locomotive, the conveyance (haulage) medium is the railcar, which has no independent power source and may bear the consequences of forces originating from elsewhere in the train. The rail parallel to the numerator jor the truck accident unit-risk factor is total reportable railcar accident involvements by state per unit time. In this case, distinctions may be drawn from available data about the type of track on which an accident occurred (main, yard, siding, or industrial); however, similar partitioning according to track speed class (an index of track physical condition) is very difficult because this parameter is incomplete in accident records and does not appear at all in flow data. Track class may not be a significant variable (Battelle Memorial Institute 1989).

For railroads, reliable sources of empirical information and inference exist for both accidents and fatalities (numerator) and railcar-kilometers (denominator). The source for the numerator is the annual Accident/Incident Bulletin compiled from carrier reports by DOT's Federal Railroad Administration (FRA) Office of Safety. This file (available on tape in a public-use version and supplemented by a published annual summary of statistics) contains comprehensive and internally consistent descriptors for each accident, including the state of occurrence. The file is ahecked by the FRA for duplicate entries (more than one railroad involved in an accident), which are eliminated. By definition, an accident is a collision, derailment, or other event involving the operation of railroad on-track equipment resulting in damages that exceed the reporting threshold (\$4,900 in 1985 and 1986). The 1985 and 1986 files are the most recent complete annual records available for this study, and they have therefore been used for a one-year (1986) and two-year (1985-1986) cross-sectional estimation of rates.

\subsubsection{Flow Data}

The unit-risk factor denominator based on flow data for rail shipments is total railcar-kilometers moved per unit time by state, yielding the desired ratio of accident involvements per shipment-kilometer. Because rail shipments move on dedicated, privately owned networks, they are mucn more closely monitored by the carriers themselves through the manifests or waybills required for each shipment. That is, the commodity, number of cars, and actual routing of the shipment are entered into a computer file along with other data. The Interstate Commerce Commission (ICC) requires that all railroads annually report on these shipments by submitting a representative, quality-checked sample of waybills (nominally 1\%, but actually higher). The ICC then processes these records into both proprietary data (carriers, origin, and destination points identified) and public-use date (origin, interchange, and destinaticn 
states only identified). The public-use file for an avarage year contains well over 300,000 records; each record is drawn from a specific waybill and includes origin and destination regions, rail miles of the shipment, and (expanded) number of railcars loaded for that shipment.

Expansion factors are applied to obtain a reliable estimate of the total (loaded) shipments in a given year. In its annual handbook, Railroad Facts, the Association of tmerican Railroads $(1986,1987)$ reports the proportion of railcar-miles in the preceding year that were actually involved in shipments; by converting the waybill sample's railcarmiles to railcar-kilometers and dividing ty this proportion (usually 50 to 60\%), total railcar-kilometers by origin and destination state are obtained.

Finally, these values (cars $\times$ kilometers) are assigned to the appropriate origin, destination, and intervening states. Shipments with origin and destination in the same state require only summetion of the (factored) car-kilometers. For interstate shipments, a procedure developed for an earlier project on freight network flow forecasting was utilized. A fully coded version of the FRA national rail network is on file at Argonne National Laboratory; this code includes link-specific movement/impedance costs by commodity type. The impedances appropriate for a typical commodity shipment of high value (but not premium service) were applied to develop a set of most probable flow vectors for all interchanges among each of the 48 states (plus the District of Columbia). These vectors provide length information as well as actual fractions of each state-tostate vector on the rail network that are accounted for by the origination, intervening, and destination states. Thus, an algorithm already constructed to manipulat. such vectors was used to allocate each cell total of the state-to-state origin/destination matrix for year 1985 car-kilometers across all appropriate states. This total was multiplied by the corresponding set of fractions and all state fractional allocations were summed into a car-kilumeter total by state. This final set of 49 state totals became the denominators for the $1985 \mathrm{r}$ isk estimates.

Beginning with the 1986 waybill sample, the ICC no longer provides state origin and destination data in the public-use file but includes such information only at the more aggregated district level of the Bureau of Economic Analysis. This is apparently due to a confidentiality requirement on public-use data; with the recent increase in railroad mergers and consolidations, scme states are effectively served by only one rail carrier, and state-level data therefore become carrier-specific data, which remain proprietary. in order to develop 1986 unit-risk denominators consistent with the 1985 assignment procedure, it was necessary to use a secondary source of waybill information, namely, Railroad Traffic in the United States, a compendium of statistics developed from the waybill sample and published annually by ALn Associates $(1986,1988)$. Schematics of inierstate railcar movements presented in the compendium, in conjuction with carrovement data from the edition of Railroad Facts covering 1986, served as the basis for expanding the 1985 state-level railcar-kilometer totals to 1986 estimated values. Comparison of 1985 and 1986 flow graphs in these publications enabled a reasonable determination of which states had increased rail traffic, decreased traffic, and no net cnange; expansion factors were applied consistent with both these inferences and with the national control total presented in the 1986 compendium (ALK Associates 1988, p. 43). 


\subsection{WATER TRANSPORT ON INLAND WATERWA YS AND ALONG THE CUASTS}

\subsubsection{Accident Data}

In the context of freight transport by domestic waterways, state borders have no significance. That is, the characteristics of each waterway are independent of the lower political jurisdictions through which it passes because a single national entity, the U.S. Army Corps of Engineers (the Corps), is responsible for maintenance over the entire navigable length of the waterway. Therefore, an administrative decision was made to use each geographic waterway system, rather than each state, as the basis for estimating unit-risk factors for water transport. The Corps manages five such domestic (48-state) waterway systems and the coastwise routes (waterborne within a $32-\mathrm{km}$ limit) of the Atlantic Ocean, Gulf of Mexico, Pacific Ocean, Mississippi River (including the Ohio, Tennessee, Missouri, and Arkansas navigable tributaries), and Great Lakes.

The source of data for barge and other (revenue) marine vessel accidents and damage on the domestic waterway system is the commercial vessel casualty file (CVCF) of the U.S. Coast Guard. Records are continuously compiled from reports of waterway situations in which:

1. Actual physical damage to property exceeds $\$ 25,000$,

2. Material damage affects the seaworthiness, maneuverability, or efficiency of a vessel,

3. A vessel is stranded or grounded (with or without damage),

4. Loss of life occurs, and/or

5. Injury occurs causing any person to remain incapacitated for more than 72 hours, except injury to harbor workers not resulting in death and not resulting from vessel casualty or vessel equipment casualty.

The records include vessel characteristics, event, cause, total vessels/crafts involved in the incident, fatalities/injuries, and monetary damage; specific vessel codes indicate whether the vessel was carrying hazardous cargo (U.S. Coast Guard 1984).

The 1985 CVCF was used in developing the numerators for waterborne unit-risk factors because, although the file records now extend well into 1988, the most recent waterway flow data available for use in development of the denominators date from 1985. All 1985 casualty recorós for the following vessel activities were included: break-in-bulk hauls, barge tows, bulk solid cargoes, oil tankerages, container hauls, roll-on roll-off cargoes, unelassified freight hauls, combination (mixed) freight hauls, gas carriers, ore or bulk oil hauls, offshore transfers, bulk liquid chemical hauls, and other unclassified or unknown freight.

All casualties for each waterway were tabulated, and total fatalities and injuries were extracted from the output records. Also, the total vessel involvement in each 
situation (of ten greater than the casualty count) was obtained from each record in order to estimate the likelihood of a craft's involvement in accident-related waterway delays, even if no reportable damage was sustained by that vessel.

\subsubsection{Flow Data}

Waterway movement statistics were obtained from the Corps' most recent annual report on domestic waterborne commerce, which covers calendar year 1985 (U.S. Army Corps of Engineers 1987). Movement statistics are reported in tons and ton-miles by waterway; in orcer to convert these values to units consistent with those used in the highway and rail transport analysis, ton-miles were first converted to ton-kilometers by multiplication, then divided by a barge shipment weight that would be representative of future spent fuel movements in large casks. Tobin, Meshkov, and Jones (1985) have estimated this value to be about 500 short tons. Thus, dividing each waterway's ton-kilometer total by 500 yields the shipment-kilometer denominator needed for computing the unit-risk factor.

\subsection{SUMMARY OF TRANSPORTATION ACCIDENT DATA}

Table 2.2 presents highway data extracted from the OMC 50-T file for 1986, the results of Peterson and Southworih's allocation method, and fatality information from the 1985 FARS file. The values in columns 2 through 6 and 7 through 11 were divided, cespectively, by the values in columns 12 through 16 and a corresponding set of valus that were normalized to the total 1985 national heavy combination truck VMT (except as noted) to yield quotients in units expressible in shipment-kilometers. Table 2.3 shows the resulting accident, fatality, and injury rates per kilometer according to the Federal-Aid Administrative Classification System; the magnitudes are $10^{-7}, 10^{-8}$, and $10^{-7}$, cespectively (the injury rates were derived from injury counts in the OMC file).

Table 2.4 shows the corresponding data from the 1986 FRA Accident/Incident Bulletin (DOT 1986) and the 1985 ICC waybill sample public-use tape, expanded to 1986 as described above, and the resulting one- and two-year rates for railcar accidents by state. These rates are reported for total accidents (main plus siding plus yard) and mainline-only accidents involving railcars on carrier property. The great majority of railcar accidents in many states occur in railyards (therefore at low speeds) and, even when all accidents are counted, the rate for rail operations $p: r$ unit shipping distance is ronsistently less than for truck operations. Moreover, fatalities due to railroad accidents are so iow that a singla value is applied for all operations.

rable 2.5 presents first-level estimates of vessel accident and casualty involvement counts and unit-risk factors by waterway. The sources of these estimates are the 1985 Coust Guard casualty file and the Corps data on waterborne commerce. As with rail, fatalities and injuries are relatively infrequent in domestic waterway moveinents, perhaps due to the low speed of the crafts involved. However, the probability of a vessel accident involvement is significantly higher than sustaining reportable damage or any other casualty, especially on the relatively congested Mississippi River and Gulf of Mexico waterway systems. 
TARl,F 2.2 Reportable Highway Combination Truck Aceidents, Futalities, and Estimated Hlows by State and Road Type, $1986^{\mathrm{B}}$

\begin{tabular}{|c|c|c|c|c|c|c|c|c|c|c|c|c|c|c|c|}
\hline \multirow[b]{2}{*}{ State } & \multicolumn{2}{|c|}{ Combination } & \multicolumn{3}{|c|}{ Truck Accidents } & \multicolumn{5}{|c|}{ Truck Accident Fatalicies } & \multicolumn{2}{|c|}{ Estimaled Heavy } & \multicolumn{2}{|c|}{ Truck Traffic $\left(10^{6}\right.$} & \multirow{2}{*}{$\frac{\mathrm{km} \text { ) }}{\text { EAS }}$} \\
\hline & FAI -U & FA $[-R$ & FAI & FAP & FAS & $\mathrm{EAI}-\mathrm{U}$ & EAI - R & FAI & FAP & FAS & FAI $-U$ & FAI $-R$ & FAI & FAP & \\
\hline Al abama & 78 & 98 & 176 & 228 & 49 & 8 & 20 & 28 & 45 & 0 & 700.5 & 978.5 & 1679.0 & 1491.5 & 386.2 \\
\hline Arizuna & 39 & 142 & 181 & 49 & 10 & 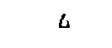 & 49 & 53 & 23 & 7 & 295.3 & 1750.8 & $2 . \therefore 6.1$ & 680.4 & 109.5 \\
\hline Arkansas & 57 & 108 & 165 & 195 & 34 & 4 & .8 & 22 & 79 & 6 & 135.7 & 381.6 & 517.3 & 619.2 & 70.0 \\
\hline Cal ifornia & 223 & 170 & 393 & 307 & 16 & 76 & 52 & 128 & 218 & 40 & 1742.1 & 9810.2 & 11552.3 & 4572.1 & 772.4 \\
\hline Colorado & 56 & 62 & 118 & 83 & 17 & 6 & 25 & 31 & 31 & 4 & 253.8 & 344.6 & 598.4 & 476.6 & 43.9 \\
\hline Connecticut & 165 & 44 & 209 & 39 & 11 & 7 & 7 & 14 & 13 & 2 & 585.4 & 225.1 & 810.5 & 164.6 & 37.4 \\
\hline De Laware & 21 & - & 21 & 37 & 4 & 2 & - & 2 & 10 & 0 & 151.1 & 0 & 151.1 & 261.8 & 55.0 \\
\hline Florida & 118 & 102 & 220 & 301 & 43 & 9 & 31 & 40 & 3.24 & 25 & 896.6 & 1154.2 & 2050.8 & 1581.0 & 152.4 \\
\hline Ceorgia & 192 & 184 & 376 & 273 & 44 & 14 & 27 & 41 & 114 & 19 & 1455.3 & 1941.6 & 3396.9 & 1362.0 & 385.9 \\
\hline Idano & 9 & 43 & 52 & 40 & 7 & 0 & 8 & 8 & 16 & 3 & 28.2 & 201.2 & 229.4 & 152.2 & 66.2 \\
\hline Illinois & 472 & 186 & 658 & 317 & 13 & 12 & 34 & 46 & 79 & 7 & 1547.7 & 1403.6 & 2951.3 & 1301.1 & 129.9 \\
\hline Indiana & 273 & 172 & 445 & 378 & 35 & 5 & 25 & 30 & 72 & 21 & 382.4 & 1485.3 & 1867.7 & 1319.5 & 419.8 \\
\hline Iowa & 40 & 124 & 164 & 139 & 16 & 1 & 4 & 5 & 44 & 4 & 223.1 & 733.8 & 956.9 & 914.2 & 336.8 \\
\hline Kansas & 57 & 63 & 120 & 177 & 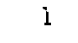 & 2 & 7 & $?$ & 56 & 4 & 131.6 & 396.3 & 527.9 & 569.5 & 80.5 \\
\hline kentucky & 37 & 110 & 197 & 107 & 55 & 8 & 27 & 35 & 52 & 20 & 329.9 & 1051.7 & 1381.6 & 564.8 & 464.2 \\
\hline Louisiana & 75 & 78 & 153 & 146 & 30 & 3 & 16 & 19 & 60 & 28 & 402.9 & 1029.9 & 1472.8 & 629.6 & 258.1 \\
\hline Maine & 14 & 22 & 36 & 32 & 4 & 0 & 1 & 1 & 7 & 4 & 12.7 & 116.1 & 128.8 & 103.5 & 29.5 \\
\hline Maryl and & 154 & 76 & 230 & 141 & 19 & 9 & 6 & 15 & 40 & 5 & 327.9 & 465.3 & 793.2 & 383.9 & 28.6 \\
\hline MassachusetLs & 53 & 53 & 106 & 43 & 25 & 6 & 0 & 6 & 16 & 2 & 486.2 & 222.6 & 708.8 & 315.3 & 45.7 \\
\hline Michigan & 179 & 98 & 277 & 175 & 3 & 19 & 17 & 36 & 48 & 13 & 395.9 & 909.1 & 1305.0 & 645.7 & 195.4 \\
\hline Minnesota & 45 & 54 & 99 & 130 & 17 & 1 & 7 & 8 & 50 & 3 & 96.8 & 194.5 & 291.3 & 473.0 & 64.2 \\
\hline Mississippi & 33 & 55 & 88 & 187 & 11 & 3 & 25 & 28 & 61 & 18 & 71.9 & 369.7 & 441.6 & 385.7 & 49.3 \\
\hline Missourl & 173 & 148 & 321 & 236 & 34 & 21 & 16 & 37 & 63 & 10 & 296.5 & 770.0 & 1066.5 & 1022.1 & 173.9 \\
\hline Muntana & 18 & 48 & 66 & 53 & 3 & 1 & 9 & 10 & 20 & 1 & 18.2 & 492.7 & 510.9 & 630.6 & $0 . .7$ \\
\hline Nebraska & 21 & 56 & 77 & 69 & 2 & 0 & 10 & 10 & 26 & 0 & 50.0 & 308.0 & 358.0 & 354.8 & 64.9 \\
\hline Ne:ada & 19 & 42 & 61 & 24 & 5 & 0 & $1 ?$ & $1 ?$ & 5 & 1 & 53.7 & 237.0 & 2901.7 & 98.9 & 29.1 \\
\hline
\end{tabular}


TABLE 2.2 (Cont'd)

\begin{tabular}{|c|c|c|c|c|c|c|c|c|c|c|c|c|c|c|c|}
\hline \multirow[b]{2}{*}{ Stace } & \multicolumn{2}{|c|}{ Combination } & Truck & \multicolumn{2}{|c|}{ Accidents } & \multicolumn{5}{|c|}{ Truck Accident Fatalities } & \multicolumn{2}{|c|}{ Est imated Heavy } & \multicolumn{2}{|c|}{ Truck Trafic $\left(10^{6}\right.$} & \multirow{2}{*}{$\frac{\mathrm{km})}{\mathrm{FAS}}$} \\
\hline & FAI-U & $F A I-R$ & FAI & FAP & FAS & FAI-U & FAI-R & FAI & FAP & FAS & $F A I-U$ & $F A I-R$ & FAI & FAP & \\
\hline New Hampshire & 0 & 6 & 6 & 26 & 7 & 1 & 0 & 1 & 9 & 0 & 38.9 & 177.2 & 216.1 & 149.0 & 31.0 \\
\hline Now Jersed & 140 & $1 \therefore 9$ & 289 & 214 & 24 & 18 & 3 & 21 & 38 & 11 & 735.8 & 134.3 & 870.1 & 419.3 & 96.3 \\
\hline New Mexicu & 24 & 97 & 121 & 57 & 7 & 4 & 23 & 27 & 37 & 6 & 111.2 & 746.4 & 857.6 & 378.5 & 75.3 \\
\hline New York & 212 & 211 & 423 & 217 & 74 & 23 & 19 & 42 & 59 & 11 & 1593.4 & 812.1 & 2405.5 & 1152.8 & 36.0 \\
\hline North Carulina & 111 & 133 & 244 & 287 & $9 ?$ & 20 & 18 & 28 & 87 & 52 & $1 \% 8.7$ & 726.2 & 904.9 & 1133.0 & 602.2 \\
\hline North Dakuta & 3 & 18 & 21 & 24 & 0 & 0 & 2 & 2 & 3 & 0 & 10.5 & 135.6 & 146.1 & 126.2 & 34.8 \\
\hline Ohio & 199 & 397 & 596 & 368 & 101 & 23 & 33 & 56 & 86 & 22 & 2122.6 & 2576.2 & 4698.8 & $2\lfloor 76.3$ & 873.3 \\
\hline Oklahoma & 60 & 74 & 134 & 136 & 25 & 11 & 17 & 28 & 60 & 18 & 437.1 & 797.3 & 1234.4 & 1064.2 & 260.0 \\
\hline Siregon & 36 & 128 & 164 & 130 & 9 & 2 & 7 & 9 & 47 & 8 & 222.9 & 700.5 & 923.4 & 531.0 & 91.2 \\
\hline Pennsylvania & 151 & 565 & 716 & 496 & 53 & 25 & 69 & 94 & 86 & 4 & 624.3 & 2232.9 & 2857.2 & 2015.1 & 308.0 \\
\hline Rhode Island & 13 & 5 & 18 & 5 & 2 & 3 & 0 & 3 & 0 & 0 & 58.8 & 24.3 & 83.1 & 29.4 & 4.1 \\
\hline South Carolina & 22 & 108 & 130 & 177 & 19 & 10 & 12 & 22 & 59 & 13 & 99.1 & 787.9 & 887.0 & 672.7 & 136.0 \\
\hline South Dakota & 0 & 32 & 32 & 41 & 1 & 1 & 4 & 5 & 8 & 0 & 6.7 & 187.0 & 193.7 & 126.7 & 20.8 \\
\hline randesses & 154 & 185 & 339 & 199 & 8 & 17 & 40 & 57 & 55 & 13 & 260.6 & 1184.6 & 1445.2 & 662.9 & 217.7 \\
\hline roxas & 291 & 272 & 563 & 461 & 54 & 68 & 60 & 128 & 237 & 12 & 2315.2 & 2473.5 & 4788.7 & 3901.8 & 843.9 \\
\hline Weat & 23 & 79 & 102 & 45 & 4 & 8 & 7 & 15 & 18 & 3 & 212.5 & 313.8 & 526.3 & 216.7 & 70.2 \\
\hline Vermont & 0 & 12 & 12 & 26 & 4 & 0 & 0 & 0 & 7 & 1 & 5.8 & 67.4 & 73.2 & 80.0 & 26.7 \\
\hline virzinia & 75 & 224 & 299 & 230 & 30 & 10 & 16 & 26 & 61 & 13 & 748.6 & 672.5 & 1421.1 & 1583.1 & 945.1 \\
\hline Washington & 38 & 106 & 144 & 80 & 11 & 5 & 3 & 8 & 48 & 4 & 464.1 & 741.6 & 1205.7 & 691.1 & 669.4 \\
\hline West Vireinia & 25 & 100 & 125 & 130 & 21 & 1 & 7 & 8 & 28 & 2 & 33.0 & 276.3 & 309.3 & 177.8 & 101.7 \\
\hline Wisconsin & 50 & 111 & 161 & 192 & 12 & 4 & 10 & 14 & 58 & 5 & 92.7 & 165.8 & 258.5 & 835.7 & 69.1 \\
\hline Wyoming & 14 & 145 & 159 & 41 & 5 & 0 & 8 & 8 & 8 & 2 & 20.1 & 376.3 & 396.4 & 178.1 & 25.9 \\
\hline
\end{tabular}

acciderts = accident involvements of heavy combination trucks; fatalities = accidenc-related deaths occurring up co 30 days ifter date of arcident; FAI-U = Federal-Aid Interstate highway system in urbanized areas; FAI-R = Federal-Ajd Interstate highway system in rural areas; FAI = Federal-Aid [nterstate highway system (urban and rural combined); FAP = Federal-AidPrimary highway system; Fas = Federal-Aid Secondary (rural) highway system. 
TABLr 2.3 Highway Combination Truek Accident, Patality, and Injury Rutes Based on Reportable Accidents, 1986

\begin{tabular}{|c|c|c|c|c|c|c|c|c|c|c|c|c|c|c|c|}
\hline \multirow[b]{2}{*}{ State } & \multirow{2}{*}{ Truck } & \multicolumn{4}{|c|}{$\begin{array}{l}\text { Accident Involvement Rat } \\
\left(10^{-7} \text { accidents per }\right. \\
\text { shipment-kilometer) }\end{array}$} & \multicolumn{5}{|c|}{$\begin{array}{c}\text { Truck Accident Fatality Rates } \\
\left(10^{-8} \text { fatalities per }\right. \\
\text { shipment-kilometer })\end{array}$} & \multicolumn{5}{|c|}{$\begin{array}{c}\text { Truck Accident Injury Rates } \\
\left(10^{-8} \text { injuries per }\right. \\
\text { shipment-kilometer })\end{array}$} \\
\hline & & FAI-R & FAI & FAP & FAS & FAI-U & $E A I-R$ & FA1 & EAP & FAS & FAI-U & FAI - R & FAI & FAP & FAS \\
\hline Al abama & 1.11 & 1.00 & 1.05 & 1.53 & 1.27 & 1.19 & 2.13 & 1.74 & 3.14 & 0.62 & 1.07 & 1.04 & 1.05 & 1.68 & 1.42 \\
\hline Arizona & 1.32 & 0.81 & 0.88 & 0.72 & 0.91 & 1.41 & 2.92 & 2.70 & 3.52 & 6.66 & 1.33 & 0.75 & 0.85 & 0.91 & 2.65 \\
\hline Arkansas & 4.20 & 2.83 & 3.19 & 3.15 & 4.86 & 3.07 & 4.91 & $4.1: 7$ & 13.29 & 8.93 & 2.14 & 3.70 & 3.31 & 2.70 & 4.43 \\
\hline Cal ifornia & 1.28 & 0.17 & 0.34 & 0.67 & 0.21 & 4.54 & 0.55 & 1.15 & 4.97 & 5.39 & 0.98 & 0.20 & 0.31 & 0.69 & 0.21 \\
\hline Colorado & 2.21 & 1.80 & 1.97 & 1.74 & 3.87 & 2.46 & 7.50 & 5.40 & 6.78 & 9.49 & 1.54 & 2.06 & 1.84 & 1.95 & 2.96 \\
\hline Connecticut & 2.82 & 1.95 & 2.58 & 2.37 & 2.94 & 1.25 & 3.24 & 1.80 & 8.22 & 5.57 & 1.38 & 3.73 & 2.04 & 2.00 & 3.74 \\
\hline Delaware & 1.39 & - & 1.39 & 1.41 & 0.73 & 1.38 & - & 1.38 & 3.98 & 0.62 & 3.64 & - & 3.64 & 1.30 & 0.38 \\
\hline Florida & 1.32 & 0.88 & 1.07 & 1.90 & 2.82 & 1.05 & 2.80 & 2.03 & 8.17 & 17.09 & 1.24 & 0.94 & 1.07 & 1.93 & 2.82 \\
\hline Georgia & 1.32 & 0.95 & 1.11 & 2.00 & 1.14 & 1.00 & 1.45 & 1.26 & 8.72 & 5.13 & 1.05 & 1.27 & 1.17 & 2.14 & 1.01 \\
\hline Idahe & 3.19 & 2.14 & 2.27 & 2.63 & 1.06 & 0.47 & 4.14 & 3.63 & 10.95 & 4.72 & 2.48 & 1.79 & 1.87 & 2.04 & 1.36 \\
\hline [11 inois & 3.05 & 1.33 & 2.23 & 2.44 & 1.00 & 0.81 & 2.52 & 1.62 & 6.32 & 5.61 & 2.60 & 0.95 & 1.81 & 2.37 & 0.85 \\
\hline Indiana & 7.14 & 1.16 & 2.38 & 2.86 & 0.83 & 1.36 & 1.75 & 1.68 & 5.68 & 5.21 & 7.01 & 0.97 & 2.19 & 2.55 & 0.76 \\
\hline Lowa & 1.79 & 1.69 & 1.71 & 1.52 & 0.48 & 0.47 & 0.57 & 0.54 & 5.01 & 1.24 & 1.70 & 1.25 & 1.36 & 1.15 & 0.33 \\
\hline Kansas & 4.33 & 1.59 & 2.27 & 3.11 & 0.12 & 1.58 & 1.84 & 1.78 & 10.24 & 5.18 & 3.34 & 1.72 & 2.12 & 2.21 & 0.13 \\
\hline Kentucky & 2.64 & 1.05 & 1.43 & 1.89 & 1.18 & 2.53 & 2.67 & 2.64 & 9.59 & 4.49 & 3.88 & 0.89 & 1.61 & 1.79 & 1.21 \\
\hline Louisiana & 1.86 & 0.76 & 1.07 & 2.32 & 1.16 & 0.78 & 1.62 & 1.38 & 9.93 & 11.30 & 2.88 & 0.71 & 1.32 & 3.07 & 1.12 \\
\hline Maine & 11.02 & 1.89 & 2.80 & 3.09 & 1,36 & $0.8 ; 7$ & 0.90 & 0.81 & 7.05 & 14.12 & 6.30 & 1.21 & 1.71 & 2.22 & 1.02 \\
\hline Maryland & 4.70 & $1 . \leqslant 3$ & 2.90 & 3.67 & 6.54 & 2.86 & 1.34 & 1.97 & 10.85 & 18.21 & 5.54 & 1.42 & 3.14 & 4.09 & 5.59 \\
\hline Massachusetrs & 1.09 & 2.38 & 1.50 & 1.36 & 6.13 & 1.29 & 0.42 & 0.88 & 5.29 & 4.56 & 0.78 & 1.39 & 0.97 & 1.17 & 5.47 \\
\hline Michigan & 4.52 & 1.08 & 2.12 & 2.71 & 0.15 & 5.00 & 1.95 & 2.87 & 7.74 & 6.93 & 4.07 & 0.75 & 1.75 & 2.60 & 0.15 \\
\hline Minnesota & 4.65 & 2.78 & 3.40 & 2.75 & 2.65 & 1.08 & 3.75 & 2.86 & 11.01 & 4.87 & 3.51 & 1.49 & 2.16 & 2.07 & 1.71 \\
\hline Mississippi & 4.59 & 1.49 & 1.99 & 4.85 & 2.23 & 4.35 & 7.04 & 0.60 & 16.47 & 38.03 & 3.89 & 1.51 & 1.90 & 5.42 & 1.62 \\
\hline Missouri & 3.83 & 1.92 & 3.01 & 2.31 & 1.96 & 7.38 & 2.16 & 3.61 & 6.42 & 5.99 & 6.37 & 1.90 & 3.12 & 2.30 & 1.44 \\
\hline Montana & 9.89 & 0.97 & 1.29 & 0.84 & 0.49 & 5.72 & 1.90 & 2.04 & 3.30 & 1.69 & 4.95 & 0.75 & 0.90 & 0.62 & 0.16 \\
\hline Nebraska & 4.20 & 1.82 & 2.15 & 1.94 & 0.31 & 0.47 & 3.38 & 2.91 & 7.63 & 0.62 & 3.60 & 1.36 & 1.68 & 1.61 & 0.15 \\
\hline Nevada & 3.54 & 1.77 & 2.10 & 2.43 & 1.72 & 0.47 & 5.27 & 4.30 & 5.27 & 3.58 & 2.05 & 1.60 & 1.69 & 1.72 & 2.41 \\
\hline
\end{tabular}


TABLE 2.3 (Cont'd)

\begin{tabular}{|c|c|c|c|c|c|c|c|c|c|c|c|c|c|c|c|}
\hline \multirow[b]{2}{*}{ Slate } & \multirow{2}{*}{$\frac{\text { Truck }}{\text { EAI-U }}$} & \multicolumn{3}{|c|}{$\begin{array}{l}\text { Accigent Involvement } \\
\left(10^{-7} \text { accidents per }\right. \\
\text { shipment-kilometer) }\end{array}$} & Rates & \multicolumn{5}{|c|}{$\begin{array}{c}\text { Truck Accident Patalily Rates } \\
\left(10^{-8} \text { Eatalicies per }\right. \\
\text { shipment-kilameter) }\end{array}$} & \multicolumn{5}{|c|}{$\begin{array}{c}\text { Truck Accident Injury Rates } \\
\left(10^{-8} \text { injuries per }\right. \\
\text { shipment-kilometer) }\end{array}$} \\
\hline & & $F A I-R$ & EAI & FAP & FAS & FAI-iJ & $F A I-R$ & FAI & $\mathrm{FAP}$ & FAS & FAI $-U$ & FAI - R & FAI & FAP & FAS \\
\hline New Ilampshire & 0.82 & 0.34 & 0.28 & 1.74 & 2.26 & 2.68 & 0.42 & 0.48 & 6.29 & 0.62 & 0.69 & 0.09 & 0.11 & 1.88 & 1.29 \\
\hline New Jersey & 1.90 & 11.01 & 3.32 & 5.10 & $3.0 \mathrm{i}$ & 2.55 & 2.33 & $2 .{ }^{5} 1$ & 9.44 & 11.90 & 1.83 & 12.21 & 3.44 & 5.89 & 5.09 \\
\hline New Mexico & $\therefore .16$ & 1.30 & 1.41 & 1.51 & 0.93 & 3.75 & 3.21 & 3.28 & 10.18 & 8.30 & 1.08 & 1.09 & 1.08 & 1.27 & 0.66 \\
\hline New York & 1.23 & 2.00 & 1.76 & 1.88 & 20.50 & 1.50 & 2.44 & 1.82 & 5.33 & 31.83 & 1.05 & 2.17 & 1.42 & 1.48 & 18.61 \\
\hline North Carolina & 0.21 & 1.83 & 2.70 & 2.53 & 1.61 & 5.83 & 2.58 & 3.22 & 7.99 & 8.39 & 6.55 & 1.57 & 2.55 & 2.86 & 1.63 \\
\hline Horth Dakota & 2.86 & 1.33 & 1.44 & 1.90 & $c .12$ & 0.47 & 1.54 & 1.43 & 2.48 & 0.62 & 2.86 & 0.74 & 0.89 & 1.51 & 0.13 \\
\hline Oliso & 0.94 & 1.54 & 1.27 & 1.69 & 1.16 & 1.13 & 1.33 & 1.24 & 4.12 & 2.62 & 0.84 & 1.37 & 1.12 & 1.75 & 1.26 \\
\hline Oklahuma & 1.37 & 0.93 & 1.09 & 1.28 & 0.96 & 2.52 & 2.22 & 2.36 & 5.87 & 7.21 & 1.35 & 0.90 & 1.06 & 1.08 & 0.81 \\
\hline Oregon & 1.62 & 1.83 & 1.78 & 2.45 & 0.59 & 0.93 & 1.04 & 1.02 & 9.22 & 9.14 & 1.57 & 1.20 & 1.29 & 1.88 & 0.44 \\
\hline Pentusylunia & 2.42 & 2.53 & 2.51 & 2.46 & 1.72 & 4.17 & 3.22 & 3.43 & 4.45 & 1.35 & 2.08 & 2.44 & 2.35 & 2.61 & 1.66 \\
\hline Rhode island & 2.21 & 2.06 & 2.17 & 1.70 & 4.88 & 5.31 & 0.42 & 3.76 & 2.48 & 0.62 & 2.72 & 1.65 & 2.41 & 0.34 & 0.13 \\
\hline Souch Carolina & 2.22 & 1.37 & 1.47 & 2.63 & 1.40 & 10.51 & 1.59 & 2.58 & 9.14 & 9.96 & 2.32 & 1.37 & 1.48 & 2.79 & 1.32 \\
\hline South Vakota & 0.82 & 1.71 & 1.65 & 3.24 & 0.48 & 15.55 & 2.23 & 2.69 & 6.57 & 0.62 & 0.67 & 0.70 & 0.69 & 2.60 & 0.13 \\
\hline Tennessee & 5.91 & 1.56 & 2.35 & 3.00 & 0.37 & 6.30 & 3.52 & 4.11 & 8.64 & 6.22 & 5.72 & 1.43 & 2.21 & 3.03 & 0.32 \\
\hline Texas & 1.26 & 1.10 & 1.18 & 1.18 & 0.64 & 3.06 & 2.53 & 2.78 & 6.32 & 8.89 & 1.12 & 0.90 & 1.00 & 1.09 & 0.51 \\
\hline Uitah & 1.08 & 2.52 & 1.94 & 2.08 & 0.57 & 3.92 & 2.32 & 2.97 & 8.65 & 4.45 & 0.94 & 2.36 & 1.79 & 1.85 & 0.28 \\
\hline Vermont & 0.82 & 1.78 & 1.64 & 3.25 & 1.50 & 0.47 & 0.42 & 0.48 & 9.11 & 3.90 & 0.69 & 1.04 & 0.96 & 2.38 & 1.50 \\
\hline virginia & 1.00 & 3.33 & 2.10 & 1.45 & 0.32 & 1.39 & 2.48 & 1.91 & 4.02 & 1.43 & 0.96 & 3.05 & 1.94 & 1.56 & 0.33 \\
\hline Washington & 0.82 & 1.43 & 1.19 & 1.16 & $0.1 E$ & 1.12 & 0.42 & 0.69 & 7.23 & 0.62 & 0.69 & 1.12 & 0.95 & 0.78 & 0.13 \\
\hline best Virginia & 7.58 & 3.62 & 4.04 & 7.31 & 2.06 & 3.16 & 2.64 & 2.69 & 16.40 & 2.05 & 7.58 & 3.22 & 3.69 & 7.71 & 1.47 \\
\hline Wisconsin & 5.39 & 6.69 & 6.23 & 2.30 & 1.74 & 4.49 & 6.28 & 5.64 & 7.23 & 7.54 & 4.32 & 5.73 & 5.22 & 2.11 & 1.59 \\
\hline Wyoming & 6.97 & 3.85 & 4.01 & 2.30 & 1.93 & 0.47 & 2.21 & 2.26 & 4.68 & 8.05 & 0.69 & 3.24 & 3.08 & 1.40 & 0.77 \\
\hline
\end{tabular}


TABI,P 2.4 Total and Mainline-Only Reportable Railroad Freight Car Accidents, Rstimated Railear-Kilometers for 1986, and Accident Rates for 1986 and 1985-1986 Combined

\begin{tabular}{|c|c|c|c|c|c|c|c|}
\hline \multirow[b]{3}{*}{ State } & \multirow{2}{*}{\multicolumn{2}{|c|}{$\begin{array}{c}1986 \text { Accidents } \\
\text { Involving Railcars }\end{array}$}} & \multirow{3}{*}{$\begin{array}{c}1986 \\
\text { Estimated } \\
\text { Railcar- } \\
\text { Kilometers }\end{array}$} & \multicolumn{4}{|c|}{ Rail Freight Accident Rates } \\
\hline & & & & \multicolumn{2}{|c|}{1986} & \multicolumn{2}{|c|}{$\begin{array}{l}1985-1986 \text { Combined } \\
\left(10^{-8} \text { accidents per }\right. \\
\text { railcar-kilometer })\end{array}$} \\
\hline & Total & $\begin{array}{l}\text { Mainline } \\
\text { Only }\end{array}$ & & Total & $\begin{array}{l}\text { Mainline } \\
\text { Only }\end{array}$ & Total & $\begin{array}{c}\text { Mainline } \\
\text { Only }\end{array}$ \\
\hline Al abama & 40 & 17 & $8.51 \times 10^{8}$ & 4.70 & 2.00 & 6.08 & 2.54 \\
\hline Arizona & 24 & 18 & $1.72 \times 10^{9}$ & 1.40 & 1.05 & 2.27 & 1.52 \\
\hline Arkarısas & 39 & 21 & $7.33 \times 10^{8}$ & 5.32 & 2.86 & 7.59 & 4.16 \\
\hline California & 127 & 63 & $2.27 \times 10^{9}$ & 5.59 & 2.77 & 6.94 & 2.99 \\
\hline Colorado & 23 & 16 & $1.39 \times 10^{9}$ & 1.66 & 1.15 & 2.06 & 1.21 \\
\hline Connecticut & 5 & 3 & $1.13 \times 10^{7}$ & 44.12 & 25.47 & 47.06 & 15.69 \\
\hline Del aware & 3 & 1 & $1.09 \times 10^{7}$ & 27.44 & 9.15 & 13.51 & 4.50 \\
\hline District of & & & & & & & \\
\hline Columbia & 0 & 0 & $6.00 \times 10^{5}$ & 1.00 & 0.52 & 80.00 & 0.67 \\
\hline Florida & 25 & 17 & $8.03 \times 10^{8}$ & 3.11 & 2.12 & 4.50 & 2.28 \\
\hline Ceorgia & 80 & 35 & $1.10 \times 10^{9}$ & 7.26 & 3.17 & 7.22 & 3.02 \\
\hline Idaho & 18 & 16 & $3.05 \times 10^{8}$ & 5.90 & 5.25 & 6.23 & 4.43 \\
\hline 111 inois & 395 & 90 & $2.51 \times 10^{9}$ & 15.71 & 3.58 & 15.38 & 3.37 \\
\hline Indiana & 74 & 28 & $1.46 \times 10^{9}$ & 5.07 & 1.92 & 6.75 & 2.51 \\
\hline I owa & 127 & 48 & $2.30 \times 10^{8}$ & 20.17 & 7.62 & 19.71 & 7.89 \\
\hline Kansas & 83 & 42 & $2.16 \times 10^{9}$ & 3.85 & 1.95 & 4.38 & 1.91 \\
\hline Kentucky & 61 & 30 & $1.05 \times 10^{9}$ & 5.83 & 2.87 & 6.37 & 3.38 \\
\hline Louisiana & 61 & 27 & $4.18 \times 10^{8}$ & 14.58 & 6.45 & 17.90 & 5.93 \\
\hline Maine & 11 & 7 & $3.54 \times 10^{7}$ & 31.04 & 19.75 & 42.33 & 19.75 \\
\hline Maryland & 30 & 14 & $3.95 \times 10_{0}^{8}$ & 7.59 & 3.54 & 7.89 & 3.58 \\
\hline Massachusetts & 21 & 10 & $1.70 \cdot 10^{8}$ & 12.33 & 5.87 & 15.42 & 6.82 \\
\hline Michigan & 86 & 35 & $4.04 \times 10^{8}$ & 21.31 & 8.67 & 20.34 & 8.63 \\
\hline
\end{tabular}




\begin{tabular}{|c|c|c|c|c|c|c|c|}
\hline \multirow[b]{3}{*}{ State } & \multirow{2}{*}{\multicolumn{2}{|c|}{$\begin{array}{c}1986 \text { Accidents } \\
\text { Involving Railcars } \\
\end{array}$}} & \multirow{3}{*}{$\begin{array}{c}1986 \\
\text { Estimated } \\
\text { Railcar- } \\
\text { Kilometers }\end{array}$} & \multicolumn{4}{|c|}{ Raii Freight Accident Rates } \\
\hline & & & & \multicolumn{2}{|c|}{1986} & \multicolumn{2}{|c|}{$\begin{array}{l}1985-1986 \text { Combined } \\
\text { ( } 10^{-8} \text { accidents per } \\
\text { railcar-kilometer) }\end{array}$} \\
\hline & Total & $\begin{array}{l}\text { Mainline } \\
\text { Only }\end{array}$ & & Total & $\begin{array}{l}\text { Mairline } \\
\text { Ors y }\end{array}$ & Total & $\begin{array}{l}\text { Mainline } \\
\text { Only }\end{array}$ \\
\hline Minnesota & 144 & 36 & $1.29 \times 10^{9}$ & 11.20 & 2.80 & 11.83 & 2.88 \\
\hline Mississippi & 48 & 34 & $3.03 \times 10_{0}^{8}$ & 15.82 & 11.20 & 13.82 & 8.73 \\
\hline Missouri & 122 & 51 & $2.03 \times 10^{9}$ & 6.02 & 2.52 & 7.61 & 2.75 \\
\hline Montana & 32 & 23 & $2.08 \times 10^{9}$ & 1.54 & 1.11 & 2.05 & 1.34 \\
\hline Nebraska & 82 & 37 & $1.61 \times 10^{9}$ & 5.10 & 2.30 & 5.74 & 2.99 \\
\hline Nevada & 7 & 4 & $2.14 \times 10^{8}$ & 3.27 & 1.87 & 4.19 & 2.96 \\
\hline New llampshire & 1 & 1 & $1.52 \times 10_{8}^{\prime}$ & 6.60 & 6.60 & 22.26 & 19.79 \\
\hline New Jersey & 24 & 4 & $1.51 \times 10_{0}^{8}$ & 15.84 & 2.64 & $16, .88$ & 4.57 \\
\hline New Mexico & 21 & 11 & $2.10 \times 10^{9}$ & 1.00 & 0.52 & 1.11 & 0.67 \\
\hline New York & 52 & 28 & $6.64 \times 10^{8}$ & 7.84 & 4.22 & 10.50 & 5.02 \\
\hline North Carolina & 25 & 12 & $4.45 \times 10_{0}^{8}$ & 5.62 & 2.70 & 5.71 & 2.24 \\
\hline North Dakola & 27 & 18 & $1.15 \times 10^{9}$ & 2.35 & 1.57 & 4.57 & 3.27 \\
\hline ohio & 149 & 60 & $2.08 \times 10^{9}$ & 7.18 & 2.89 & 7.28 & 2.69 \\
\hline OkLahoma & 57 & 30 & $8.96 \times 10^{8}$ & 6.36 & 3.35 & 6.68 & 3.87 \\
\hline Oregon & 51 & 20 & $3.83 \cdot 10_{0}^{8}$ & 13.30 & 5.22 & 13.14 & 5.55 \\
\hline Pennsylvania & 95 & 57 & $1.69 \times 10^{9}$ & 5.63 & 3.97 & 5.53 & 3.46 \\
\hline Rhode Island & 0 & 0 & $2.34 \times 10^{5}$ & 1.00 & 0.52 & 219.30 & 0.67 \\
\hline South Carolina & 16 & 7 & $3.24 \times 10^{8}$ & 4.94 & 2.16 & 6.27 & 3.06 \\
\hline South Dakota & 13 & 10 & $1.59 \times 10^{8}$ & 8.16 & 6.27 & 2.63 & 2.15 \\
\hline Tennessee & 69 & 18 & $1.00 \times 10^{9}$ & 6.87 & 1.79 & 6.33 & 1.64 \\
\hline Texas & 290 & 122 & $3.30 \times 10^{9}$ & 8.79 & 3.70 & 10.39 & 4.03 \\
\hline Ut ah & 13 & 5 & $2.98 \times 10^{8}$ & 4.36 & 1.68 & 5.67 & 2.30 \\
\hline
\end{tabular}




\begin{tabular}{|c|c|c|c|c|c|c|c|}
\hline \multirow[b]{3}{*}{ State } & \multirow{2}{*}{\multicolumn{2}{|c|}{$\begin{array}{c}1986 \text { Accidents } \\
\text { Involving Railcars } \\
\end{array}$}} & \multirow{3}{*}{$\begin{array}{c}1986 \\
\text { Estimated } \\
\text { Railcar- } \\
\text { Kilometers }\end{array}$} & \multicolumn{4}{|c|}{ Rail Freight Accident Rates } \\
\hline & & & & \multicolumn{2}{|c|}{1986} & \multicolumn{2}{|c|}{$\begin{array}{l}1985-1986 \text { Combined } \\
\text { (10-8 accidents pe } \\
\text { railcar-kilometer) }\end{array}$} \\
\hline & Total & $\begin{array}{l}\text { Mainline } \\
\text { Only }\end{array}$ & & Total & $\begin{array}{l}\text { Mainline } \\
\text { Only }\end{array}$ & Total & $\begin{array}{l}\text { Mainline } \\
\text { Only }\end{array}$ \\
\hline Vermont & 3 & 1 & $3.35 \times 10^{7}$ & 8.96 & 2.99 & 19.42 & 13.45 \\
\hline Virginia & 70 & 17 & $1.19 \times 10^{9}$ & 5.86 & 1.42 & 5.48 & 1.76 \\
\hline Washingcon & 36 & 11 & $7.94 \times 10^{8}$ & 4.53 & 1.38 & 4.59 & 1.60 \\
\hline West Virginia & 73 & 54 & $5.74 \times 10^{8}$ & 12.71 & 9.40 & 12.37 & 8.97 \\
\hline Wisconsin & 95 & 35 & $4.52 \times 10^{8}$ & 21.00 & 7.74 & 20.22 & 7.77 \\
\hline Wyoming & 33 & 17 & $1.10 \times 10^{9}$ & 3.01 & 1.55 & 4.01 & 2.91 \\
\hline Total U.S. & 2990 & 1279 & $4.47 \times 10^{10}$ & 6.68 & 2.86 & 7.34 & 3.09 \\
\hline
\end{tabular}

atales with no recorded accidents on any type of rail line were assigned the lowest observed state-level rate for that column. Thus, several states have identical (low) rates tor a given category. The national average fatality rate for railcar accident involvements averaged over 1985 and 1986 data is $1.26 \times 10^{-9}$ per railcar-kilometer. This value excludes fatalities due to rallroad "incidents" (on-track events in which the railroad property damage does not exceed the reporting threshold) because comparable truck "incidents" are not included in the OMC 50-T file. 
TABLE 2.5 Freight Vessel Accident and Casualty Involvement Fates on Domestic Waterways of the United States, Including Coastwise Shipping, 1985

\begin{tabular}{|c|c|c|c|c|c|}
\hline \multirow[b]{2}{*}{ Naterway System } & \multirow{2}{*}{$\begin{array}{l}\text { Total } \\
\text { Accident } \\
\text { Count }\end{array}$} & \multirow{2}{*}{$\begin{array}{l}\text { Total } \\
\text { Accident } \\
\text { Involve- } \\
\text { ments b } \\
\text { (no. of } \\
\text { vessels) }\end{array}$} & \multirow{2}{*}{$\begin{array}{l}\text { Total } \\
\text { Vessel } \\
\text { Casualty } \\
\text { Count }^{c}\end{array}$} & \multicolumn{2}{|c|}{$\begin{array}{c}\text { Accident and } \\
\text { Casualty Rates } \\
\left(10^{-6} \text { involvements per }\right. \\
\text { shipment-kilometer })\end{array}$} \\
\hline & & & & Accident & Casualty \\
\hline Atlantic Coast & 229 & 379 & 349 & 4.75 & 4.37 \\
\hline Culf Coast & 215 & 556 & 475 & 4.73 & 4.04 \\
\hline Pacific Coast & 68 & 115 & 2157 & 1.80 & 1.51 \\
\hline $\begin{array}{l}\text { Mississippi River } \\
\text { (including Ohio } \\
\text { Tennessee and }\end{array}$ & & & & & \\
\hline Missouri systems) & 412 & 3509 & 97 & 4.85 & 2.98 \\
\hline Great Lakes & 77 & 151 & 127 & 0.62 & 0.52 \\
\hline $\begin{array}{l}\text { Average for inland } \\
\text { waterways }\end{array}$ & 1001 & 4710 & 3205 & 3.83 & 2.79 \\
\hline Coastwise movements ${ }^{d}$ & 148 & 220 & 270 & 0.14 & 0.12 \\
\hline
\end{tabular}

á Estimated shipment weight for spent fuel cask on barge is 500 short tons (based on Tobin, Meshkov, and Jones 1.985).

${ }^{b}$ Count of vessels involved in accidents.

${ }^{c}$ Count of vessels sustaining reportable damage, fatality, or injury.

dComposite fatality rate for inland waterway movements is $2.32 \times 10^{-8}$ per shipment-kilometer; : =tality rate for all domestic water traffic (including coastwise) is $1.04 \times 10^{-8}$. Composite injury rate for inland waterway movements is $3.54 \times 10^{-8}$ per shipment-kilometer; injury rate for all domestic water traffic (including coastwise) is $1.39 \times 10^{-8}$. 


\section{AGRICULTURAL STATISTICS}

Food pathways were not considered in the transportation accident scenarios modeled in the EAs. The EA modeling used only a generic transfer factor for deriving the potential ingestion dose from an accident during the transport of SNF and HLW to any of the nominated geologic repository sites. No state-specific data were used to estimate the potential for food chain uptake. This approach ignored the variation in productive capability of states located in different areas of the country and thus ignored the potential for accident impacts (through the food chain) to be greater in some states than others.

In order to incorporate food chain uptakes into the transportation modeling, values for agricultural production for all states were calculeted. Agricultural production was divided into three main food groups: crops, meat (including eggs), and dairy. These three groups were selected because they correspond to the food groups considered in past studies relating to the food chain by the U.S. Nuclear Regulatory Commission. However, the U.S. Department of Agriculture (USDA) defines four major food groups: fruits and vegetables, cereals and grains, dairy and dairy products, and meat and meat alternatives. The first two of these USDA categories were combined to form the "crop" category considered in this report. The latter two USDA categories correspond directly to the dairy and meat categories used here. Production of each of the three main food groups is discussed in detail in Section 3.1 and Appendix A. Per capita consumption of these food groups is discussed in Section 3.2.

The 1982 Census of Agriculture (U.S. Bureau of the Census [USBC] 1984) was used as the primary source for all agricultural statistics, unless otherwise noted. The USDA Statistical Reporting Service was the secondary source of data. Although later statistics are available in some areas, the 1982 data were used for consistency across categories. These data represent a summary of agricultural statistics suitable for national-level modeling. If more-site-specific models are used, it is recommended that county-level agricultural statistics be combined with geographic information showing the location of agricultural land in each county.

\subsection{AGRICULTURAL PRODUCTION}

Production data by state for the agricultural products included in the crop, meat, and dairy categories were obtained from the USBC (1984) and USDA (1985a, 1985b, and $1985 \mathrm{c}$ ), and are listed in Appendix $A$. These data were input into a spreadsheet program for data manipulation, and the results are also listed in Appendix A. The formulas for manipulating the data are given in Appendix B, along with details (e.g., tables and page numbers) on the USBC and USDA data sources. Summary tables showing aggregate state production totals and production per unit area in the crop, meat, and dairy categories are presented and discussed in Sections 3.1.1-3.1.3. 


\subsubsection{Crops}

Crops are defined as corn, wheat, soybeans, potatoes, honey, sorghum, barley, oats, rye, sunflowers, dry beans, dry peas, sweet potatoes, sugar beets, peanuts, apples, cherries, grapes, nectarines, peaches, pears, plums and prunes, and other fruits. Crops produced specifically for animal feed, such as corn for silage, are not included. Although the census data are primarily reported in bushels, the spreadsheet program converted these data to pounds based on the fcllowing conversion factors (lb/bu): corn, sorghum, and rye, 56; wheat and soybeans, 60; barley, 48; oats, 32; and sweet potatoes, 55. These conversion factors were obtained from Jahnke (1987). Some crop data, e.g., fruits and nuts, are available in pounds and did not require conversion. The total weight of all crops produced in each state is presented in Tuble 3.1 .

For land area in farms, crop production in pounds per acre and kilograms per square kilometer is summarized in Table 3.2. This calculation was carried out by multiplying the percentage of land in farms (Table 3.1) by total land in the state (Table 3.1) and dividing production in each major category by the resulting "land in farms" numbe:. These values represent the average weight of crops produced per unit area in a given state. The rationale for using land in farms is that average production per acre is a more accurate representation of production on a given acre of agricultural land. The data presented in Table 3.3 represent production calculated using the total land area in the state. This results in a lower production number per unit area. Use of the data in Table 3.2 (land in farms) will result in more conservative estimates in modeling because agricultural production per unit area will be higher.

\subsubsection{Meat and Eggs}

Meat production includes cattle, calves, hogs, pigs, broilers, chickens, turkeys, and eggs. Data from the 1982 Census of Agriculture (U.S. Bureau of the Census 1984) were used for red meat, and data from the USDA (1985c) Statistical Bulletin 725 were used for poultry. These data represent the actual number of animals sold (with the exception of poultry). In the case of cattle/calves, only $55.13 \%$ of the animals sold were slaughtered, thus making their way into the food chain. This value was used to convert sales to slaughter for cattle/calves (ratio of animals sold to animals slaughtered in 1982 as renorted in Meatfacts (American Meat Institute 1986). The average weight of cattle/calves was obtained either from Meatfacts or by the following calculation:

$$
\text { weighted average }=\frac{(\text { cattle }<(b)+(\text { calves } \times 1 b)}{\text { cattle }+ \text { calves }}
$$

The total pounds produced in the state was calculated by:

total pounds $*$ state sales $\% \%$ slaughtered $\times$ weighted average 
TABLE 3.1 Summary of Total State Agricultural Production, $1982^{\mathrm{a}}$

\begin{tabular}{|c|c|c|c|c|c|}
\hline \multirow[b]{2}{*}{ State } & \multirow{2}{*}{$\begin{array}{c}\text { Land } \\
\text { in State } \\
\text { (acres) }\end{array}$} & \multirow{2}{*}{ in $\begin{array}{l}\text { Land } \\
(\%) \\
(\%)\end{array}$} & \multicolumn{3}{|c|}{ Total Production ( $1 \mathrm{~b}$ ) } \\
\hline & & & Crops & Meat & Dairy \\
\hline Al abama & $3.25 \times 10^{7}$ & 31.4 & $5.11 \times 10^{9}$ & $2.08 \times 10^{9}$ & $5.80 \times 10^{8}$ \\
\hline Alaska & $3.65 \times 10^{8}$ & 0.4 & $2.03 \times 10^{7}$ & $2.85 \times 10^{6}$ & $1.38 \times 10^{7}$ \\
\hline Arizona & $7.26 \times 10^{7}$ & 52.0 & $2.69 \times 10^{9}$ & $4.12 \times 10^{8}$ & $1.20 \times 10^{9}$ \\
\hline Arkansas & $3.33 \times 10^{7}$ & 44.1 & $1.06 \times 10^{10}$ & $2.91 \times 10^{9}$ & $8.28 \times 10^{8}$ \\
\hline California & $1.00 \times 10^{8}$ & 32.1 & $3.83 \times 10^{10}$ & $3.04 \times 10^{9}$ & $1.45 \times 10^{10}$ \\
\hline Colorado & $6.63 \times 10^{7}$ & 50.6 & $1.49 \times 10^{10}$ & $1.32 \times 10^{9}$ & $9.72 \times 10^{8}$ \\
\hline Connecticut & $3.12 \times 10^{6}$ & 14.2 & $1.21 \times 10^{8}$ & $1.66 \times 10^{8}$ & $6.44 \times 10^{8}$ \\
\hline Delaware & $1.24 \times 10^{6}$ & 53.0 & $1.60 \times 10^{9}$ & $5.49 \times 10^{8}$ & $1.37 \times 10^{8}$ \\
\hline Florida & $3.47 \times 10^{7}$ & 37.0 & $1.84 \times 10^{10}$ & $1.07 \times 10^{9}$ & $2.11 \times 10^{9}$ \\
\hline Georgia & $3.72 \times 10^{7}$ & 33.1 & $1.05 \times 10^{10}$ & $2.93 \times 10^{9}$ & $1.41 \times 10^{9}$ \\
\hline Hawai i & $4.11 \times 10^{6}$ & 47.6 & $1.24 \times 10^{8}$ & $7.89 \times 10^{7}$ & $1.44 \times 10^{8}$ \\
\hline Idaho & $5.27 \times 10^{7}$ & 26.4 & $2.53 \times 10^{10}$ & $5.72 \times 10^{8}$ & $2.25 \times 10^{9}$ \\
\hline Ill inois & $3.56 \times 10^{7}$ & 80.7 & $1.05 \times 10^{11}$ & $2.13 \times 10^{9}$ & $2.66 \times 10^{9}$ \\
\hline Indiana & $2.30 \times 10^{7}$ & 70.9 & $5.22 \times 10^{10}$ & $2.06 \times 10^{9}$ & $2.33 \times 10^{9}$ \\
\hline Iowa & $3.58 \times 10^{7}$ & 91.0 & $1.01 \times 10^{11}$ & $5.57 \times 10^{9}$ & $4.00 \times 10^{9}$ \\
\hline Kansas & $5.23 \times 10^{7}$ & 89.9 & $4.39 \times 10^{10}$ & $2.81 \times 10^{9}$ & $1.36 \times 10^{9}$ \\
\hline Kentucky & $2.54 \times 10^{7}$ & 55.8 & $1.14 \times 10^{10}$ & $7.11 \times 10^{8}$ & $2.36 \times 10^{9}$ \\
\hline Louisiana & $2.85 \times 10^{7}$ & 31.3 & $5.51 \times 10^{9}$ & $5.03 \times 10^{8}$ & $9.75 \times 10^{8}$ \\
\hline Maine & $1.98 \times 10^{7}$ & 7.4 & $2.67 \times 10^{9}$ & $2.86 \times 10^{8}$ & $7.27 \times 10^{8}$ \\
\hline Maryland & $6.30 \times 10^{6}$ & 40.6 & $4.93 \times 10^{9}$ & $8.96 \times 10^{8}$ & $1.58 \times 10^{9}$ \\
\hline Massachusetts & $5.01 \times 10^{6}$ & 12.2 & $2.03 \times 10^{8}$ & $7.01 \times 10^{7}$ & $6.02 \times 10^{8}$ \\
\hline Michigan & $3.65 \times 10^{7}$ & 30.0 & $2.55 \times 10^{10}$ & $7.29 \times 10^{8}$ & $5.25 \times 10^{9}$ \\
\hline Minnesota & $5.09 \times 10^{7}$ & 54.4 & $6.62 \times 10^{10}$ & $2.65 \times 10^{9}$ & $1.03 \times 10^{10}$ \\
\hline Mississippi & $3.02 \times 10^{7}$ & 41.1 & $7.28 \times 10^{9}$ & $1.25 \times 10^{9}$ & $9.00 \times 10^{8}$ \\
\hline Missouri & $4.41 \times 10^{7}$ & 66.3 & $2.66 \times 10^{10}$ & $2.19 \times 10^{9}$ & $2.91 \times 10^{9}$ \\
\hline Montana & $9.30 \times 10^{7}$ & 65.1 & $1.52 \times 10^{10}$ & $6.97 \times 10^{8}$ & $3.41 \times 10^{8}$ \\
\hline Nebraska & $4.91 \times 10^{7}$ & 91.7 & $5.71 \times 10^{10}$ & $3.29 \times 10^{9}$ & $1.34 \times 10^{9}$ \\
\hline Nevada & $7.03 \times 10^{7}$ & 14.2 & $6.48 \times 10^{8}$ & $9.92 \times 10^{7}$ & $2.25 \times 10^{8}$ \\
\hline
\end{tabular}


TABLI: 3.1 (Cont'd)

\begin{tabular}{|c|c|c|c|c|c|}
\hline \multirow[b]{2}{*}{ Slate } & $\begin{array}{c}\text { Land } \\
\text { in Stat } e^{a}\end{array}$ & \multirow{2}{*}{$\begin{array}{l}\text { Land } \\
\text { Farms } \\
(\%)\end{array}$} & \multicolumn{3}{|c|}{ Total Production ( $1 \mathrm{~b})$} \\
\hline & (acres) & & Crops & Meat & Dairy \\
\hline New hampshire & $5.76 \times 10^{6}$ & 8.2 & $5.34 \times 10^{7}$ & $3.41 \times 10^{7}$ & $3.55 \times 10^{8}$ \\
\hline New Jersey & $4.78 \times 10^{6}$ & 19.2 & $1.38 \times 10^{9}$ & $6.77 \times 10^{7}$ & $4.92 \times 10^{8}$ \\
\hline New Mexico & $7.77 \times 10^{7}$ & 60.6 & $1.80 \times 10^{9}$ & $4.58 \times 10^{8}$ & $8.12 \times 10^{8}$ \\
\hline New York & $3.03 \times 10^{7}$ & 30.3 & $7.36 \times 10^{9}$ & $5.59 \times 10^{8}$ & $1.11 \times 10^{10}$ \\
\hline North Carolina & $3.13 \times 10^{7}$ & 33.0 & $1.30 \times 10^{10}$ & $2.65 \times 10^{9}$ & $1.69 \times 10^{9}$ \\
\hline North Dakuta & $4.44 \times 10^{7}$ & 90.7 & $3.65 \times 10^{10}$ & $4.52 \times 10^{8}$ & $1.02 \times 10^{9}$ \\
\hline Ohio & $2.62 \times 10^{7}$ & 58.7 & $3.48 \times 10^{10}$ & $1.30 \times 10^{9}$ & $4.55 \times 10^{9}$ \\
\hline Oklahoma & $4.39 \times 10^{7}$ & 73.7 & $1.22 \times 10^{10}$ & $1.52 \times 10^{9}$ & $1.17 \times 10^{9}$ \\
\hline Oregon & $6.16 \times 10^{7}$ & 28.8 & $8.05 \times 10^{9}$ & $5.04 \times 10^{8}$ & $1.30 \times 10^{9}$ \\
\hline Pennsyl vania & $2.87 \times 10^{7}$ & 28.9 & $9.27 \times 10^{9}$ & $1.57 \times 10^{9}$ & $9.26 \times 10^{9}$ \\
\hline Khode 1 sland & $6.75 \times 10^{5}$ & 9.3 & $7.72 \times 10^{7}$ & $1.24 \times 10^{7}$ & $4.60 \times 10^{7}$ \\
\hline South Carolina & $1.93 \times 10^{7}$ & 28.9 & $4.79 \times 10^{9}$ & $5.55 \times 10^{8}$ & $5.67 \times 10^{8}$ \\
\hline South Dakota & $4.86 \times 10^{7}$ & 90.1 & $2.18 \times 10^{10}$ & $1.39 \times 10^{9}$ & $1.76 \times 10^{9}$ \\
\hline 'lennessee & $2.63 \times 10^{7}$ & 47.4 & $7.86 \times 10^{9}$ & $8.75 \times 10^{8}$ & $2.33 \times 10^{9}$ \\
\hline Texas & $1.68 \times 10^{8}$ & 78.3 & $3.27 \times 10^{10}$ & $4.88 \times 10^{9}$ & $3.78 \times 10^{9}$ \\
\hline Utah & $5.25 \times 10^{7}$ & 18.6 & $1.24 \times 10^{9}$ & $2.68 \times 10^{8}$ & $1.16 \times 10^{9}$ \\
\hline Vermont & $5.93 \times 10^{6}$ & 20.5 & $1.32 \times 10^{8}$ & $6.71 \times 10^{7}$ & $2.39 \times 10^{9}$ \\
\hline Virginia & $2.54 \times 10^{7}$ & 37.1 & $6.23 \times 10^{9}$ & $1.05 \times 10^{9}$ & $2.06 \times 10^{9}$ \\
\hline Washington & $4.26 \times 10^{7}$ & 38.7 & $1.95 \times 10^{10}$ & $6.50 \times 10^{8}$ & $3.22 \times 10^{9}$ \\
\hline West virginia & $1.54 \times 10^{7}$ & 23.1 & $7.45 \times 10^{8}$ & $2.23 \times 10^{8}$ & $3.49 \times 10^{8}$ \\
\hline Wisconsin & $3.48 \times 10^{7}$ & 49.5 & $2.34 \times 10^{10}$ & $1.31 \times 10^{9}$ & $2.32 \times 10^{10}$ \\
\hline Wyoming & $6.21 \times 10^{7}$ & 54.0 & $3.20 \times 10^{9}$ & $3.54 \times 13^{8}$ & $1.37 \times 10^{8}$ \\
\hline U.S. Total & $2.27 \times 10^{9}$ & 43.6 & $1.13 \times 10^{12}$ & $6.20 \times 10^{10}$ & $1.36 \times 10^{11}$ \\
\hline
\end{tabular}

a Sources given in Appendix B; crops, meal, and dairy defined in Section 3.1. 
TABLE 3.2 Summary of State Agricultural Production for Land in Farms, 1982

\begin{tabular}{|c|c|c|c|c|c|c|}
\hline \multirow[b]{2}{*}{ State } & \multicolumn{2}{|c|}{ Crops } & \multicolumn{2}{|c|}{ Meat } & \multicolumn{2}{|c|}{ Dairy } \\
\hline & $1 \mathrm{~b} / \mathrm{acre}$ & $\mathrm{kg} / \mathrm{km}^{2}$ & $1 \mathrm{~b} / \mathrm{acre}$ & $\mathrm{kg} / \mathrm{km}^{2}$ & $1 \mathrm{~b} / \mathrm{acre}$ & $\mathrm{kg} / \mathrm{km}^{2}$ \\
\hline Al abama & $5.00 \times 10^{2}$ & $1.76 \times 10^{4}$ & $2.04 \times 10^{2}$ & $7.19 \times 10^{3}$ & $5.69 \times 10^{1}$ & $2.00 \times 10^{3}$ \\
\hline Alaska & $1.39 \times 10^{1}$ & $6.22 \times 10^{0}$ & $1.95 \times 10^{0}$ & $8.73 \times 10^{-1}$ & $9.44 \times 10^{0}$ & $4.23 \times 10^{0}$ \\
\hline Arizona & $7.13 \times 10^{1}$ & $4.15 \times 10^{3}$ & $1.09 \times 10^{1}$ & $6.36 \times 10^{2}$ & $3.18 \times 10^{1}$ & $1.85 \times 10^{3}$ \\
\hline Arkansas & $7.24 \times 10^{2}$ & $3.58 \times 10^{4}$ & $1.98 \times 10^{2}$ & $9.77 \times 10^{3}$ & $5.63 \times 10^{1}$ & $2.78 \times 10^{3}$ \\
\hline Cal ifornia & $1.19 \times 10^{3}$ & $4.29 \times 10^{4}$ & $9.46 \times 10^{1}$ & $3.41 \times 10^{3}$ & $4.52 \times 10^{2}$ & $1.63 \times 10^{4}$ \\
\hline Colorado & $4.44 \times 10^{2}$ & $2.52 \times 10^{4}$ & $3.94 \times 10^{1}$ & $2.24 \times 10^{3}$ & $2.90 \times 10^{1}$ & $1.64 \times 10^{3}$ \\
\hline Connecticut & $2.74 \times 10^{2}$ & $4.36 \times 10^{3}$ & $3.75 \times 10^{2}$ & $5.97 \times 10^{3}$ & $1.45 \times 10^{3}$ & $2.32 \times 10^{4}$ \\
\hline Delaware & $2.45 \times 10^{3}$ & $1.45 \times 10^{5}$ & $8.38 \times 10^{2}$ & $4.98 \times 10^{4}$ & $2.09 \times 10^{2}$ & $1.24 \times 10^{4}$ \\
\hline Florida & $1.43 \times 10^{3}$ & $5.94 \times 10^{4}$ & $8.37 \times 10^{1}$ & $3.47 \times 10^{3}$ & $1.64 \times 10^{2}$ & $6.82 \times 10^{3}$ \\
\hline Ceorgia & $8.50 \times 10^{2}$ & $3.15 \times 10^{4}$ & $2.38 \times 10^{2}$ & $8.84 \times 10^{3}$ & $1.15 \times 10^{2}$ & $4.26 \times 10^{3}$ \\
\hline Hawai i & $6.33 \times 10^{1}$ & $3.38 \times 10^{3}$ & $4.03 \times 10^{1}$ & $2.15 \times 10^{3}$ & $7.36 \times 10^{1}$ & $3.93 \times 10^{3}$ \\
\hline Idano & $1.81 \times 10^{3}$ & $5.37 \times 10^{4}$ & $4.11 \times 10^{1}$ & $1.22 \times 10^{3}$ & $1.62 \times 10^{2}$ & $4.79 \times 10^{3}$ \\
\hline Illinois & $3.64 \times 10^{3}$ & $3.29 \times 10^{5}$ & $7.41 \times 10^{1}$ & $6.70 \times 10^{3}$ & $9.25 \times 10^{1}$ & $8.36 \times 10^{3}$ \\
\hline Indiana & $3.20 \times 10^{3}$ & $2.54 \times 10^{5}$ & $1.26 \times 10^{2}$ & $1.00 \times 10^{4}$ & $1.43 \times 10^{2}$ & $1.14 \times 10^{4}$ \\
\hline Iowa & $3.11 \times 10^{3}$ & $3.17 \times 10^{5}$ & $1.71 \times 10^{2}$ & $1.74 \times 10^{4}$ & $1.23 \times 10^{2}$ & $1.25 \times 10^{4}$ \\
\hline Kansas & $9.32 \times 10^{2}$ & $9.40 \times 10^{4}$ & $5.96 \times 10^{1}$ & $6.01 \times 10^{3}$ & $2.88 \times 10^{1}$ & $2.90 \times 10^{3}$ \\
\hline Kentucky & $8.07 \times 10^{2}$ & $5.05 \times 10^{4}$ & $5.02 \times 10^{1}$ & $3.14 \times 10^{3}$ & $1.67 \times 10^{2}$ & $\therefore 04 \cdot 10^{4}$ \\
\hline Louisiana & $6.18 \times 10^{2}$ & $2.17 \times 10^{4}$ & $5.64 \times 10^{1}$ & $1.98 \times 10^{3}$ & $1.09 \times 10^{2}$ & $3.84 \times 10^{3}$ \\
\hline Maine & $1.82 \times 10^{3}$ & $1.51 \times 10^{4}$ & $1.95 \times 10^{2}$ & $1.62 \times 10^{3}$ & $4.95 \times 10^{2}$ & $4.11 \times 10^{3}$ \\
\hline Maryl and & $1.93 \times 10^{3}$ & $8.79 \times 10^{4}$ & $3.50 \times 10^{2}$ & $1.59 \times 10^{4}$ & $6.19 \times 10^{2}$ & $2.81 \times 10^{4}$ \\
\hline Massachusetts & $3.32 \times 10^{2}$ & $4.55 \times 10^{3}$ & $1.15 \times 10^{2}$ & $1.57 \times 10^{3}$ & $9.85 \times 10^{2}$ & $1.35 \times 10^{4}$ \\
\hline Michigan & $2.33 \times 10^{3}$ & $7.84 \times 10^{4}$ & $6.66 \times 10^{1}$ & $2.24 \times 10^{3}$ & $4.80 \times 10^{2}$ & $1.62 \times 10^{4}$ \\
\hline Minnesota & $2.39 \times 10^{3}$ & $1.46 \times 10^{5}$ & $9.56 \times 10^{1}$ & $5.83 \times 10^{3}$ & $3.73 \times 10^{2}$ & $2.28 \times 10^{2}$ \\
\hline Mississippi & $5.86 \times 10^{2}$ & $2.70 \times 10^{4}$ & $1.01 \times 10^{2}$ & $4.65 \times 10^{3}$ & $7.24 \times 10^{1}$ & $3.34 \times 10^{3}$ \\
\hline Missouri & $9.09 \times 10^{2}$ & $6.76 \times 10^{4}$ & $7.48 \times 10^{1}$ & $5.56 \times 10^{3}$ & $9.93 \times 10^{1}$ & $7.38 \times 10^{3}$ \\
\hline Montana & $2.52 \times 10^{2}$ & $1.84 \times 10^{4}$ & $1.15 \times 10^{1}$ & $8.39 \times 10^{2}$ & $5.63 \times 10^{0}$ & $4.11 \times i 0^{2}$ \\
\hline Nebraska & $1.27 \times 10^{3}$ & $1.31 \times 10^{5}$ & $7.31 \times 10^{1}$ & $7.51 \times 10^{3}$ & $2.98 \times 10^{1}$ & $3.06 \times 10^{3}$ \\
\hline Nevada & $6.49 \times 10^{1}$ & $1.03 \times 10^{3}$ & $9.93 \times 10^{0}$ & $1.58 \times 10^{2}$ & $2.25 \times 10^{1}$ & $3.59 \times 10^{2}$ \\
\hline
\end{tabular}




\begin{tabular}{|c|c|c|c|c|c|c|}
\hline \multirow[b]{2}{*}{ Stale } & \multicolumn{2}{|c|}{ Crops } & \multicolumn{2}{|c|}{ Meat } & \multicolumn{2}{|c|}{ Dairy } \\
\hline & lb/acre & $\mathrm{kg} / \mathrm{km}^{2}$ & lb/acre & $\mathrm{kg} / \mathrm{km}^{2}$ & $\mathrm{lb} / \mathrm{acre}$ & $\mathrm{kg} / \mathrm{km}^{2}$ \\
\hline New Hampshire & $1.13 \times 10^{2}$ & $1.04 \times 10^{3}$ & $7.22 \times 10^{1}$ & $6.63 \times 10^{2}$ & $7.73 \times 10^{2}$ & $7.11 \times 10^{3}$ \\
\hline New Jersey & $1.50 \times 10^{3}$ & $3.23 \times 10^{4}$ & $7.38 \times 10^{1}$ & $1.59 \times 10^{3}$ & $5.36 \times 10^{2}$ & $1.15 \times 10^{4}$ \\
\hline New Mexico & $3.83 \times 10^{1}$ & $2.60 \times 10^{3}$ & $9.72 \times 10^{0}$ & $6.61 \times 10^{2}$ & $1.73 \times 10^{1}$ & $1.17 \times 10^{3}$ \\
\hline New York & $8.01 \times 10^{2}$ & $2.72 \times 10^{4}$ & $6.08 \times 10^{1}$ & $2.07 \times 10^{3}$ & $1.21 \times 10^{3}$ & $4.10 \times 10^{4}$ \\
\hline North Carolina & $1.26 \times 10^{3}$ & $4.65 \times 10^{4}$ & $2.57 \times 10^{2}$ & $9.51 \times 10^{3}$ & $1.63 \times 10^{2}$ & $6.05 \times 10^{3}$ \\
\hline North Dakota & $9.08 \times 10^{2}$ & $9.23 \times 10^{4}$ & $1.12 \times 10^{1}$ & $1.14 \times 10^{3}$ & $2.54 \times 10^{1}$ & $2.58 \times 10^{3}$ \\
\hline Ohio & $2.26 \times 10^{3}$ & $1.49 \times 10^{5}$ & $8.44 \times 10^{1}$ & $5.55 \times 10^{3}$ & $2.95 \times 10^{2}$ & $1.94 \times 10^{4}$ \\
\hline Okl ahoma & $3.76 \times 10^{2}$ & $3.10 \times 10^{4}$ & $4.69 \times 10^{1}$ & $3.87 \times 10^{3}$ & $3.60 \times 10^{1}$ & $2.97 \times 10^{3}$ \\
\hline Oregon & $4.54 \times 10^{2}$ & $1.47 \times 10^{4}$ & $2.84 \times 10^{1}$ & $9.18 \times 10^{\circ}$ & $7.34 \times 10^{1}$ & $2.37 \times 10^{3}$ \\
\hline Pennsylvania & $1.12 \times 10^{3}$ & $3.62 \times 10^{4}$ & $1.89 \times 10^{2}$ & $6.13 \times 10^{3}$ & $1.12 \times 10^{3}$ & $3.61 \times 10^{4}$ \\
\hline Rhode Is I and & $1.23 \times 10^{3}$ & $1.28 \times 10^{4}$ & $1.98 \times 10^{2}$ & $2.06 \times 10^{3}$ & $7.33 \times 10^{2}$ & $7.64 \times 10^{3}$ \\
\hline South Carolina & $8.57 \times 10^{2}$ & $2.78 \times 10^{4}$ & $9.94 \times 10^{1}$ & $3.22 \times 10^{3}$ & $1.01 \times 10^{2}$ & $3.29 \times 10^{3}$ \\
\hline South Dakota & $4.99 \times 10^{2}$ & $5.04 \times 10^{4}$ & $3.17 \times 10^{1}$ & $3.20 \times 10^{3}$ & $4.02 \times 10^{1}$ & $4.06 \times 10^{3}$ \\
\hline Tennessee & $6.30 \times 10^{2}$ & $3.34 \times 10^{4}$ & $7.01 \times 10^{1}$ & $3.72 \times 10^{3}$ & $1.86 \times 10^{2}$ & $9.90 \times 10^{3}$ \\
\hline Texas & $2.49 \times 10^{2}$ & $2.19 \times 10^{4}$ & $3.71 \times 10^{1}$ & $3.26 \times 10^{3}$ & $2.88 \times 10^{1}$ & $2.53 \times 10^{3}$ \\
\hline Utah & $1.27 \times 10^{2}$ & $2.66 \times 10^{3}$ & $2.75 \times 10^{1}$ & $5.72 \times 10^{2}$ & $1.19 \times 10^{2}$ & $2.48 \times 10^{3}$ \\
\hline Vermont & $8.36 \times 10^{1}$ & $2.48 \times 10^{3}$ & $4.27 \times 10^{1}$ & $1.27 \times 10^{3}$ & $1.52 \times 10^{3}$ & $4.50 \times 10^{4}$ \\
\hline Virginia & $6.61 \times 10^{2}$ & $2.75 \times 10^{4}$ & $1.11 \times 10^{2}$ & $4.61 \times 10^{3}$ & $2.18 \times 10^{2}$ & $9.08 \times 10^{3}$ \\
\hline Washington & $1.18 \times 10^{3}$ & $5.13 \times 10^{4}$ & $3.94 \times 10^{1}$ & $1.71 \times 10^{3}$ & $1.96 \times 10^{2}$ & $8.48 \times 10^{3}$ \\
\hline West Virginia & $2.09 \times 10^{2}$ & $5.41 \times 10^{3}$ & $6.26 \times 10^{1}$ & $1.62 \times 10^{3}$ & $9.79 \times 10^{1}$ & $2.53 \times 10^{3}$ \\
\hline Wisconsin & $1.36 \times 10^{3}$ & $7.53 \times 10^{4}$ & $7.57 \times 10^{1}$ & $4.20 \times 10^{3}$ & $1.35 \times 10^{3}$ & $7.47 \times 10^{4}$ \\
\hline Wyoming & $9.54 \times 10^{1}$ & $5.77 \times 10^{3}$ & $1.06 \times 10^{1}$ & $6.39 \times 10^{2}$ & $4.09 \times 10^{0}$ & $2.47 \times 10^{2}$ \\
\hline U.S. Total & $1.14 \times 10^{3}$ & $5.58 \times 10^{4}$ & $6.28 \times 10^{1}$ & $3.07 \times 10^{3}$ & $1.37 \times 10^{2}$ & $6.71 \times 10^{3}$ \\
\hline
\end{tabular}

${ }^{a}$ Sources given in Appendix B; crops, meat, and dairy defined in Sec. 3.1 . 
'TAHLL 3.3 Summary of State Agricultural Production for Total Land in State, 1982

\begin{tabular}{|c|c|c|c|c|c|c|}
\hline \multirow[b]{2}{*}{ State } & \multicolumn{2}{|c|}{ Crops } & \multicolumn{2}{|c|}{ Meat } & \multicolumn{2}{|c|}{ Dairy } \\
\hline & lb/acre & $\mathrm{kg} / \mathrm{km}^{2}$ & $1 \mathrm{~b} / \mathrm{acre}$ & $\mathrm{kg} / \mathrm{km}^{2}$ & lb/acre & $\mathrm{kg} / \mathrm{km}^{2}$ \\
\hline Al abama & $1.57 \times 10^{2}$ & $5.53 \times 10^{3}$ & $6.41 \times 10^{1}$ & $2.26 \times 10^{3}$ & $1.79 \times 10^{1}$ & $6.28 \times 10^{2}$ \\
\hline Alaska & $5.55 \times 10^{-2}$ & $2.49 \times 10^{-2}$ & $7.79 \times 10^{-3}$ & $3.49 \times 10^{-3}$ & $3.78 \times 10^{-2}$ & $1.69 \times 10^{-2}$ \\
\hline Arizona & $3.71 \times 10^{1}$ & $2.16 \times 10^{3}$ & $5.68 \times 10^{0}$ & $3.31 \times 10^{2}$ & $1.65 \times 10^{1}$ & $9.64 \times 10^{2}$ \\
\hline Arkansas & $3.19 \times 10^{2}$ & $1.58 \times 10^{4}$ & $8.72 \times 10^{1}$ & $4.31 \times 10^{3}$ & $2.48 \times 10^{1}$ & $1.23 \times 10^{3}$ \\
\hline Cal ifornia & $3.83 \times 10^{2}$ & $1.38 \times 10^{4}$ & $3.04 \times 10^{1}$ & $1.09 \times 10^{3}$ & $1.45 \times 10^{2}$ & $5.23 \times 10^{3}$ \\
\hline Colorado & $2.25 \times 10^{2}$ & $1.27 \times 10^{4}$ & $2.00 \times 10^{1}$ & $1.33 \times 10^{3}$ & $1.47 \times 10^{1}$ & $8.31 \times 10^{2}$ \\
\hline Connecticut & $3.89 \times 10^{1}$ & $6.19 \times 10^{2}$ & $5.33 \times 10^{1}$ & $8.48 \times 10^{2}$ & $2.07 \times 10^{2}$ & $3.29 \times 10^{3}$ \\
\hline Del aware & $1.30 \times 10^{3}$ & $7.70 \times 10^{4}$ & $4.44 \times 10^{2}$ & $2.64 \times 10^{4}$ & $1.11 \times 10^{2}$ & $6.58 \times 10^{3}$ \\
\hline Florida & $5.30 \times 10^{2}$ & $2.20 \times 10^{4}$ & $3.10 \times 10^{1}$ & $1.28 \times 10^{3}$ & $6.09 \times 10^{1}$ & $2.52 \times 10^{3}$ \\
\hline Georgia & $2.81 \times 10^{2}$ & $1.04 \times 10^{4}$ & $7.88 \times 10^{1}$ & $2.93 \times 10^{3}$ & $3.80 \times 10^{1}$ & $1.41 \times 10^{3}$ \\
\hline Hawai i & $3.01 \times 10^{1}$ & $1.61 \times 10^{3}$ & $1.92 \times 10^{1}$ & $1.02 \times 10^{3}$ & $3.50 \times 10^{1}$ & $1.87 \times 10^{3}$ \\
\hline Idaho & $4.79 \times 10^{2}$ & $1.42 \times 10^{4}$ & $1.08 \times 10^{1}$ & $3.21 \times 10^{2}$ & $4.27 \times 10^{1}$ & $1.26 \times 10^{3}$ \\
\hline Illinois & $2.94 \times 10^{3}$ & $2.66 \times 10^{5}$ & $5.98 \times 10^{1}$ & $5.41 \times 10^{3}$ & $7.46 \times 10^{1}$ & $6.75 \times 10^{3}$ \\
\hline Indiana & $2.27 \times 10^{3}$ & $1.80 \times 10^{5}$ & $8.96 \times 10^{1}$ & $7.12 \times 10^{3}$ & $1.01 \times 10^{2}$ & $8.07 \times 10^{3}$ \\
\hline Iowa & $2.83 \times 10^{3}$ & $2.89 \times 10^{5}$ & $1.56 \times 10^{2}$ & $1.59 \times 10^{4}$ & $1.12 \times 10^{2}$ & $1.14 \times 10^{4}$ \\
\hline Kansas & $8.38 \times 10^{2}$ & $8.45 \times 10^{4}$ & $5.36 \times 10^{1}$ & $5.40 \times 10^{3}$ & $2.59 \times 10^{1}$ & $2.61 \times 10^{3}$ \\
\hline Kentucky & $4.50 \times 10^{2}$ & $2.82 \times 10^{4}$ & $2.80 \times 10^{1}$ & $1.75 \times 10^{3}$ & $9.31 \times 10^{1}$ & $5.82 \times 10^{3}$ \\
\hline Louisiana & $1.93 \times 10^{2}$ & $6.79 \times 10^{3}$ & $1.77 \times 10^{1}$ & $6.19 \times 10^{2}$ & $3.42 \times 10^{1}$ & $1.20 \times 10^{3}$ \\
\hline Maine & $1.35 \times 10^{2}$ & $1.12 \times 10^{3}$ & $1.44 \times 10^{1}$ & $1.20 \times 10^{2}$ & $3.66 \times 10^{1}$ & $3.04 \times 10^{2}$ \\
\hline Maryland & $7.84 \times 10^{2}$ & $3.57 \times 10^{4}$ & $1.42 \times 10^{2}$ & $6.48 \times 10^{3}$ & $2.51 \times 10^{2}$ & $1.14 \times 10^{4}$ \\
\hline Massachusetts & $4.06 \times 10^{1}$ & $5.55 \times 10^{2}$ & $1.40 \times 10^{1}$ & $1.91 \times 10^{2}$ & $1.20 \times 10^{2}$ & $1.64 \times 10^{3}$ \\
\hline Michigan & $6.99 \times 10^{2}$ & $2.35 \times 10^{4}$ & $2.00 \times 10^{1}$ & $6.72 \times 10^{2}$ & $1.44 \times 10^{2}$ & $4.85 \times 10^{3}$ \\
\hline Minnesota & $1.30 \times 10^{3}$ & $7.93 \times 10^{4}$ & $5.20 \times 10^{1}$ & $3.17 \times 10^{3}$ & $2.03 \times 10^{2}$ & $1.24 \times 10^{4}$ \\
\hline Mississippi & $2.41 \times 10^{2}$ & $1.11 \times 10^{4}$ & $4.15 \times 10^{1}$ & $1.91 \times 10^{3}$ & $2.98 \times 10^{1}$ & $1.37 \times 10^{3}$ \\
\hline Missouri & $6.03 \times 10^{2}$ & $4.48 \times 10^{4}$ & $4.96 \times 10^{1}$ & $3.68 \times 10^{3}$ & $6.58 \times 10^{1}$ & $4.89 \times 10^{3}$ \\
\hline Montana & $1.64 \times 10^{2}$ & $1.20 \times 10^{4}$ & $7.49 \times 10^{0}$ & $5.46 \times 10^{2}$ & $3.66 \times 10^{0}$ & $2.67 \times 10^{2}$ \\
\hline Nebraska & $1.16 \times 10^{3}$ & $1.20 \times 10^{5}$ & $6.70 \times 10^{1}$ & $6.89 \times 10^{3}$ & $2.73 \times 10^{1}$ & $2.81 \times 10^{3}$ \\
\hline Nevada & $9.22 \times 10^{0}$ & $1.47 \times 10^{2}$ & $1.41 \times 10^{0}$ & $2.25 \times 10^{1}$ & $3.20 \times 10^{0}$ & $5.09 \times 10^{1}$ \\
\hline
\end{tabular}




\begin{tabular}{|c|c|c|c|c|c|c|}
\hline \multirow[b]{2}{*}{ State } & \multicolumn{2}{|c|}{ Crops } & \multicolumn{2}{|c|}{ Meat } & \multicolumn{2}{|c|}{ Dairy } \\
\hline & lb/acre & $\mathrm{kg} / \mathrm{km}^{2}$ & $1 \mathrm{~b} / \mathrm{acre}$ & $\mathrm{kg} / \mathrm{km}^{2}$ & $1 \mathrm{~b} / \mathrm{acre}$ & $\mathrm{kg} / \mathrm{km}^{2}$ \\
\hline New Hampshire & $9.29 \times 10^{0}$ & $8.53 \times 10^{1}$ & $5.92 \times 10^{0}$ & $5.44 \times 10^{1}$ & $6.34 \times 10^{1}$ & $5.83 \times 10^{2}$ \\
\hline New Jersey & $2.88 \times 10^{2}$ & $6.21 \times 10^{3}$ & $1.42 \times 10^{1}$ & $3.05 \times 10^{2}$ & $1.03 \times 10^{2}$ & $2.22 \times 10^{3}$ \\
\hline New Mexico & $2.32 \times 10^{1}$ & $1.58 \times 10^{3}$ & $5.89 \times 10^{0}$ & $4.00 \times 10^{2}$ & $1.05 \times 10^{1}$ & $7.10 \times 10^{2}$ \\
\hline New York & $2.43 \times 10^{2}$ & $8.24 \times 10^{3}$ & $1.84 \times 10^{1}$ & $6.26 \times 10^{2}$ & $3.66 \times 10^{2}$ & $1.24 \times 10^{4}$ \\
\hline North Carolina & $4.14 \times 10^{2}$ & $1.53 \times 10^{4}$ & $8.48 \times 10^{1}$ & $3.14 \times 10^{3}$ & $5.39 \times 10^{1}$ & $1.99 \times 10^{3}$ \\
\hline North Dakota & $8.23 \times 10^{2}$ & $8.37 \times 10^{4}$ & $1.02 \times 10^{1}$ & $1.04 \times 10^{3}$ & $2.30 \times 10^{1}$ & $2.34 \times 10^{3}$ \\
\hline Ohio & $1.33 \times 10^{3}$ & $8.72 \times 10^{4}$ & $4.95 \times 10^{1}$ & $3.26 \times 10^{3}$ & $1.73 \times 10^{2}$ & $1.14 \times 10^{4}$ \\
\hline Oklahoma & $2.77 \times 10^{2}$ & $2.29 \times 10^{4}$ & $3.45 \times 10^{1}$ & $2.85 \times 10^{3}$ & $2.65 \times 10^{1}$ & $2.19 \times 10^{3}$ \\
\hline Oregon & $1.31 \times 10^{2}$ & $4.22 \times 10^{3}$ & $8.19 \times 10^{0}$ & $2.64 \times 10^{2}$ & $2.11 \times 10^{1}$ & $6.82 \times 10^{2}$ \\
\hline Pennsylvania & $3.23 \times 10^{2}$ & $1.04 \times 10^{4}$ & $5.47 \times 10^{1}$ & $1.77 \times 10^{3}$ & $3.22 \times 10^{2}$ & $1.04 \times 10^{4}$ \\
\hline Rhode Island & $1.14 \times 10^{2}$ & $1.19 \times 10^{3}$ & $1.84 \times 10^{1}$ & $1.92 \times 10^{2}$ & $6.81 \times 10^{1}$ & $7.10 \times 10^{2}$ \\
\hline South Carolina & $2.48 \times 10^{2}$ & $8.03 \times 10^{3}$ & $2.87 \times 10^{1}$ & $9.30 \times 10^{2}$ & $2.93 \times 10^{1}$ & $9.50 \times 10^{2}$ \\
\hline South Dakota & $4.49 \times 10^{2}$ & $4.54 \times 10^{4}$ & $2.86 \times 10^{1}$ & $2.89 \times 10^{3}$ & $3.62 \times 10^{1}$ & $3.66 \times 10^{3}$ \\
\hline Tennessee & $2.98 \times 10^{2}$ & $1.59 \times 10^{4}$ & $3.32 \times 10^{1}$ & $1.76 \times 10^{3}$ & $8.83 \times 10^{1}$ & $4.69 \times 10^{3}$ \\
\hline Texas & $1.95 \times 10^{2}$ & $1.71 \times 10^{4}$ & $2.91 \times 10^{1}$ & $2.55 \times 10^{3}$ & $2.25 \times 10^{1}$ & $1.98 \times 10^{3}$ \\
\hline Utah & $2.37 \times 10^{1}$ & $4.94 \times 10^{2}$ & $5.11 \times 10^{0}$ & $1.06 \times 10^{2}$ & $2.21 \times 10^{1}$ & $4.61 \times 10^{2}$ \\
\hline Vermont & $2.22 \times 10^{1}$ & $6.58 \times 10^{2}$ & $1.13 \times 10^{1}$ & $3.36 \times 10^{2}$ & $4.02 \times 10^{2}$ & $1.19 \times 10^{4}$ \\
\hline Virginia & $2.45 \times 10^{2}$ & $1.02 \times 10^{4}$ & $4.11 \times 10^{1}$ & $1.71 \times 10^{3}$ & $8.10 \times 10^{1}$ & $3.37 \times 10^{3}$ \\
\hline Washington & $4.58 \times 10^{2}$ & $1.99 \times 10^{4}$ & $1.53 \times 10^{1}$ & $6.62 \times 10^{2}$ & $7.57 \times 10^{1}$ & $3.28 \times 10^{3}$ \\
\hline West Virginia & $4.83 \times 10^{1}$ & $1.25 \times 10^{3}$ & $1.44 \times 10^{1}$ & $3.74 \times 10^{2}$ & $2.26 \times 10^{1}$ & $5.85 \times 10^{2}$ \\
\hline Wisconsin & $6.72 \times 10^{2}$ & $3.73 \times 10^{4}$ & $3.75 \times 10^{1}$ & $2.08 \times 10^{3}$ & $6.67 \times 10^{2}$ & $3.70 \times 10^{4}$ \\
\hline Wyoming & $5.15 \times 10^{1}$ & $3.12 \times 10^{3}$ & $5.70 \times 10^{0}$ & $3.45 \times 10^{2}$ & $2.21 \times 10^{0}$ & $1.34 \times 10^{2}$ \\
\hline U.S. Total & $4.97 \times 10^{2}$ & $2.43 \times 10^{4}$ & $2.74 \times 10^{1}$ & $1.34 \times 10^{3}$ & $5.98 \times 10^{1}$ & $2.92 \times 10^{3}$ \\
\hline
\end{tabular}

${ }^{a}$ Sources given in Appendix B; crops, meat, and dairy defined in Sec. 3.1 . 
Hogs/pigs data were calculated similarly, but using $87.4 \%$ of sales as slaughtered and without taking the weighted mean.

Chicken data, reported in thousands of pounds, were directly input from Statistical Bulletin 725 (USDA 1985c). The pounds sold was added to on-farm constimption to obtain total production. These data were multiplied by a conversion factor of $61.77 \%$ to offset those chickens that are sold but not used for human consumption (percent consumed calculated from USDA [1983]). Broiler production and turkey production were entered directly from the 1982 Census of Agriculture in numbers of head sold; these data were then converted by the percent consumed -- $73.15 \%$ and $79.70 \%$, respectively -- and by a conversion factor based on average weights of $4.03 \mathrm{lb}$ and $19.19 \mathrm{lb}$, respectively (conversion provided by Sitzman [1987]).

Egg prodiction was calculated by taking total production from Statistical Bulletin 721 (USDA 1985a), dividing total production by 12 to produce doze.s, and multiplying by a conversion factor of $1.57 \mathrm{lb} / \mathrm{doz}$ (the conversion factor provided by the USDA National Agricultural Statistical Service).

For each state, meat production per acre was calculated by summing cattle/calves, hogs/pigs, broilers, chickens, and turkeys and dividing by the total land in farms (acres) in that state. The resulting data (Table 3.2) represent average meat production per unit area of farmland in a given state (lb/acre or $\mathrm{kg} / \mathrm{km}^{2}$ ). Total production divided by the total area in the state is presented in Table 3.3. Meat production does not include fish.

\subsubsection{Dairy}

Dairy production was calculated using milk production for 1982 (in millions of pounds) as reported in Statistical Bulletin 722 (USDA 1985b). These data are presented in Table 3.1. Dairy production data for land in farms and land in state are presented in Tables 3.2 and 3.3, respectively. These data were calculated in the same manner as described in Section 3.1.2 for meat.

\subsection{PER CAPITA CONSUMPTION}

Estimated per capita food consumption data were obtained from the USDA (1986). These data were aggregated into the three major food groups (crops, meat, dairy) and are presented in Table 3.4. Slight differences exist in the definitions of crops, meat, and dairy used in Table 3.4 compared with the definitions used in Tables 3.1-3.3. In acdition, the definitions of agricultural production are not directly comparable. In Table 3.4, agricultural production is defined as imports and beginning-of-year inventories minus exports, military use, year-end inventories, and nonfood use; in Tables 3.1-3.3, agricultural production represents production in the United States for the year 1982. Also, Table 3.4 is consumption-based, whereas Tables 3.1-3.3 are production-ibased.

It is recommended that the most recent consumpticu estimates be used for modeling purposes, although there are some duviations from year to year and some 
TABLE 3.4 Annual Per Capita Consumption of Major Food Groups

\begin{tabular}{|c|c|c|c|c|c|c|}
\hline \multirow[b]{2}{*}{ Year } & \multicolumn{2}{|c|}{ Crops $^{a}$} & \multicolumn{2}{|c|}{ Meat ${ }^{b}$} & \multicolumn{2}{|c|}{ Dairyc } \\
\hline & $\mathrm{lb} / \mathrm{yr}$ & $\mathrm{kg} / \mathrm{yr}$ & $\mathrm{lb} / \mathrm{yr}$ & $\mathrm{kg} / \mathrm{yr}$ & $\mathrm{lb} / \mathrm{yr}$ & $\mathrm{kg} / \mathrm{yr}$ \\
\hline $1947-1949$ & 902.2 & 409.23 & 234.8 & 106.50 & 482.6 & 218.90 \\
\hline $1957-1959$ & 807.1 & 366.09 & 245.9 & 111.54 & 470.1 & 213.23 \\
\hline $1967-1969$ & 776.0 & 351.99 & 266.5 & 120.88 & 439.6 & 199.40 \\
\hline 1970 & 779.0 & 353.35 & 273.6 & 124.10 & 432.3 & 196.09 \\
\hline 1971 & 779.3 & 353.49 & 278.8 & 126.46 & 434.0 & 196.86 \\
\hline 1972 & 779.2 & 353.44 & 276.5 & 125.42 & 435.7 & 197.63 \\
\hline 1973 & 788.1 & 357.48 & 260.5 & 118.16 & 437.6 & 198.49 \\
\hline 1974 & 772.5 & 350.40 & 270.0 & 122.47 & 421.9 & 191.37 \\
\hline 1975 & 795.2 & 360.70 & 260.0 & 117.93 & 424.0 & 192.32 \\
\hline 1976 & 809.2 & 367.05 & 272.4 & 123.56 & 437.6 & 198.49 \\
\hline 1977 & 806.5 & 365.82 & 271.8 & 123.29 & 432.6 & 196.22 \\
\hline 1978 & 802.8 & 364.14 & 269.9 & 122.43 & 431.9 & 195.91 \\
\hline 1979 & 820.2 & 372.04 & 273.0 & 123.83 & 431.4 & 195.68 \\
\hline 1980 & 824.3 & 373.90 & 275.8 & 125.10 & 425.4 & 192.96 \\
\hline 1981 & 818.1 & 371.08 & 274.0 & 124.28 & 419.7 & 190.37 \\
\hline 1982 & 830.1 & 376.53 & 268.4 & 121.74 & 428.1 & 194.18 \\
\hline 1983 & 843.1 & 382.42 & 275.3 & $124.8 T$ & 438.2 & 198.76 \\
\hline 1984 & 841.1 & 381.52 & 277.3 & 125.78 & 447.5 & 202.98 \\
\hline
\end{tabular}

${ }^{a}$ Crops include vegetables; dried beans, peas, nuts, and soya products; flour and cereal products; sugar and other sweeteners (includes refined sugar, corn sweeteners, molasses, honey, maple, and other syrups); coffee, tea, and cucoa (including regular and instant coffee on a roasted basis, tea, spices, and chocolate 1 iquor equivalent of cocoa and chocolate products); spices and herbs; butter/ margarine; shortening; edible oils; and fruits. Crops are on a product-weight basis except for concentrated juices, which are on a single-strength equivalent basis. Also includes home garden produce of Eruits, vegetables, potatoes, dried beans, and iried peas.

beat includes meat counted on a fresh-retail-cut equivalent basis (including fat cuts of pork, game, and edible offal); poultry (excluding game birds) counted on a ready-to-cook basis; fish (including game fish) counted on; eggs counced on a shell-egg basis; and lard and beef tallow.

${ }^{C}$ Dairy includes fluid milk (based on calcium equivalent of whole milk and creams); canned, evaporated, and dry milk; whey; ice cream and other frozen desserts; and yogurt.

Source: USDA (1986). 
apparent trends in consumption. It is also recommended that any modeling cependent on food chain uptake rely on these numbers in estimating the uptake by individuals and populations. For 1984, per capita consumption was crops, $841.1 \mathrm{lb}$; meat, $277.3 \mathrm{lb}$; and dairy, $447.5 \mathrm{lb}$. These numbers are inherently conservative, however, because they are based on production divided by population, not by actual consumption.

Because actual consumption numbers are unavailable, a technique for deriving more-accurate consumption data from the data in Table 3.4 has been suggested by Hanson (1989). He recommends using the ratio of calories produced to calories consumed as a basis for moving from production to consumption. He estimates 3,600 calories per person per day are produced, with 2,000 calories per person per day actually consumed. Thus, to use this approach, any number in Table 3.4 should be multiplied by 0.56 ; the resulting consumption data for 1984 are crops, $471.01 \mathrm{lb}$; meat, $155.29 \mathrm{lb}$; and dairy, $250.6 \mathrm{lb}$. (Alcohol consumption is not believed to be accurately reported, so the caloric ratios estimated by Hanson may be understating "ingestion.") The recommended multiplier constitutes only a basis for determining consumption for all agricultural commodities. The actual multiplier within one of these commodities may be different than the multiplier for another commodity; thus, the multiplier used in this model represents a summary measure of actual consumption.

\subsection{USE OF THE AGRICULTURAL DATA IN TRANSPORTATION ACCIDENT MODELING}

A number of potential problems may arise when the sum mary statistics presented in Tables 3.2 and 3.3 are used in route-specific analyses of the risks associated with SNF and HLW transport. The first is that agricultural production is not of ten evenly distributed across a given state. Thus, using average statistics per area either overestimates or underestimates the production in any one lucation. In addition, the agricultural export economy means that production is not necessarily consumed in-state. One should be careful in making any assumptions that would have local residents consuming all state-produced agricultural products. However, the problem remains for modelers regarding how to distribute the impacts to the food chain over the population. This problem is complicated by the fact that agricultural products grown in one state may be processed in another state and then distributed regionally, nationally, or internationally. The originating state may receive only a small portion of the final product. The exception to this is on-farm consumption. The more site-specific the modeling, the more detailed the agricultural statistics must be. The data presented here are suitable for use at the national level. It is suggested that county-level agricultural production be calculated using the approach presented here for modeling at the regional or state level. In addition, digitized maps indicating agricultural production areas should be considered along with growing-season information in order to more accurately identify agricultural production along transportation routes. Data are currently available at the county level. New data were collected in 1987 but were not published at the time this report was written. The data in Tables 3.2 and 3.3 should be recalculated based on 1987 data, when available. 
The USDA estimates of per capita consumption of crops, meat, and dairy products (Table 3.4) can be used as one means to calculate impacts on an individual or a population from food chain uptake. In situations where the amount of contaminated farm tand is known or projected, per capita consumption can be used as a way of determining the minimum number of people likely to be affected by the contaminated food. The minimum number of people affected (assuming that they received their total food input from the contaminated area) can be calculated by:

$$
\text { number of people affected }=\frac{\text { number of acres affected } \times \text { production/acre }}{\text { per capita consumption/year }}
$$

This approach would be independent of state population.

Given these recommendations, it is anticipated the agricultural statistics data presented in t'is report can have wide applicability in models dealing with production, movement/transportation, and disposal of a variety of hazardous substances at the national level. 


\section{REFERENCES}

ALK Associates, 1986, Railroad Traffic in the United States, 1985 Ed., Princeton, N.J. (Aug.).

ALK Associates, 1988, Railroad Traffic in the United States, 1986 Ed., Princeton, N.J. (Feb.).

American Meat Institute, 1986, Meatfacts, 16 th Annual Edition (July).

Association of American Kailroads, 1986, Railroad Facts, Office of Information and Public Affairs, Washington, D.C.

Association of American Railroads, 1987, Railroad Facts, Office of Information and Public Affairs, Washington, D.C.

Battelle Memorial Institute, 1989, unpublished information, Office of Transportation Systems and Plenning.

Federal Highway Administration, 1987, Highway Statistics 1986, Publ. No. HHP-41, Washington, D.C.

Hanson, G., 1989, Professor Emeritus, Department of Food Science and Nutrition, Utah State University, Logan, personal communication to R.G. Williams (July 26).

Jahnke, D., 1987, U.S. Bureau of the Census, Agriculture Division, Suitland, Md., personal communication to R.G. Williams (Feb.-May).

Madsen, M.M., J.M. Taylor, and R.M. Ostemeyer, 1986, RADTRAN III, SAND84-0036, Sandia National Laboratories, Albuquerque, N.M.

Peterson, B.E., and F. Southworth, 1984, Disaggregation of Highway Traffic by Vehicle Type, prepared by Oak Ridge National Laboratory, Oak Ridge, Tenn., for Federal Highway Administration, Office of Program and Policy Planning.

Sitzman, R., 1987, U.S. Department of Agriculture, National Agricultural Statistical Service, Washington, D.C., personal communication to R.G. Williams (March 26).

Tobin, R.L., N.K. Meshkov, and R.H. .7nes, 1985, Preliminary Assessment of the Costs and Risks of Transporting Spent Fuel by Barge, Argonne National Laboratory Report ANL/ER-TM-85-2 (Dec.).

U.S. Army Corps of Engineers, 1987, Waterborne Commerce of the United States Calendrar Year 1985, Report No. WRSC-WCUS-85-(1-5), New Orleans, La.

J.S. Bureau of the Census, 1982, Truck Inventory and Use Survey, TC82-T-52, Washington, D.C. (Public-Use Tapes). 


\section{$33 / 34$}

U.S. Bureau of the Census, 1984, 1982 Census of Agriculture, Volume 1, Geographic Series, Part 51, United $\leq$ ates Summary and State Data, AC82-A-51 (Oct.).

U.S. Coast Guard, 1984, Coding Instructions for the Automated File of Commercial Vessel Casualties, Washington, D.C. (Feb.).

U.S. Department of Agriculture, 1983, Poultry Slaughter, Annual Revision Tables, Statistical Reporting Service, Crop Reporting Board (April).

U.S. Department of Agriculture, 1985a, Chicken and Eggs, Final Estimates for 1980-83, Statistical Bulletin 721, Statistical Reporting Service, Crop Reporting Board (Jan).

U.S. Department of Agriculture, 1985b, Milk Final Estimates 1979-82, Statistical Bulletin 722, Statistica! Reporting Service, Crop Reporting Board (Feb).

U.S. Department of Agriculture, 1985c, Poultry Production, Disposition, and Income Final Estimates for 1980-83, Statistical Bulletin 725, Statistical Reporting Service, Crop Reporting Board (April).

U.S. Department of Agriculture, 1986, Nutrient Content of the Food Supply and a Table of Food Consumption per Capita for 1947-49, 1957-59, 1967-69 and 1970-84, HNIS (Adm.299-19), Human Nutrition Information Service (June).

U.S. Department of Energy, 1986a, Environmental Assessment, Deaf Smith Site, Texas, DOE/RW-0069 (Vols. 1-3), Office of Civilian Radioactive Waste Management (May).

U.S. Department of Energy, 1986b, Environmental Assessment, Reference Repository Location, Hanford Site, Washington, DOE/RW-0070 (Vols. 1-3), Office of Civilian Radioactive Waste Management (May).

U.S. Department of Energy, 1986c, Environmental Assessment, Yucca Mountain Site, Nevada Research and Development Area, Nevada, DOE/RW-0071 (Vols. 1-3), Office of Civilian Radioactive Waste Management (May).

U.S. Department of Transportation, Accident/Incident Bulletin: No. 155, Federal Railroad Administration Office of Safety (July 1987).

Willekens, F., 1985, Fiybrid Log-Linear Models, in P. Nijkamp, H. Leitner, and N. Wrigley (Eds.), Measuring the Unmeasurable, Martinus Nijhoff Publishers, Boston, pp. 141-176. 
APPENDIX A:

STATE AGRICULTURAL PRODUCTION STATISTICS, 1982 
'PABLl A.i State Agricultura' Production, 1982: Crops (spreadsheet columns are given for ease of reference to Appendix $B$ )

\begin{tabular}{|c|c|c|c|c|c|c|}
\hline \multirow[b]{2}{*}{$\begin{array}{l}\text { State } \\
(\text { Col. A) }\end{array}$} & \multicolumn{2}{|c|}{ Corn } & \multicolumn{2}{|c|}{ Wheat } & \multicolumn{2}{|c|}{ Soybeans } \\
\hline & $\begin{array}{c}b^{a} \\
(\operatorname{col} \cdot Q)\end{array}$ & $\begin{array}{c}l b \\
(\operatorname{Col} \cdot R)\end{array}$ & $\begin{array}{c}\text { bu } \\
(\mathrm{Col} . \mathrm{s})\end{array}$ & $\begin{array}{c}1 \mathrm{~b} \\
(\operatorname{col} \cdot \mathrm{T})\end{array}$ & $\begin{array}{c}\text { bu } \\
\text { (Col. U) }\end{array}$ & $\begin{array}{c}1 b \\
(\operatorname{col} \cdot v)\end{array}$ \\
\hline Al abama & $2.01 \times 10^{7}$ & $1.13 \times 10^{9}$ & $1.41 \times 10^{7}$ & $8.44 \times 10^{\circ}$ & $3.64 \times 10^{7}$ & $2.18 \times 10^{9}$ \\
\hline Al aska & $-b$ & - & $2.80 \times 10^{3}$ & $1.68 \times 10^{5}$ & - & - \\
\hline Arizona & $1.50 \times 10^{6}$ & $8.39 \times 10^{7}$ & $1.19 \times 10^{7}$ & $7.13 \times 10^{8}$ & - & - \\
\hline Arkansas & $2.39 \cdot 10^{6}$ & $1.34 \times 10^{8}$ & $6.13 \times 10^{7}$ & $3.68 \times 10^{9}$ & $9.86 \times 10^{7}$ & $5.92 \times 10^{9}$ \\
\hline Cal ifornia & $3.77 \times 10^{7}$ & $2.11 \times 10^{9}$ & $6.31 \times 10^{7}$ & $3.79 \times 10^{9}$ & $1.71 \times 10^{4}$ & $1.02 \times 10^{6}$ \\
\hline Colorado & $9.49 \times 10^{7}$ & $5.31 \times 10^{9}$ & $7.83 \times 10^{7}$ & $4.70 \times 10^{9}$ & $2.88 \times 10^{4}$ & $1.73 \times 10^{6}$ \\
\hline Connecticut & $6.28 \times 10^{5}$ & $3.52 \times 10^{7}$ & $8.14 \times 10^{3}$ & $4.88 \times 10^{5}$ & $1.28 \times 10^{3}$ & $7.69 \times 10^{3}$ \\
\hline Delaware & $1.49 \times 10^{7}$ & $8.33 \times 10^{8}$ & $2.23 \times 10^{6}$ & $1.34 \times 10^{8}$ & $6.37 \times 10^{6}$ & $3.82 \times 10^{8}$ \\
\hline Florida & $1.26 \times 10^{7}$ & $7.03 \times 10^{8}$ & $2.77 \times 10^{6}$ & $1.66 \times 10^{8}$ & $8.53 \times 10^{6}$ & $5.12 \times 10^{8}$ \\
\hline Georgia & $6.00 \times 10^{7}$ & $3.36 \times 10^{9}$ & $3.19 \times 10^{?}$ & $1.91 \times 10^{9}$ & $5.20 \times 10^{7}$ & $3.12 \times 10^{9}$ \\
\hline Hawa i i & - & - & - & - & - & - \\
\hline Idaho & $8.08 \times 10^{6}$ & $4.53 \times 10^{8}$ & $8.80 \times 10^{7}$ & $5.28 \times 10^{9}$ & - & - \\
\hline Ill inois & $1.43 \times 10^{9}$ & $7.98 \times 10^{10}$ & $6.20 \times 10^{7}$ & $3.72 \times 10^{9}$ & $3.36 \times 10^{8}$ & $2.02 \times 10^{10}$ \\
\hline Indiana & $7.12 \times 10^{8}$ & $3.99 \times 10^{10}$ & $3.70 \times 10^{7}$ & $2.22 \times 10^{9}$ & $1.61 \times 10^{8}$ & $9.69 \times 10^{9}$ \\
\hline Iowa & $4.67 \times 10^{8}$ & $8.21 \times 10^{10}$ & $2.85 \times 10^{6}$ & $1.71 \times 10^{8}$ & $2.91 \times 10^{8}$ & $1.75 \times 10^{10}$ \\
\hline Kansas & $1.31 \times 10^{8}$ & $7.32 \times 10^{9}$ & $3.73 \times 10^{8}$ & $2.24 \times 10^{10}$ & $4.30 \times 10^{7}$ & $2.58 \times 10^{9}$ \\
\hline Kentucky & $1.32 \times 10^{8}$ & $7.37 \times 10^{9}$ & $1.97 \times 10^{7}$ & $1.18 \times 10^{9}$ & $4.43 \times 10^{7}$ & $2.66 \times 10^{9}$ \\
\hline Louisiana & $2.77 \times 10^{6}$ & $1.55 \times 10^{8}$ & $1.35 \times 10^{7}$ & $8.11 \times 10^{8}$ & $6.77 \times 10^{7}$ & $4.06 \times 10^{9}$ \\
\hline Maine & $6.48 \times 10^{5}$ & $3.63 \times 10^{7}$ & $3.54 \times 10^{4}$ & $2.12 \times 10^{6}$ & $2.99 \times 10^{3}$ & $1.79 \times 10^{5}$ \\
\hline Maryland & $6.28 \times 10^{7}$ & $3.52 \times 10^{9}$ & $5.66 \times 10^{6}$ & $3.38 \times 10^{8}$ & $1.13 \times 10^{7}$ & $6.77 \times 1.0^{8}$ \\
\hline Massachusetrs & $5.92 \times 10^{5}$ & $3.31 \times 10^{7}$ & $1.28 \times 10^{3}$ & $7.66 \times 10^{4}$ & - & - \\
\hline Michigan & $2.56 \times 10^{8}$ & $1.44 \times 10^{10}$ & $2.08 \times 10^{7}$ & $1.25 \times 10^{9}$ & $3.43 \times 10^{7}$ & $2.06 \times 10^{9}$ \\
\hline Minnesota & $6.10 \times 10^{8}$ & $3.42 \times 10^{10}$ & $1.12 \times 10^{8}$ & $6.69 \times 10^{9}$ & $1.51 \times 10^{8}$ & $9.07 \times 10^{9}$ \\
\hline Mississippi & $4.76 \times 10^{6}$ & $2.66 \times 10^{8}$ & $2.92 \times 10^{7}$ & $1.75 \times 10^{9}$ & $8.19 \times 10^{7}$ & $4.92 \times 10^{9}$ \\
\hline Missouri & $1.79 \times 10^{8}$ & $1.00 \times 10^{10}$ & $6.83 \times 10^{7}$ & $4.10 \times 10^{9}$ & $1.54 \times 10^{8}$ & $9.22 \times 10^{9}$ \\
\hline Montana & $1.93 \times 10^{6}$ & $1.08 \times 10^{8}$ & $1.59 \times 10^{8}$ & $9.55 \times 10^{9}$ & $1.49 \times 10^{4}$ & $8.95 \times 10^{5}$ \\
\hline
\end{tabular}




\begin{tabular}{|c|c|c|c|c|c|c|}
\hline \multirow[b]{2}{*}{$\begin{array}{l}\text { State } \\
\text { (Col. A) }\end{array}$} & \multicolumn{2}{|c|}{ Corn } & \multicolumn{2}{|c|}{ Wheat } & \multicolumn{2}{|c|}{ Soybeans } \\
\hline & $\begin{array}{c}\text { bu } \\
(\mathrm{Col} \cdot \mathrm{Q})\end{array}$ & $\begin{array}{c}1 b \\
(\operatorname{Col} \cdot \mathrm{R})\end{array}$ & $\begin{array}{c}\text { bu } \\
(\mathrm{Col} \cdot \mathrm{s})\end{array}$ & $\begin{array}{c}1 \mathrm{~b} \\
(\mathrm{Col} \cdot \mathrm{T})\end{array}$ & $\begin{array}{c}\text { bu } \\
\text { (Col. U) }\end{array}$ & $\begin{array}{c}1 b \\
(\mathrm{Col} \cdot \mathrm{V})\end{array}$ \\
\hline Nebraska & $6.76 \times 10^{8}$ & $3.79 \times 10^{10}$ & $8.80 \times 10^{7}$ & $5.28 \times 10^{9}$ & $7.02 \times 10^{7}$ & $4.21 \times 10^{9}$ \\
\hline Nevada & - & - & $1.39 \times 10^{6}$ & $8.32 \times 10^{7}$ & - & - \\
\hline New Hampshire & $1.46 \times 10^{5}$ & $8.16 \times 10^{6}$ & - & - & - & - \\
\hline New Jersey & $1.02 \times 10^{7}$ & $5.70 \times 10^{8}$ & $1.46 \times 10^{6}$ & $8.77 \times 10^{7}$ & $4.11 \times 10^{6}$ & $2.47 \times 10^{8}$ \\
\hline New Mexico & $6.90 \times 10^{6}$ & $3.86 \times 10^{8}$ & $1.00 \times 10^{7}$ & $6.02 \times 10^{8}$ & $4.28 \times 10^{4}$ & $2.57 \times 10^{6}$ \\
\hline New York & $7.03 \times 10^{7}$ & $3.94 \times 10^{9}$ & $4.95 \times 10^{6}$ & $2.97 \times 10^{8}$ & $5.84 \times 10^{5}$ & $3.50 \times 10^{7}$ \\
\hline North Carolina & $1.34 \times 10^{8}$ & $7.48 \times 10^{9}$ & $2.07 \times 10^{7}$ & $1.24 \times 10^{9}$ & $4.23 \times 10^{7}$ & $2.54 \times 10^{9}$ \\
\hline North Dakota & $3.41 \times 10^{7}$ & $1.91 \times 10^{9}$ & $2.96 \times 10^{8}$ & $1.78 \times 10^{10}$ & $8.80 \times 10^{6}$ & $5.28 \times 10^{8}$ \\
\hline Ohio & $4.18 \times 10^{8}$ & $2.34 \times 10^{10}$ & $4.74 \times 10^{7}$ & $2.85 \times 10^{9}$ & $1.24 \times 10^{8}$ & $7.46 \times 10^{9}$ \\
\hline Oklahoma & $3.65 \times 10^{6}$ & $2.05 \times 10^{8}$ & $1.72 \times 10^{8}$ & $1.03 \times 10^{10}$ & $4.63 \times 10^{6}$ & $2.78 \times 10^{8}$ \\
\hline Oregon & $7.19 \times 10^{6}$ & $4.03 \times 10^{8}$ & $5.89 \times 10^{7}$ & $3.54 \times 10^{9}$ & - & - \\
\hline Pennsyluania & $1.20 \times 10^{8}$ & $6.71 \times 10^{9}$ & $7.79 \times 10^{6}$ & $4.67 \times 10^{8}$ & $3.83 \times 10^{6}$ & $2.30 \times 10^{8}$ \\
\hline Rhode Island & $1.09 \times 10^{4}$ & $6.11 \times 10^{5}$ & - & - & - & - \\
\hline South Carolina & $3.11 \times 10^{7}$ & $1.74 \times 10^{9}$ & $1.48 \times 10^{7}$ & $8.89 \times 10^{8}$ & $2.90 \times 10^{7}$ & $1.74 \times 10^{9}$ \\
\hline South Dakota & $1.74 \cdot 10^{8}$ & $9.75 \times 10^{9}$ & $8.59 \times 10^{7}$ & $5.15 \times 10^{9}$ & $2.23 \times 10^{7}$ & $1.34 \times 10^{9}$ \\
\hline Tennessee & $4.85 \times 10^{7}$ & $2.72 \times 10^{9}$ & $2.50 \times 10^{7}$ & $1.50 \times 10^{9}$ & $5.54 \times 10^{7}$ & $3.32 \times 10^{y}$ \\
\hline Texas & $1.10 \times 10^{8}$ & $6.17 \times 10^{9}$ & $1.23 \times 10^{8}$ & $7.38 \times 10^{9}$ & $1.78 \times 10^{7}$ & $1.07 \times 10^{9}$ \\
\hline Utah & $1.62 \times 10^{6}$ & $9.06 \times 10^{7}$ & $6.66 \times 10^{6}$ & $4.00 \times 10^{8}$ & - & - \\
\hline Veimont & $1.17 \times 10^{6}$ & $6.57 \times 10^{7}$ & $1.04 \times 10^{4}$ & $6.22 \times 10^{5}$ & - & - \\
\hline Virginia & $5.83 \times 10^{7}$ & $3.26 \times 10^{9}$ & $1.09 \times 10^{7}$ & $6.52 \times 10^{8}$ & $1.77 \times 10^{7}$ & $1.06 \times 10^{9}$ \\
\hline Washir zton & $2.08 \times 10^{7}$ & $1.17 \times 10^{9}$ & $1.28 \times 10^{8}$ & $7.68 \times 10^{9}$ & - & - \\
\hline West Virginia & $7.42 \times 10^{6}$ & $4.15 \times 10^{8}$ & $2.44 \times 10^{5}$ & $1.46 \times 10^{7}$ & $1.63 \times 10^{5}$ & $9.76 \times 10^{6}$ \\
\hline Wisconsin & $3.32 \times 10^{8}$ & $1.86 \times 10^{10}$ & $5.51 \times 10^{6}$ & $3.30 \times 10^{8}$ & $1.06 \times 10^{7}$ & $6.37 \times 10^{8}$ \\
\hline Wyoming & $4.68 \times 10^{6}$ & $2.62 \times 10^{8}$ & $9.17 \times 10^{6}$ & $5.50 \times 10^{8}$ & - & - \\
\hline U.S. Total & $7.51 \times 10^{9}$ & $4.20 \times 10^{11}$ & $2.37 \times 10^{9}$ & $1.42 \times 10^{11}$ & $1.99 \times 10^{9}$ & $1.19 \times 10^{11}$ \\
\hline
\end{tabular}




\begin{tabular}{|c|c|c|c|c|c|}
\hline \multirow[b]{2}{*}{$\begin{array}{l}\text { State } \\
\text { (Col.A) }\end{array}$} & \multicolumn{2}{|c|}{ Potatoes } & Honey & \multicolumn{2}{|c|}{ Sorghum } \\
\hline & $\begin{array}{c}\operatorname{cwt}^{c} \\
(\operatorname{Col} \cdot W)\end{array}$ & $\begin{array}{c}1 b \\
(\operatorname{Col} \cdot x)\end{array}$ & $\begin{array}{c}\mathrm{lb} \\
(\mathrm{Col} \cdot \mathrm{Y})\end{array}$ & $\begin{array}{c}\text { bu } \\
(\mathrm{Col} \cdot \mathrm{z})\end{array}$ & $\begin{array}{c}1 \mathrm{~b} \\
(\mathrm{Col} \cdot \mathrm{AA})\end{array}$ \\
\hline Al abama & $2.03 \times 10^{6}$ & $2.03 \times 10^{8}$ & $1.35 \times 10^{6}$ & $2.12 \times 10^{6}$ & $1.19 \times 10^{8}$ \\
\hline Alaska & $8.23 \times 10^{4}$ & $8.23 \times 10^{6}$ & $3.15 \times 10^{3}$ & - & - \\
\hline Arizona & $1.21 \times 10^{6}$ & $1.21 \times 10^{8}$ & $3.18 \times 10^{6}$ & $8.33 \times 10^{5}$ & $4.67 \times 10^{7}$ \\
\hline Arkansas & $1.97 \times 10^{4}$ & $1.97 \times 10^{6}$ & $1.55 \times 10^{6}$ & $1.39 \times 10^{7}$ & $7.77 \times 10^{8}$ \\
\hline California & $1.79 \times 10^{7}$ & $1.79 \times 10^{9}$ & $2.51 \times 10^{7}$ & $3.97 \times 10^{6}$ & $2.22 \times 10^{8}$ \\
\hline Colorado & $1.48 \times 10^{7}$ & $1.49 \times 10^{9}$ & $2.02 \times 10^{6}$ & $9.82 \times 10^{6}$ & $5.50 \times 10^{8}$ \\
\hline Connecticut & $3.67 \times 10^{5}$ & $3.67 \times 10^{7}$ & $6.60 \times 10^{4}$ & - & - \\
\hline Delaware & $1.33 \times 10^{6}$ & $1.33 \times 10^{8}$ & $1.33 \times 10^{4}$ & $4.41 \times 10^{2}$ & $2.47: 10^{5}$ \\
\hline Florida & $6.53 \times 10^{6}$ & $6.53 \times 10^{8}$ & $1.16 \times 10^{7}$ & $7.69 \times 10^{5}$ & $4.31 \times 10^{7}$ \\
\hline Ceorgia & $1.00 \times 10^{5}$ & $1.00 \times 10^{6}$ & $3.54 \times 10^{6}$ & $3.98 \times 10^{6}$ & $2.23 \times 10^{8}$ \\
\hline Hawai i & $1.44 \times 10^{3}$ & $1.44 \times 10^{5}$ & $7.77 \times 10^{5}$ & - & - \\
\hline Idaho & $8.95 \times 10^{7}$ & $8.95 \times 10^{9}$ & $3.87 \times 10^{6}$ & - & - \\
\hline Il inois & $5.80 \times 10^{5}$ & $5.80 \times 10^{7}$ & $6.37 \times 10^{5}$ & $8.25 \times 10^{6}$ & $4.62 \times 10^{8}$ \\
\hline Indiana & $9.08 \times 10^{5}$ & $9.08 \times 10^{7}$ & $3.01 \times 10^{5}$ & $3.43 \times 10^{5}$ & $1.92 \times 10^{7}$ \\
\hline lowa & $2.88 \times 10^{5}$ & $2.88 \times 10^{7}$ & $2.73 \times 10^{6}$ & $7.53 \times 10^{5}$ & $4.22 \times 10^{7}$ \\
\hline Kansas & $1.45 \times 10^{5}$ & $1.45 \times 10^{7}$ & $1.13 \times 10^{6}$ & $1.92 \times 10^{8}$ & $1.08 \times 10^{10}$ \\
\hline Kentucky & $3.48 \times 10^{5}$ & $3.48 \times 10^{7}$ & $1.20 \times 10^{5}$ & $2.03 \times 10^{6}$ & $1.13 \times 10^{8}$ \\
\hline Louisiana & $5.35 \times 10^{4}$ & $5.35 \times 10^{6}$ & $2.34 \times 10^{6}$ & $6.23 \times 10^{0}$ & $3.49 \times 10^{8}$ \\
\hline Maine & $2.46 \times: 0^{7}$ & $2.46 \times 10^{9}$ & $3.23 \times 10^{5}$ & - & - \\
\hline Maryl and & $3.96 \times 10^{5}$ & $3.96 \times 10^{7}$ & $9.08 \times 10^{4}$ & $7.48 \times 10^{4}$ & $4.19 \times 10^{6}$ \\
\hline Massachusetts & $7.33 \times 10^{5}$ & $7.33 \times 10^{7}$ & $1.23 \times 10^{5}$ & - & - \\
\hline Michigan & $1.24 \times 10^{7}$ & $1.24 \times 10^{9}$ & $4.38 \times 10^{6}$ & $4.88 \times 10^{4}$ & $2.73 \times 10^{6}$ \\
\hline Minnesota & $1.41 \times 10^{7}$ & $1.41 \times 10^{9}$ & $1.08 \times 10^{7}$ & $9.86 \times 10^{4}$ & $5.52 \times 10^{6}$ \\
\hline Mississippi & $3.46 \times 10^{4}$ & $3.46 \times 10^{6}$ & $1.66 \times 10^{6}$ & $4.82 \times 10^{6}$ & $2.70 \times 10^{8}$ \\
\hline Missouri & $2.70 \times 10^{5}$ & $2.70 \times 10^{7}$ & $9.62 \times 10^{5}$ & $5.49 \times 10^{7}$ & $3.07 \times 10^{9}$ \\
\hline Montana & $1.89 \times 10^{6}$ & $1.89 \times 10^{8}$ & $1.01 \times 10^{7}$ & - & - \\
\hline Nebraska & $2.01 \times 10^{6}$ & $2.01 \times 10^{8}$ & $5.43 \times 10^{6}$ & $1.17 \times 10^{8}$ & $6.55 \times 10^{9}$ \\
\hline
\end{tabular}


TABLI: A.1 (Cont'd)

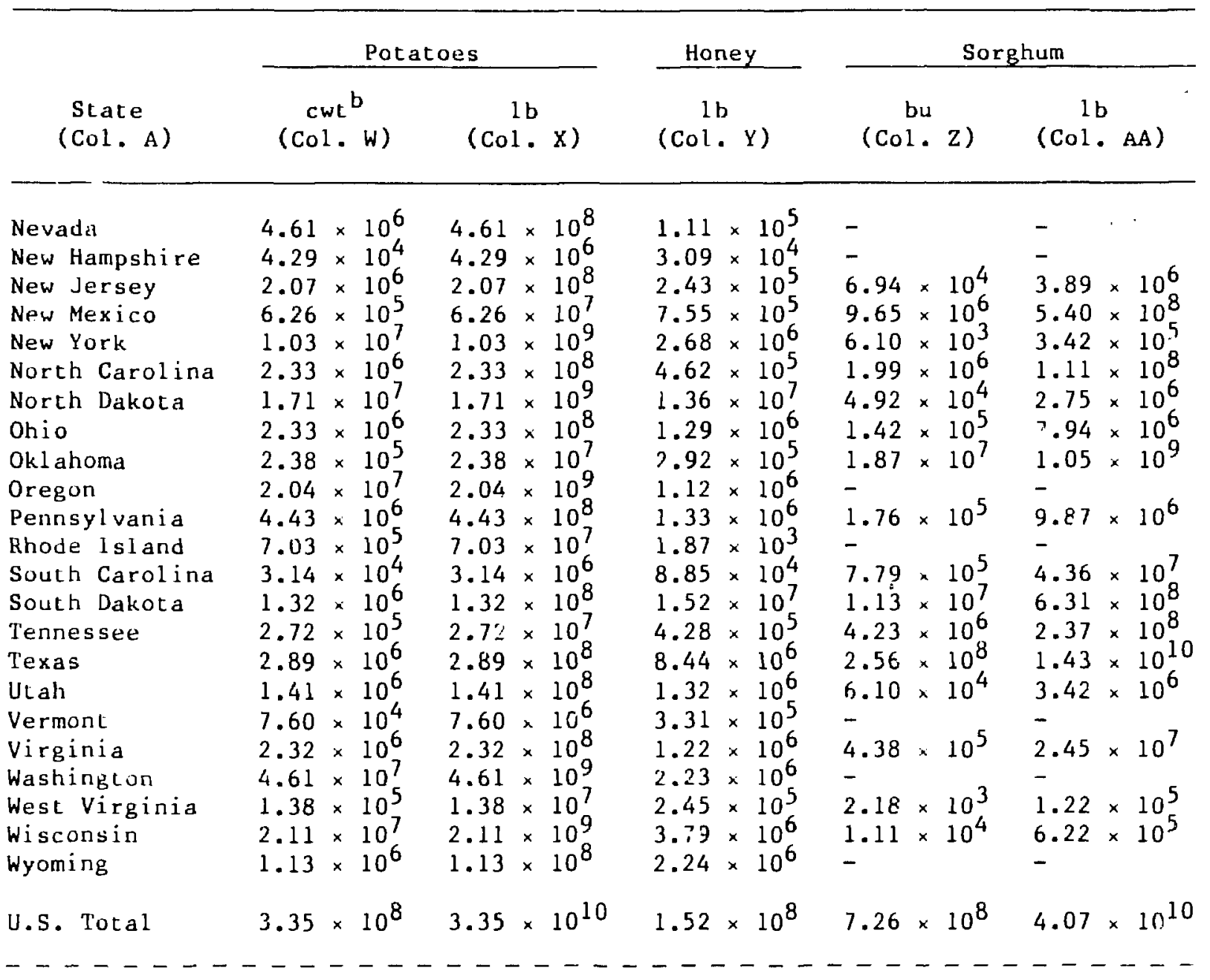




\begin{tabular}{|c|c|c|c|c|c|c|}
\hline \multirow[b]{2}{*}{$\begin{array}{l}\text { State } \\
(\text { Col. A })\end{array}$} & \multicolumn{2}{|c|}{ Barley } & \multicolumn{2}{|c|}{ Oats } & \multicolumn{2}{|c|}{ Rye } \\
\hline & $(\mathrm{Col} \cdot \mathrm{AB})$ & $\begin{array}{c}1 \mathrm{~b} \\
(\mathrm{Col} \cdot \mathrm{AC})\end{array}$ & $\begin{array}{c}\text { bu } \\
(\mathrm{Col} \cdot \mathrm{AD})\end{array}$ & $\left(\begin{array}{c}1 b \\
\mathrm{Col} \cdot \mathrm{AE})\end{array}\right.$ & $\begin{array}{c}\text { bu } \\
(\mathrm{Col} \cdot \mathrm{AF})\end{array}$ & $\begin{array}{c}1 \mathrm{~b} \\
(\mathrm{Col} \cdot \mathrm{AG})\end{array}$ \\
\hline Al abama & $4.51 \times 10^{4}$ & $2.17 \times 10^{6}$ & $1.69 \times 10^{6}$ & $5.39 \times 10^{7}$ & $1.25 \times 10^{5}$ & $7.02 \times 10^{6}$ \\
\hline Alaska & $2.28 \times 10^{5}$ & $1.09 \times 10^{7}$ & $2.96 \times 10^{4}$ & $9.46 \times 10^{5}$ & - & - \\
\hline Arizona & $3.33 \times 10^{6}$ & $1.60 \times 10^{8}$ & $4.92 \times 10^{5}$ & $1.58 \times 10^{7}$ & - & - \\
\hline Arkansas & $2.42 \times 10^{4}$ & $1.16 \times 10^{6}$ & $2.36 \times 10^{6}$ & $7.5^{\prime}: \times 10^{7}$ & $2.88 \times 10^{4}$ & $1.61 \times 10^{6}$ \\
\hline California & $3.20 \times 10^{7}$ & $1.54 \times 10^{9}$ & $3.32 \times 10^{6}$ & $1.06 \times 10^{8}$ & $2.68 \times 10^{4}$ & $1.50 \times 10^{6}$ \\
\hline Colorado & $1.39 \times 10^{7}$ & $6.68 \times 10^{8}$ & $1.85 \times 10^{6}$ & $5.92 \times 10^{7}$ & $1.98 \times 10^{4}$ & $1.11 \times 10^{6}$ \\
\hline Connecticut & - & - & $1.53 \times 10^{4}$ & $4.90 \times 10^{5}$ & $2.15 \times 10^{4}$ & $1.21 \times 10^{6}$ \\
\hline Delaware & $1.91 \times 10^{6}$ & $9.18 \times 10^{7}$ & $6.70 \times 10^{4}$ & $2.15 \times 10^{6}$ & $3.63 \times 10^{4}$ & $2.03 \times 10^{6}$ \\
\hline Florida & - & - & $1.60 \times 10^{6}$ & $5.11 \times 10^{7}$ & $1.17 \times 10^{5}$ & $6.54 \times 10^{6}$ \\
\hline Ceorgia & $8.83 \times 10^{5}$ & $4.24 \times 10^{7}$ & $4.00 \times 10^{6}$ & $1.28 \times 10^{8}$ & $5.79 \times 10^{5}$ & $3.24 \times 10^{7}$ \\
\hline Hawa i i & - & - & - & - & - & - \\
\hline Idaho & $7.32 \times 10^{7}$ & $3.51 \times 10^{9}$ & $2.79 \times 10^{6}$ & $8.94 \times 10^{7}$ & $2.20 \times 10^{3}$ & $1.23 \times 10^{5}$ \\
\hline I 11 inois & $1.73 \times 10^{5}$ & $8.31 \times 10^{6}$ & $1.03 \times 10^{7}$ & $3.29 \times 10^{8}$ & $1.28 \times 10^{5}$ & $7.19 \times 10^{6}$ \\
\hline Indi ana & $1.64 \times 10^{5}$ & $7.86 \times 10^{6}$ & $5.87 \times 10^{6}$ & $1.88 \times 10^{8}$ & $8.15 \times 10^{4}$ & $4.56 \times 10^{6}$ \\
\hline I owa & $1.62 \times 10^{5}$ & $7.77 \times 10^{6}$ & $4.58 \times 10^{7}$ & $1.46 \times 10^{9}$ & $5.81 \times 10^{4}$ & $3.25 \times 10^{6}$ \\
\hline Kansas & $1.49 \times 10^{6}$ & $7.15 \times 10^{7}$ & $7.80 \times 10^{6}$ & $2.50 \times 10^{8}$ & $6.08 \times 10^{4}$ & $3.41 \times 10^{6}$ \\
\hline Kentucky & $1.16 \times 10^{6}$ & $5.57 \times 10^{7}$ & $2.27 \times 10^{5}$ & $7.28 \times 10^{6}$ & $1.15 \times 10^{4}$ & $6.42 \times 10^{5}$ \\
\hline Louisiana & - & - & $1.03 \times 10^{6}$ & $3.28 \times 10^{7}$ & $3.74 \times 10^{3}$ & $2.09 \times 10^{5}$ \\
\hline Maine & $3.58 \times 10^{4}$ & $1.72 \times 10^{6}$ & $2.51 \times 10^{6}$ & $8.05 \times 10^{7}$ & $2.49 \times 10^{4}$ & $1.40 \times 10^{6}$ \\
\hline Maryland & $4.25 \times 10^{6}$ & $2.1 .14 \times 10^{8}$ & $8.73 \times 10^{5}$ & $2.79 \times 10^{7}$ & $9.48 \times 10^{4}$ & $5.31 \times 10^{6}$ \\
\hline Massachuset & $1.62 \times 10^{3}$ & $7.76 \times 10^{4}$ & - & - & $2.31 \times 10^{4}$ & $1.29 \times 10^{6}$ \\
\hline Michigan & $2.07 \times 10^{6}$ & $9.95 \times 10^{7}$ & $2.48 \times 10^{7}$ & $7.93 \times 10^{8}$ & $3.92 \times 10^{5}$ & $2.19 \times 10^{7}$ \\
\hline Minnesota & $4.67 \times 10^{7}$ & $2.24 \times 10^{9}$ & $7.87 \times 10^{7}$ & $2.52 \times 10^{9}$ & $2.35 \times 10^{6}$ & $1.32 \times 10^{8}$ \\
\hline Mississippi & $5.96 \times 10^{3}$ & $2.86 \times 10^{5}$ & $6.13 \times 10^{5}$ & $1.96 \times 10^{7}$ & $4.62 \times 10^{3}$ & $2.59 \times 10^{5}$ \\
\hline Missouri & $2.79 \times 10^{5}$ & $1.34 \times 10^{7}$ & $2.84 \times 10^{6}$ & $9.09 \times 10^{7}$ & $1.35 \times 10^{4}$ & $7.55 \times 10^{5}$ \\
\hline Montana & $7.21 \times 10^{7}$ & $3.46 \times 10^{9}$ & $8.07 \times 10^{6}$ & $2.58 \times 10^{8}$ & $1.58 \times 10^{5}$ & $8.83 \times 10^{6}$ \\
\hline Nebraska & $8.98 \times 10^{5}$ & $4.31 \times 10^{7}$ & $2.22 \times 10^{7}$ & $7.09 \times 10^{8}$ & $7.43 \times 10^{5}$ & $4.16 \times 10^{7}$ \\
\hline
\end{tabular}




\section{'TABLE A.1 (Cour'd)}

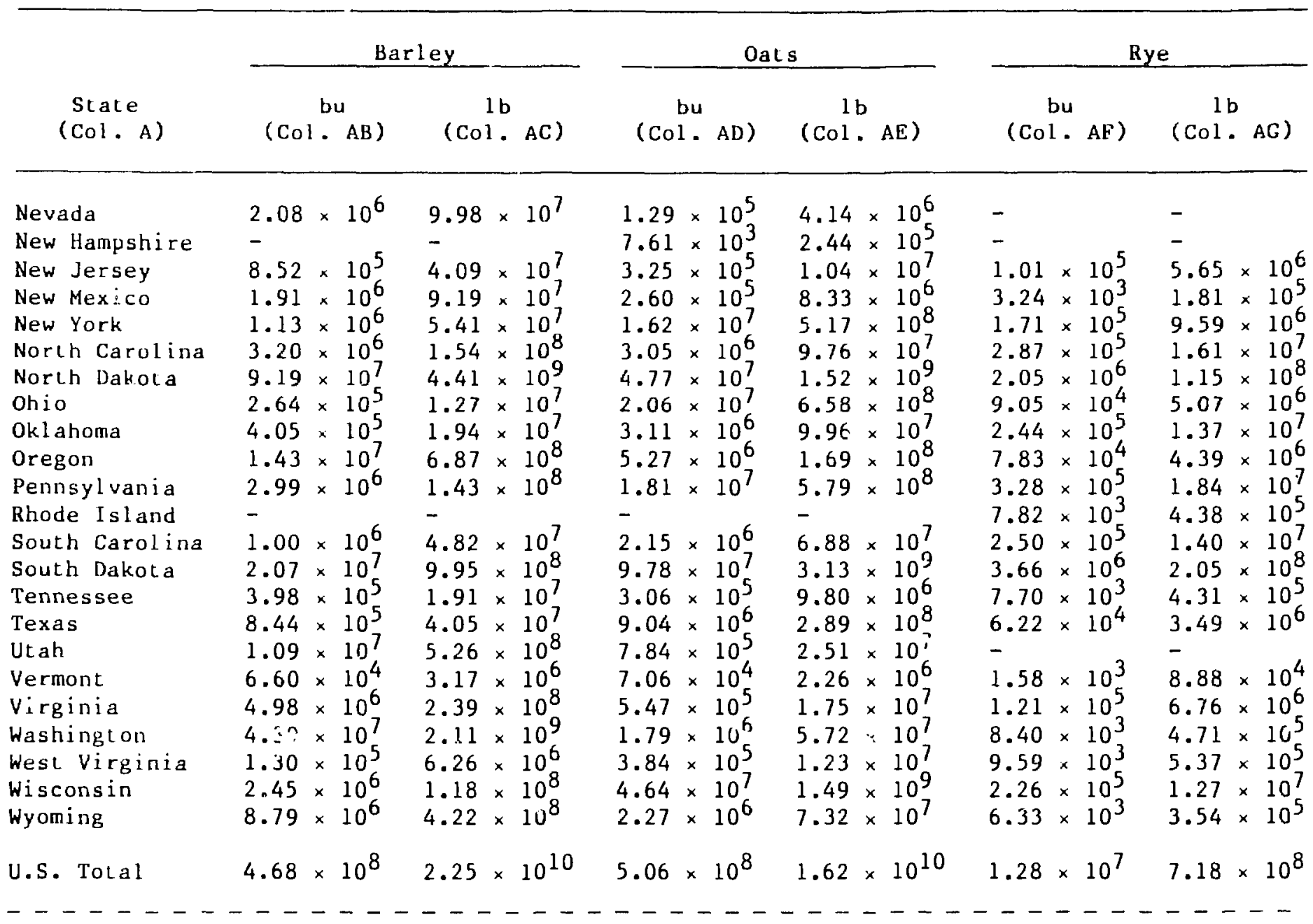


TASiLE A.1 (Cont'd)

\begin{tabular}{|c|c|c|c|c|c|c|}
\hline \multirow[b]{2}{*}{$\begin{array}{l}\text { State } \\
(\text { Col.A })\end{array}$} & \multirow{2}{*}{$\begin{array}{c}\text { Sunflowers } \\
\text { lb } \\
(\mathrm{Col} \cdot \mathrm{AH})\end{array}$} & \multicolumn{2}{|c|}{ Dry Beans } & Dry Peas & \multicolumn{2}{|c|}{ Sweet Potalues } \\
\hline & & $\begin{array}{c}c w t^{b} \\
(\mathrm{Col} \cdot A I)\end{array}$ & $\begin{array}{c}1 \mathrm{~b} \\
(\mathrm{Col} \cdot \mathrm{AJ})\end{array}$ & $(\operatorname{Col} \cdot \mathrm{AK})$ & $\begin{array}{c}\text { bu } \\
(\operatorname{Col} \cdot A L)\end{array}$ & $\begin{array}{c}l b \\
(\mathrm{Col} \cdot \mathrm{AM})\end{array}$ \\
\hline Al abama & - & - & - & $1.94 \times 10^{4}$ & $8.55 \times 10^{5}$ & $4.70 \times 10^{7}$ \\
\hline Alaska & - & - & - & - & - & - \\
\hline Arizona & - & $2.30 \times 10^{4}$ & $2.30 \times 10^{6}$ & - & - & - \\
\hline Arkansas & $2.60 \times 10^{5}$ & - & - & $8.11 \times 10^{4}$ & $7.07 \times 10^{4}$ & $3.89 \times 10^{6}$ \\
\hline Cal ifornia & $1.33 \times 10^{7}$ & $2.22 \times 10^{6}$ & $2.22 \times 10^{8}$ & $1.46 \times 10^{7}$ & $2.00 \times 10^{6}$ & $1.10 \times 10^{8}$ \\
\hline Colorado & $2.39 \times 10^{7}$ & $2.05 \times 10^{6}$ & $2.05 \times 10^{8}$ & $1.49 \times 10^{6}$ & - & - \\
\hline Connecticut & - & - & - & - & - & - \\
\hline Del aware & - & $1.90 \times 10^{3}$ & $1.90 \times 10^{5}$ & - & $1.46 \times 10^{3}$ & $8.06 \times 10^{4}$ \\
\hline Elorida & $2.66 \times 10^{6}$ & - & - & $3.79 \times 10^{5}$ & $2.82 \times 10^{5}$ & $1.55 \times 10^{7}$ \\
\hline Georgia & $2.34 \times 10^{6}$ & - & - & $1.52 \times 10^{5}$ & $8.35 \times 10^{5}$ & $4.59 \times 10^{7}$ \\
\hline Hawai i & - & - & - & - & $2.88 \times 10^{4}$ & $1.58 \times 10^{6}$ \\
\hline Idaho & $3.77 \times 10^{5}$ & $3.13 \times 10^{6}$ & $3.13 \times 10^{8}$ & $1.89 \times 10^{8}$ & - & - \\
\hline Illinois & $2.02 \times 10^{6}$ & $9.25 \times 16^{4}$ & $9.25 \times 10^{6}$ & - & $1.09 \times 10^{3}$ & $6.01 \times 10^{4}$ \\
\hline Indiana & $2.25 . .10^{6}$ & $3.94 \times 10^{4}$ & $3.94 \times 10^{6}$ & - & - & - \\
\hline Iowa & $6.42 \times 10^{5}$ & $6.91 \times 10^{4}$ & $6.91 \times 10^{6}$ & - & $1.98 \times 10^{3}$ & $1.09 \times 10^{5}$ \\
\hline Kansas & $4.85 \times 10^{7}$ & $2.62 \times 10^{5}$ & $2.62 \times 10^{7}$ & - & $1.84 \times 10^{4}$ & $1.01 \times 10^{6}$ \\
\hline Kentucky & - & - & - & - & $4.15 \times 10^{4}$ & $2.28 \times 10^{6}$ \\
\hline Louisiana & - & - & - & - & $1.54 \times 10^{6}$ & $8.49 \times 10^{7}$ \\
\hline Maine & - & - & - & $9.28 \times 10^{5}$ & - & - \\
\hline Maryl and & $7.72 \times 10^{4}$ & $2.4 i \times 10^{4}$ & $2.47 \times 10^{6}$ & - & $3.28 \times 10^{5}$ & $1.80 \times 10^{7}$ \\
\hline Massachusetts & - & - & - & - & - & - \\
\hline Michigan & $6.10 \times 10^{6}$ & $7.16 \times 10^{6}$ & $7.16 \times 10^{8}$ & - & - & - \\
\hline Minnesota & $5.17 \times 10^{8}$ & $9.46 \times 10^{5}$ & $9.46 \times 10^{7}$ & $1.72 \times 10^{6}$ & - & - \\
\hline Mississippi & - & - & - & - & $6.68 \times 10^{5}$ & $3.67 \times 10^{7}$ \\
\hline Missouri & $2.40 \times 10^{6}$ & - & - & - & $1.68 \times 10^{4}$ & $9.24 \times 10^{5}$ \\
\hline Montana & $9.78 \times 10^{6}$ & $1.12 \times 10^{5}$ & $1.12 \times 10^{7}$ & $1.27 \times 10^{6}$ & - & - \\
\hline Nebraska & $7.36 \times 10^{6}$ & $3.28 \times 10^{6}$ & $3.28 \times 10^{8}$ & - & - & - \\
\hline
\end{tabular}


TABI.E A.1 (Cont'd)

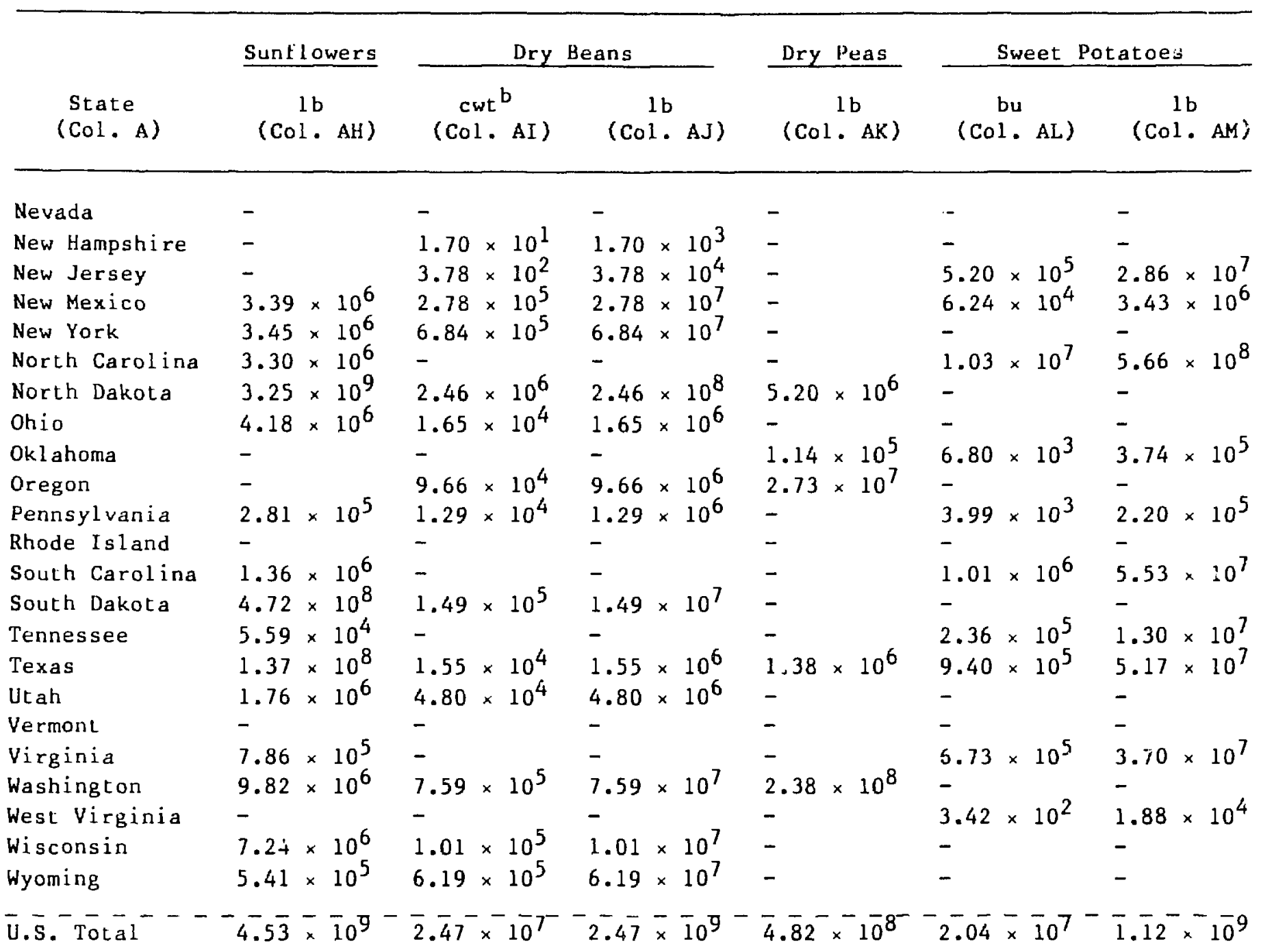


'l'ABLE A.l (Cunt'd)

\begin{tabular}{|c|c|c|c|c|c|c|}
\hline \multirow[b]{2}{*}{$\begin{array}{l}\text { State } \\
\text { (Col. A) }\end{array}$} & \multicolumn{2}{|c|}{ Sugar Beets } & \multirow{2}{*}{$\frac{\text { Heanuts }}{\begin{array}{c}1 \mathrm{~b} \\
(\mathrm{Col} . \mathrm{AP})\end{array}}$} & \multirow{2}{*}{$\frac{\text { Apples }}{\text { 1b }}$} & \multirow{2}{*}{$\begin{array}{l}\frac{\text { Cherries }}{1 \mathrm{~b}} \\
(\mathrm{Col} \cdot \mathrm{AR})\end{array}$} & \multirow{2}{*}{ 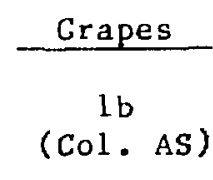 } \\
\hline & $\begin{array}{c}\text { tons } \\
(\mathrm{Col} \cdot \mathrm{AN})\end{array}$ & $\begin{array}{c}1 \mathrm{~b} \\
(\mathrm{Col} . \mathrm{AO})\end{array}$ & & & & \\
\hline $\begin{array}{l}\text { Alabama } \\
\text { Alaska }\end{array}$ & - & - & $\begin{array}{l}4.88 \times 10^{8} \\
-\end{array}$ & $\begin{array}{l}3.79 \times 10^{6} \\
-\quad-\end{array}$ & $2.58 \times 10^{2}$ & $4.31 \times 10^{5}$ \\
\hline Arizona & $2.35 \times 10^{5}$ & $4.69 \times 10^{8}$ & $1.32 \times 10^{6}$ & $4.70 \times 10^{6}$ & $3.56 \times 10^{3}$ & $2.69 \times 10^{7}$ \\
\hline Arkansas & - & - & $7.73 \times 10^{6}$ & $6.51 \times 10^{6}$ & $8.45 \times 10^{2}$ & $1.49 \times 10^{7}$ \\
\hline California & $4.27 \times 10^{6}$ & $8.55 \times 10^{9}$ & - & $3.73 \times 10^{8}$ & $2.66 \times 10^{7}$ & $9.99 \times 10^{9}$ \\
\hline Colorado & $9.15 \times 10^{5}$ & $1.83 \times 10^{9}$ & - & $4.65 \times 1 c^{7}$ & $8.38 \times 10^{5}$ & $7.41 \times 10^{4}$ \\
\hline Connecticut & - & - & - & $4.20 \times 10^{7}$ & $3.09 \times 10^{3}$ & $9.40 \times 10^{4}$ \\
\hline Delaware & - & - & - & $2.20 \times 10^{7}$ & - & $1.32 \times 10^{5}$ \\
\hline Florida & - & - & $1.38 \times 10^{8}$ & $4.37 \times 10^{4}$ & - & $1.36 \times 10^{6}$ \\
\hline Georgia & - & - & $1.38 \times 10^{9}$ & $1.28 \times 10^{7}$ & $3.26 \times 10^{2}$ & $3.52 \times 10^{6}$ \\
\hline Hawai i & - & - & .* & - & - & \\
\hline Idaho & $3.18 \times 10^{6}$ & $6.36 \times 10^{9}$ & - & $8.90 \times 10^{7}$ & $3.40 \times 10^{6}$ & $6.82 \times 10^{5}$ \\
\hline Illinois & - & - & - & $7.31 \times 10^{7}$ & $1.25 \times 10^{5}$ & $3.98 \times 10^{5}$ \\
\hline Indiana & - & - & - & $6.49 \times 10^{7}$ & $1.71 \times 10^{4}$ & $1.38 \times 10^{6}$ \\
\hline Iowa & - & - & - & $1.32 \times 10^{7}$ & $2.00 \times 10^{4}$ & $1.02 \times 10^{4}$ \\
\hline Kansas & $2.11 \times 10^{5}$ & $4.21 \times 10^{8}$ & - & $8.47 \times 10^{6}$ & $1.09 \times 10^{4}$ & $1.79 \times 10^{4}$ \\
\hline Kentucky & - & - & - & $1.02 \times 10^{7}$ & $1.07 \times 10^{3}$ & $7.97 \times 10^{4}$ \\
\hline Louisiana & - & - & $2.49 \times 10^{6}$ & $4.24 \times 10^{3}$ & - & $7.12 \times 10^{4}$ \\
\hline Maine & - & - & - & $9.03 \times 10^{7}$ & $1.66 \times 10^{3}$ & $5.72 \times 10^{3}$ \\
\hline Maryland & - & - & - & $7.92 \times 10^{7}$ & $2.87 \times 10^{5}$ & $7.21 \times 10^{5}$ \\
\hline Massachusetts & - & - & - & $9.20 \times 10^{7}$ & $2.22 \times 10^{3}$ & $1.73 \times 10^{5}$ \\
\hline Michigan & $1.81 \times 10^{6}$ & $3.62 \times 10^{9}$ & - & $9.16 \times 10^{8}$ & $2.31 \times 10^{8}$ & $9.49 \times 10^{7}$ \\
\hline Minnesota & $4.67 \times 10^{6}$ & $9.33 \times 10^{9}$ & - & $1.98 \times 10^{7}$ & - & $2.92 \times 10^{4}$ \\
\hline Mississippi & - & - & $8.52 \times 10^{6}$ & $1.18 \times 10^{5}$ & - & $4.02 \times 10^{5}$ \\
\hline Missouri & - & - & $4.62 \times 10^{5}$ & $4.62 \times 10^{7}$ & - & $6.74 \times 10^{6}$ \\
\hline Montana & $8.13 \times 10^{5}$ & $1.63 \times 10^{9}$ & - & $6.69 \times 10^{5}$ & $6.77 \times 10^{6}$ & $2.22 \times 10^{5}$ \\
\hline
\end{tabular}




\begin{tabular}{|c|c|c|c|c|c|c|}
\hline \multirow[b]{2}{*}{$\begin{array}{c}\text { State } \\
(\operatorname{Col} \cdot A)\end{array}$} & \multicolumn{2}{|c|}{ Sugar Beets } & \multirow{2}{*}{$\begin{array}{c}\text { Peanuts } \\
1 \mathrm{~b} \\
(\mathrm{Col} \cdot \mathrm{AP})\end{array}$} & Apples & Cherries & Grapes \\
\hline & $\begin{array}{c}\text { tons } \\
(\mathrm{Col} \cdot \mathrm{AN})\end{array}$ & $\begin{array}{c}1 b \\
(\mathrm{Col} \cdot \mathrm{AO})\end{array}$ & & $\begin{array}{c}1 \mathrm{~b} \\
(\mathrm{Col} \cdot \mathrm{AQ})\end{array}$ & $\begin{array}{c}1 \mathrm{~b} \\
(\mathrm{Col} \cdot \mathrm{AR})\end{array}$ & $\begin{array}{c}1 \mathrm{~b} \\
(\mathrm{Col} \cdot \mathrm{AS})\end{array}$ \\
\hline Nebraska & $9.30 \times 10^{5}$ & $1.86 \times 10^{9}$ & - & $2.03 \times 10^{6}$ & - & $4.85 \times 10^{3}$ \\
\hline Nevada & - & - & - & $2.37 \times 10^{4}$ & - & - \\
\hline New Hampshire & - & - & - & $4.02 \times 10^{7}$ & $5.73 \times 10^{2}$ & $1.90 \times 10^{5}$ \\
\hline New Jersey & - & - & - & $9.85 \times 10^{7}$ & - & $1.07 \times 10^{6}$ \\
\hline New Mexico & $1.18 \times 10^{4}$ & $2.36 \times 10^{7}$ & $1.90 \times 10^{7}$ & $1.24 \cdot 10^{7}$ & $1.29 \times 10^{5}$ & $5.06 \times 10^{5}$ \\
\hline New York & - & - & - & $1.01 \times 10^{9}$ & $2.68 \times 10^{7}$ & $3.11 \times 10^{8}$ \\
\hline Nortin Carolina & - & - & $4.00 \times 10^{8}$ & $1.07 \times 10^{8}$ & $7.84 \times 10^{2}$ & $6.28 \times 10^{6}$ \\
\hline North Dakota & $2.53 \times 10^{6}$ & $5.06 \times 10^{9}$ & - & $7.39 \times 10^{4}$ & $5.5 \times 10^{1}$ & - \\
\hline Ohio & - & - & - & $1.32 \times 10^{8}$ & $9.56 \times 10^{5}$ & $1.51 \times 10^{7}$ \\
\hline Oklahoma & - & - & $1.59 \times 10^{8}$ & $6.27 \times 10^{6}$ & - & $6.26 \times 10^{3}$ \\
\hline Oregon & $2.64 \times 10^{5}$ & $5.28 \times 10^{8}$ & - & $1.59 \times 10^{8}$ & $8.02 \times 10^{7}$ & $8.42 \times 10^{6}$ \\
\hline Pennsyl vania & - & - & - & $4.88 \times 10^{8}$ & $7.31 \times 10^{6}$ & $9.30 \times 10^{7}$ \\
\hline Rhode Is I and & - & - & - & $5.45 \times 10^{6}$ & - & - \\
\hline South Carolina & - & - & $2.72 \times 10^{7}$ & $6.56 \times 10^{6}$ & $5.00 \times 10^{0}$ & $4.48 \times 10^{6}$ \\
\hline South Dakota & - & - & - & $5.37 \times 10^{4}$ & $1.80 \times 10^{1}$ & - \\
\hline Tennessee & - & - & $2.24 \times 10^{6}$ & $6.73 \times 10^{6}$ & $5.99 \times 10^{4}$ & $6.06 \times 10^{5}$ \\
\hline Texas & $4.86 \times 10^{5}$ & $9.72 \times 10^{8}$ & $3.24 \times 10^{8}$ & $4.45 \times 10^{5}$ & - & $8.32 \times 10^{5}$ \\
\hline Utah & - & - & - & $3.15 \times 10^{7}$ & $1.38 \times 10^{7}$ & $5.45 \times 10^{5}$ \\
\hline Vermont & - & - & - & $5.17 \times 10^{7}$ & $2.62 \times 10^{3}$ & $5.70 \times 10^{2}$ \\
\hline Virginia & - & - & $2.87 \times 10^{8}$ & $3.81 \times 10^{8}$ & $3.28 \times 10^{4}$ & $1.72 \times 10^{6}$ \\
\hline Washington & - & - & - & $2.66 \times 10^{9}$ & $9.72 \times 10^{7}$ & $2.76 \times 10^{8}$ \\
\hline West Virginia & - & - & - & $2.53 \times 10^{8}$ & $9.10 \times 10^{4}$ & $1.13 \times 10^{5}$ \\
\hline Wisconsin & - & & - & $6.73 \times 10^{7}$ & $9.40 \times 10^{6}$ & $1.69 \times 10^{5}$ \\
\hline Wyoming & $8.56 \times 10^{5}$ & $1.71 \times 10^{9}$ & - & $3.22 \times 10^{3}$ & - & - \\
\hline
\end{tabular}


'MABHL A.I (Cont'd)

\begin{tabular}{|c|c|c|c|c|c|}
\hline & Nectarines & Peaches & Pears & $\begin{array}{c}\text { Plums and } \\
\text { Prunes }\end{array}$ & $\begin{array}{l}\text { Other } \\
\text { Fruits }\end{array}$ \\
\hline $\begin{array}{l}\text { Stale } \\
(\mathrm{Col} \cdot \mathrm{A})\end{array}$ & $\begin{array}{c}l b \\
(\operatorname{Col} \cdot A T)\end{array}$ & $\begin{array}{c}\mathrm{lb} \\
(\mathrm{Col} \cdot \mathrm{AU})\end{array}$ & $\begin{array}{c}\mathrm{lb} \\
(\mathrm{Col} \cdot \mathrm{AV})\end{array}$ & $\begin{array}{c}1 \mathrm{~b} \\
(\mathrm{Col} \cdot \mathrm{AW})\end{array}$ & $\begin{array}{c}\mathrm{lb} \\
(\operatorname{Col} \cdot A X)\end{array}$ \\
\hline Alabama & $2.66 \times 10^{4}$ & $1.78 \times 10^{7}$ & $5.94 \times 10^{4}$ & $1.91 \cdot 10^{5}$ & $5.96 \times 10^{6}$ \\
\hline Al aska & - & - & - & - & $0.00 \times 10^{0}$ \\
\hline Arizona & - & $2.92 \times 10^{6}$ & $3.18 \times 10^{4}$ & $3.57 \times 10^{6}$ & $1.04 \times 10^{9}$ \\
\hline Arkansas & $1.88 \times 10^{4}$ & $1.66 \times 10^{7}$ & $2.82 \times 10^{4}$ & $7.82 \times 10^{3}$ & $8.16 \times 10^{5}$ \\
\hline California & $3.15 \times 10^{8}$ & $1.30 \times 10^{9}$ & $5.99 \times 10^{8}$ & $9.21 \times 10^{8}$ & $6.29 \times 10^{9}$ \\
\hline Colorado & $6.00 \times 10^{4}$ & $1.12 \times 10^{7}$ & $4.94 \times 10^{6}$ & $7.08 \times 10^{5}$ & $3.87 \times 10^{5}$ \\
\hline Connecticut & - & $2.43 \times 10^{6}$ & $2.50 \times 10^{6}$ & $5.93 \times 10^{4}$ & $0.00 \times 10^{0}$ \\
\hline Del aware & - & $1.99 \times 10^{6}$ & - & - & $0.00 \times 10^{0}$ \\
\hline Florida & - & $5.34 \times 10^{6}$ & $6.11 \times 10^{4}$ & $2.27 \times 10^{3}$ & $1.61 \times 10^{10}$ \\
\hline Georgia & - & $1.06 \times 10^{8}$ & $1.61 \times 1 C^{5}$ & $2.37 \times 10^{5}$ & $6.69 \times 10^{7}$ \\
\hline Hawai i & - & - & - & - & $1.21 \times 10^{8}$ \\
\hline I daho & $2.65 \times 10^{5}$ & $4.94 \times 10^{6}$ & $1.96 \times 10^{6}$ & $9.54 \times 10^{6}$ & $2.15 \times 10^{5}$ \\
\hline Illinois & - & $1.37 \times 10^{4}$ & $1.79 \times 10^{5}$ & $9.01 \times 10^{4}$ & $0.00 \times 10^{0}$ \\
\hline Indiana & - & $2.22 \times 10^{4}$ & $2.18 \times 10^{5}$ & $7.19 \times 10^{4}$ & $8.50 \times 10^{3}$ \\
\hline lowa & - & - & $1.11 \div 10^{4}$ & $7.32 \times 10^{3}$ & $3.27 \times 10^{2}$ \\
\hline Kansas & - & $4.82 \times 10^{4}$ & $4.93 \times 10^{3}$ & $4.20 \times 10^{3}$ & $1.96 \times 10^{5}$ \\
\hline Kentucky & - & $1.94 \times 10^{4}$ & $1.79 \times 10^{3}$ & $6.08 \times 10^{2}$ & $0.00 \times 10^{0}$ \\
\hline Lou is i ana & - & $3.82 \times 10^{6}$ & $8.02 \times 10^{3}$ & $8.00 \times 10^{3}$ & $3.59 \times 10^{6}$ \\
\hline Maine & - & - & $6.79 \times 10^{4}$ & $5.12 \times 10^{3}$ & $0.00 \times 10^{0}$ \\
\hline Maryl and & $6.08 \times 10^{4}$ & $1.69 \times 10^{7}$ & $4.39 \times 10^{5}$ & $1.79 \times 10^{5}$ & $2.86 \times 10^{3}$ \\
\hline Massachusetts & - & $1.44 \times 10^{6}$ & $1.44 \times 10^{6}$ & $3.19 \times 10^{4}$ & $0.00 \times 10^{0}$ \\
\hline Michigan & $1.58 \times 10^{5}$ & $4.52 \times 10^{7}$ & $1.55 \times 10^{7}$ & $1.63 \times 10^{7}$ & $5.30 \times 10^{5}$ \\
\hline Minnesola & - & - & $2.12 \times 10^{3}$ & $7.95 \times 10^{3}$ & $9.00 \times 10^{2}$ \\
\hline Mississippi & - & $2.37 \times 10^{6}$ & $8.52 \times 10^{4}$ & $2.44 \times 10^{3}$ & $1.81 \times 10^{6}$ \\
\hline Missouri & - & $4.68 \times 10^{6}$ & - & $3.37 \times 10^{3}$ & $2.84 \times 10^{5}$ \\
\hline Montana & - & - & $8.81 \times 10^{3}$ & $5.92 \times 10^{3}$ & $1.10 \times 10^{3}$ \\
\hline
\end{tabular}


'TALIเI A.l (Cont'd)

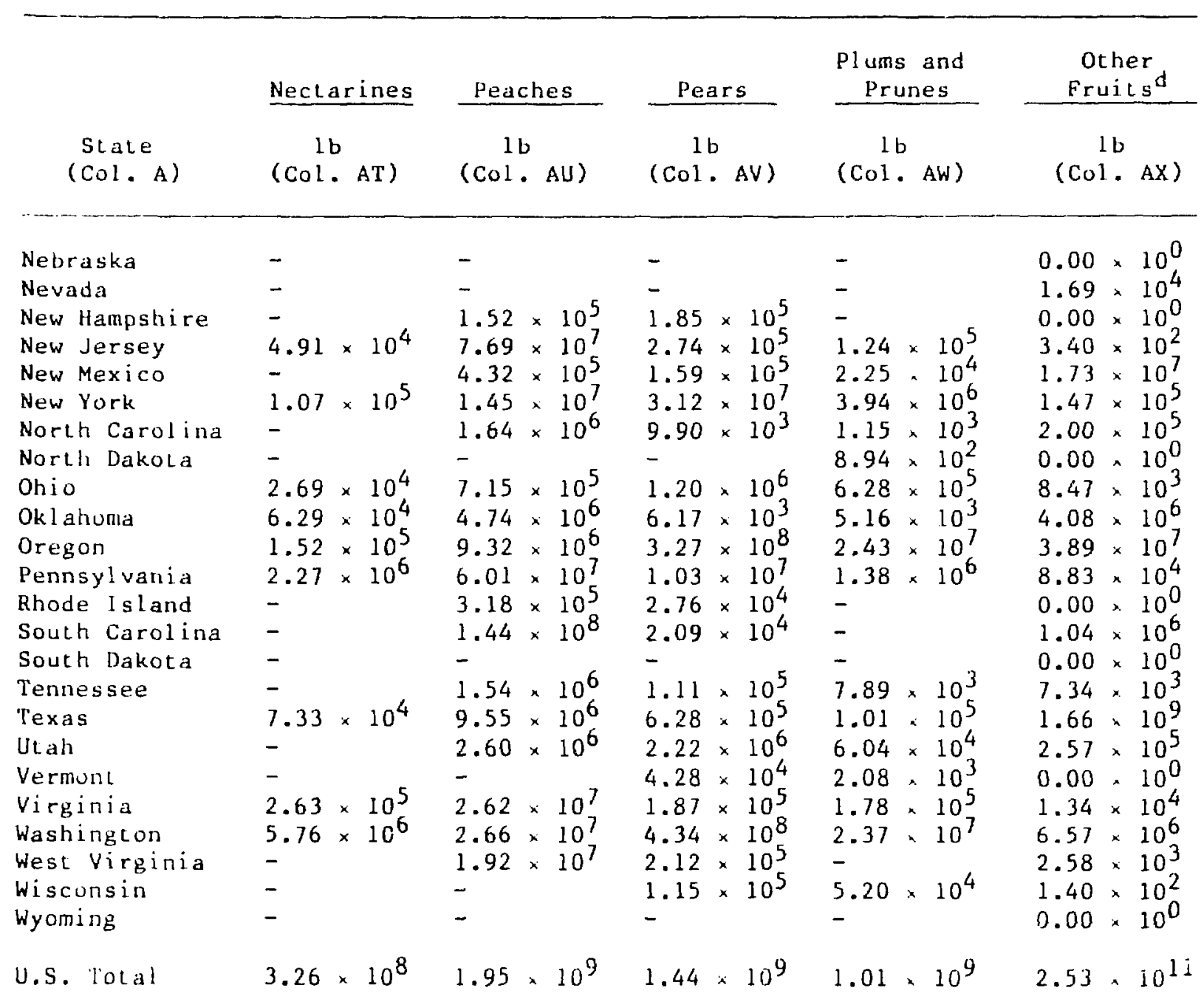




\section{'I'ABLE A.I (Cont'd)}

aushels.

$b_{A}$ hyphen indicates that the data were unpublished by the U.S. Bureau of the Census for reasons of confidentiality. In such cases, total production was very low.

${ }^{c} C_{W t}=100-1 b$ units.

Includes apricols, avocados, bananas, coffee, dales, figs, guavas, kiwifruit, mangoes, ciives, papayas, passion fruit, persimmons, pomegranates, grapefruit, kumquals, lemons, limes, oranges, Langelos, tangerines, almonds, filberts, hazelnuts, macadamia nuts, pecans, pistachios, walnuts, as well as other fruits and nuts not specifically named.

Source.: Listed in Table B.1 by spreadsheet column. 
TABLE A.2 State Agricultural Production, 1982: Meal and lggs (spreadsheet columns are provided for ease of reference to Appendix B)

\begin{tabular}{|c|c|c|c|c|c|c|}
\hline \multirow[b]{2}{*}{$\begin{array}{l}\text { State } \\
(\text { Col. A) }\end{array}$} & \multicolumn{2}{|c|}{ Cattle/Calves } & \multicolumn{2}{|c|}{ Hogs/Pigs } & \multicolumn{2}{|c|}{ Broilers } \\
\hline & $\begin{array}{l}\text { No. Sold } \\
(\text { Col. E) }\end{array}$ & $\begin{array}{c}\text { lb Slaughtered } \\
(\mathrm{Col} . \mathrm{F})\end{array}$ & $\begin{array}{l}\text { No. Sold } \\
\text { (Col. G) }\end{array}$ & $\begin{array}{l}\text { Ib Slaughtered } \\
(\mathrm{Cul} \cdot \mathrm{H})\end{array}$ & $\begin{array}{l}\text { No. Sold } \\
(\mathrm{Cr} l \cdot 1)\end{array}$ & $\begin{array}{l}\text { 1b Consumed } \\
(\mathrm{Col} \cdot \mathrm{J})\end{array}$ \\
\hline Al abama & $8.22 \times 10^{5}$ & $2.88 \times 10^{8}$ & $7.86 \times 10^{5}$ & $1.18 \times 10^{8}$ & $4.27 \times 10^{8}$ & $1.26 \times 10^{9}$ \\
\hline Al aska & $1.64 \times 10^{3}$ & $5.75 \times 10^{5}$ & $6.24 \times 10^{3}$ & $3.37 \times 10^{5}$ & $4.61 \times 10^{3}$ & $1.36 \times 10^{4}$ \\
\hline Arizona & $1.02 \times 10^{6}$ & $3.57 \times 10^{8}$ & $2.61 \times 10^{5}$ & $3.92 \times 10^{7}$ & $0.00 \times 10^{0}$ & $0.00 \times 10^{0}$ \\
\hline Arkansas & $8.22 \times 10^{5}$ & $2.88 \times 10^{8}$ & $8.27 \times 10^{5}$ & $1.24 \times 10^{8}$ & $5.69 \times 10^{8}$ & $1.68 \times 10^{9}$ \\
\hline California & $3.34 \times 10^{6}$ & $1.17 \times 10^{9}$ & $2.82 \times 10^{5}$ & $4.23 \times 10^{7}$ & $1.24 \times 10^{8}$ & $3.65 \times 10^{8}$ \\
\hline Colorado & $3.27 \times 10^{6}$ & $1.15 \times 10^{9}$ & $6.06 \times 10^{5}$ & $9.09 \times 10^{7}$ & $2.26 \times 10^{4}$ & $6.66 \times 10^{4}$ \\
\hline Connecticut & $4.75 \times 10^{4}$ & $2.66 \times 10^{7}$ & $9.20 \times 10^{3}$ & $1.38 \times 10^{6}$ & $5.40 \times 10^{5}$ & $1.59 \times 10^{6}$ \\
\hline Del aware & $2.62 \times 10^{4}$ & $9.19 \times 10^{6}$ & $1.02 \times 10^{5}$ & $1.53 \times 10^{7}$ & $1.70 \times 10^{8}$ & $5.02 \times 10^{8}$ \\
\hline Florida & $1.11 \times 10^{6}$ & $3.88 \times 10^{8}$ & $3.28 \times 10^{5}$ & $4.93 \times 10^{7}$ & $7.65 \times 10^{7}$ & $2.25 \times 10^{8}$ \\
\hline Georgia & $7.30 \times 10^{5}$ & $2.56 \times 10^{8}$ & $2.12 \times 10^{6}$ & $3.18 \times 10^{8}$ & $5.22 \times 10^{8}$ & $1.54 \times 10^{9}$ \\
\hline Hawai i & $9.17 \times 10^{4}$ & $3.21 \times 10^{7}$ & $6.69 \times 10^{4}$ & $1.00 \times 10^{7}$ & $3.17 \times 10^{6}$ & $9.36 \times 10^{6}$ \\
\hline Idaho & $1.49 \times 10^{6}$ & $5.22 \times 10^{8}$ & $1.14 \times 10^{5}$ & $1.72 \times 10^{7}$ & $1.46 \times 10^{4}$ & $4.31 \times 10^{4}$ \\
\hline Illinois & $1.53 \times 10^{6}$ & $5.37 \times 10^{8}$ & $9.53 \times 10^{6}$ & $1.43 \times 10^{9}$ & $1.38 \times 10^{5}$ & $4.08 \times 10^{5}$ \\
\hline Indiana & $8.90 \times 10^{5}$ & $3.12 \times 10^{8}$ & $7.28 \times 10^{6}$ & $1.09 \times 10^{9}$ & $1.26 \times 10^{7}$ & $3.71 \times 10^{7}$ \\
\hline I owa & $4.60 \times 10^{6}$ & $1.61 \times 10^{9}$ & $2.38 \times 10^{7}$ & $3.57 \times 10^{9}$ & $4.29 \times 10^{5}$ & $1.27 \times 10^{6}$ \\
\hline Kansas & $6.52 \times 10^{6}$ & $2.28 \times 10^{9}$ & $3.04 \times 10^{6}$ & $4.56 \times 10^{8}$ & $9.45 \times 10^{4}$ & $2.79 \times 10^{5}$ \\
\hline Kentucky & $1.22 \times 10^{6}$ & $4.28 \times 10^{8}$ & $1.39 \times 10^{6}$ & $2.09 \times 10^{8}$ & $2.41 \times 10^{6}$ & $7.11 \times 10^{6}$ \\
\hline Louisiana & $4.35 \times 10^{5}$ & $1.52 \times 10^{8}$ & $7.13 \times 10^{4}$ & $1.07 \times 10^{7}$ & $9.19 \times 10^{7}$ & $2.71 \times 10^{8}$ \\
\hline Maine & $5.24 \times 10^{4}$ & $1.84 \times 10^{7}$ & $1.21 \times 10^{4}$ & $1.81 \times 10^{6}$ & $2.01 \times 10^{7}$ & $5.91 \times 10^{7}$ \\
\hline Maryland & $1.65 \times 10^{5}$ & $5.78 \times 10^{7}$ & $3.35 \times 10^{5}$ & $5.03 \times 10^{7}$ & $2.35 \times 10^{8}$ & $6.93 \times 10^{8}$ \\
\hline Massachusetts & $4.70 \times 10^{4}$ & $1.65 \times 10^{7}$ & $4.44 \times 10^{4}$ & $6.66 \times 10^{6}$ & $1.63 \times 10^{5}$ & $4.80 \times 10^{5}$ \\
\hline Michigan & $6.42 \times 10^{5}$ & $2.25 \times 10^{8}$ & $1.73 \times 10^{6}$ & $2.60 \times 10^{8}$ & $6.01 \times 10^{5}$ & $1.77 \times 10^{6}$ \\
\hline Minnesota & $1.76 \times 10^{6}$ & $6.17 \times 10^{8}$ & $8.01 \times 10^{6}$ & $1.20 \times 10^{9}$ & $2.26 \times 10^{7}$ & $6.65 \times 10^{7}$ \\
\hline Mississippi & $7.05 \times 10^{5}$ & $2.47 \times 10^{8}$ & $3.57 \times 10^{5}$ & $5.36 \times 10^{7}$ & $2.47 \times 10^{8}$ & $7.29 \times 10^{8}$ \\
\hline Missouri & $2.50 \times 10^{6}$ & $8.75 \times 10^{8}$ & $5.89 \times 10^{6}$ & $8.85 \times 10^{8}$ & $2.15 \times 10^{7}$ & $6.33 \times 10^{7}$ \\
\hline Montana & $1.78 \times 10^{6}$ & $6.23 \times 10^{8}$ & $3.12 \times 10^{5}$ & $4.68 \times 10^{7}$ & $1.84 \times 10^{4}$ & $5.44 \times 10^{4}$ \\
\hline
\end{tabular}




\begin{tabular}{|c|c|c|c|c|c|c|}
\hline \multirow[b]{2}{*}{$\begin{array}{l}\text { State } \\
(\text { Col. A })\end{array}$} & \multicolumn{2}{|c|}{ CatLle/Calves } & \multicolumn{2}{|c|}{ Hogs/Pigs } & \multicolumn{2}{|c|}{ Broilers } \\
\hline & $\begin{array}{l}\text { No. Sold } \\
(\mathrm{Col} \cdot \mathrm{E})\end{array}$ & $\begin{array}{c}\text { lb Slaughtered } \\
(\mathrm{Col} . \mathrm{F})\end{array}$ & $\begin{array}{l}\text { No. Sold } \\
\text { (Col. C) }\end{array}$ & $\begin{array}{l}\text { Ib Slaughtered } \\
(\mathrm{Col} \cdot \mathrm{H})\end{array}$ & $\begin{array}{l}\text { No. Sold } \\
(\mathrm{Col} . \mathrm{I})\end{array}$ & $\begin{array}{l}\text { Ib Consumed } \\
(\mathrm{Col} \cdot \mathrm{J})\end{array}$ \\
\hline Nebraska & $6.01 \times 10^{6}$ & $2.11 \times 10^{9}$ & $7.06 \times 10^{6}$ & $1.06 \times 10^{9}$ & $1.04 \times 10^{5}$ & $3.07 \times 10^{5}$ \\
\hline Nevada & $2.75 \times 10^{5}$ & $9.65 \times 10^{7}$ & $1.60 \times 10^{4}$ & $2.41 \times 10^{6}$ & $5.49 \times 10^{2}$ & $1.62 \times 10^{3}$ \\
\hline New Hampstire & $2.94 \times 10^{4}$ & $1.03 \times 10^{7}$ & $9.40 \times 10^{3}$ & $1.41 \times 10^{6}$ & $2.91 \times 10^{5}$ & $8.58 \times 10^{5}$ \\
\hline New Jersey & $4.80 \times 10^{4}$ & $1.68 \times 10^{7}$ & $7.42 \times 10^{4}$ & $1.11 \times 10^{7}$ & $2.05 \times 10^{5}$ & $6.04 \times 10^{5}$ \\
\hline New Mexico & $1.15 \times 10^{6}$ & $4.03 \times 10^{8}$ & $8.50 \times 10^{4}$ & $1.28 \times 10^{7}$ & $2.48 \times 10^{3}$ & $7.32 \times 10^{3}$ \\
\hline New York & $7.47 \times 10^{5}$ & $2.62 \times 10^{8}$ & $1.83 \times 10^{5}$ & $2.75 \times 10^{7}$ & $3.81 \times 10^{5}$ & $1.12 \times 10^{6}$ \\
\hline North Carolina & $3.79 \times 10^{5}$ & $1.33 \times 10^{8}$ & $3.91 \times 10^{6}$ & $5.87 \times 10^{8}$ & $3.48 \times 10^{8}$ & $1.03 \times 10^{9}$ \\
\hline North Dakota & $1.02 \times 10^{6}$ & $3.57 \times 10^{8}$ & $4.48 \times 10^{5}$ & $6.72 \times 10^{7}$ & $3.54 \times 10^{4}$ & $1.04 \times 10^{5}$ \\
\hline Ohio & $8.73 \times 10^{5}$ & $3.06 \times 10^{8}$ & $3.57 \times 10^{6}$ & $5.37 \times 10^{8}$ & $1.16 \times 10^{7}$ & $3.43 \times 10^{7}$ \\
\hline Okl ahoma & $3.37 \times 10^{6}$ & $1.18 \times 10^{9}$ & $3.56 \times 10^{5}$ & $5.34 \times 10^{7}$ & $4.94 \times 10^{7}$ & $1.46 \times 10^{8}$ \\
\hline Oregon & $9.55 \times 10^{5}$ & $3.35 \times 10^{8}$ & $1.72 \times 10^{5}$ & $2.59 \times 10^{7}$ & $1.44 \times 10^{7}$ & $4.25 \times 10^{7}$ \\
\hline Pennsylvania & $8.85 \times 10^{5}$ & $3.10 \times 10^{8}$ & $1.67 \times 10^{6}$ & $2.51 \times 10^{8}$ & $1.04 \times 10^{8}$ & $3.06 \times 10^{8}$ \\
\hline Rhode Island & $3.54 \times 10^{3}$ & $1.24 \times 10^{6}$ & $3.21 \times 10^{3}$ & $4.91 \times 10^{5}$ & $0.00 \times 10^{0}$ & $0.00 \times 10^{0}$ \\
\hline South Carolina & $2.14 \times 10^{5}$ & $7.49 \times 10^{7}$ & $6.41 \times 10^{5}$ & $9.71 \times 10^{7}$ & $4.16 \times 10^{7}$ & $1.23 \times 10^{8}$ \\
\hline South Dakola & $2.42 \times 10^{6}$ & $8.48 \times 10^{8}$ & $3.07 \times 10^{6}$ & $4.60 \times 10^{8}$ & $2.00 \times 10^{5}$ & $5.90 \times 10^{5}$ \\
\hline Tennessee & $1.04 \times 10^{6}$ & $3.66 \times 10^{8}$ & $1.51 \times 10^{6}$ & $2.27 \times 10^{8}$ & $5.39 \times 10^{7}$ & $1.59 \times 10^{8}$ \\
\hline Texas & $1.06 \times 10^{7}$ & $3.71 \times 10^{9}$ & $9.25 \times 10^{5}$ & $1.39 \times 10^{8}$ & $1.76 \times 10^{8}$ & $5.20 \times 10^{8}$ \\
\hline Utah & $4.58 \times 10^{5}$ & $1.61 \times 10^{8}$ & $5.04 \times 10^{4}$ & $7.56 \times 10^{6}$ & $4.36 \times 10^{3}$ & $1.28 \times 10^{4}$ \\
\hline Vermont & $1.57 \times 10^{5}$ & $5.50 \times 10^{7}$ & $7.01 \times 10^{3}$ & $1.05 \times 10^{6}$ & $0.00 \times 10^{0}$ & $0.00 \times 10^{0}$ \\
\hline Virginia & $8.14 \times 10^{5}$ & $2.85 \times 10^{8}$ & $8.05 \times 10^{5}$ & $1.21 \times 10^{8}$ & $1.20 \times 10^{8}$ & $3.54 \times 10^{8}$ \\
\hline Washington & $1.13 \times 10^{6}$ & $3.95 \times 10^{8}$ & $1.17 \times 10^{5}$ & $1.76 \times 10^{7}$ & $1.69 \times 10^{7}$ & $4.98 \times 10^{7}$ \\
\hline West Virginia & $2.38 \times 10^{5}$ & $8.34 \times 10^{7}$ & $5.80 \times 10^{4}$ & $8.70 \times 10^{6}$ & $2.30 \times 10^{7}$ & $6.78 \times 10^{7}$ \\
\hline Wisconsin & $1.81 \times 10^{6}$ & $6.33 \times 10^{8}$ & $2.66 \times 10^{6}$ & $3.99 \times 10^{8}$ & $8.80 \times 10^{6}$ & $2.60 \times 10^{7}$ \\
\hline Wyoming & $9.81 \times 10^{5}$ & $3.44 \times 10^{8}$ & $5.77 \times 10^{4}$ & $8.66 \times 10^{6}$ & $2.14 \times 10^{3}$ & $6.29 \times 10^{3}$ \\
\hline U.3. Total & $7.12 \times 10^{7}$ & $2.50 \times 10^{10}$ & $9.48 \times 10^{7}$ & $1.42 \times 10^{10}$ & $3.52 \times 10^{9}$ & $1.04 \times 10^{10}$ \\
\hline
\end{tabular}




\begin{tabular}{|c|c|c|c|c|c|c|}
\hline \multirow[b]{2}{*}{$\begin{array}{l}\text { State } \\
(\text { Col. A) }\end{array}$} & \multicolumn{2}{|c|}{ Chickens } & \multicolumn{2}{|c|}{ Turkeys } & \multicolumn{2}{|c|}{ Eggs } \\
\hline & $\begin{array}{c}1000 \text { lb Sold } \\
(\mathrm{Col} \cdot \mathrm{K})\end{array}$ & $\begin{array}{l}\text { 1b Consumed } \\
(\mathrm{Col} . \mathrm{L})\end{array}$ & $\begin{array}{l}\text { No. Sold } \\
(\mathrm{Col} \cdot \mathrm{M})\end{array}$ & $\begin{array}{l}1 \mathrm{~b} \text { Consumed } \\
(\mathrm{Co} 1 \cdot \mathrm{N})\end{array}$ & $\begin{array}{l}\text { Millions } \\
(\mathrm{Col} \cdot \mathrm{O})\end{array}$ & $\begin{array}{c}\mathrm{lb} \\
(\mathrm{Col} \cdot \mathrm{P})\end{array}$ \\
\hline Al abama & $6.98 \times 10^{4}$ & $4.31 \times 10^{7}$ & $0.00 \times 10^{0}$ & $0.00 \times 10^{0}$ & $2.88 \times 10^{3}$ & $3.77 \times 10^{8}$ \\
\hline laska & $2.71 \times 10^{2}$ & $1.67 \times 10^{5}$ & $1.65 \times 10^{2}$ & $2.52 \times 10^{3}$ & $8.80 \times 10^{0}$ & $1.15 \times 10^{6}$ \\
\hline Arizona & $9.53 \times 10^{2}$ & $5.89 \times 10^{5}$ & $8.91 \times 10^{3}$ & $1.36 \times 10^{5}$ & $1.14 \times 10^{2}$ & $1.49 \times 10^{7}$ \\
\hline Arkansas & $1.22 \times 10^{5}$ & $7.53 \times 10^{7}$ & $1.36 \times 10^{7}$ & $2.08 \times 10^{8}$ & $4.06 \times 10^{3}$ & $5.32 \times 10^{8}$ \\
\hline Cal ifornia & $8.92 \times 10^{4}$ & $5.51 \times 10^{7}$ & $2.11 \times 10^{7}$ & $3.22 \times 10^{8}$ & $8.29 \times 10^{3}$ & $1.08 \times 10^{9}$ \\
\hline Colorado & $5.69 \times 10^{3}$ & $3.51 \times 10^{6}$ & $0.00 \times 10^{0}$ & $0.00 \times 10^{0}$ & $6.27 \times 10^{2}$ & $8.20 \times 10^{7}$ \\
\hline Connecticur & $1.32 \times 10^{4}$ & $8.18 \times 10^{6}$ & $0.00 \times 10^{0}$ & $0.00 \times 10^{0}$ & $1.06 \times 10^{3}$ & $1.38 \times 10^{8}$ \\
\hline Delaware & $7.31 \times 10^{3}$ & $4.52 \times 10^{6}$ & $0.00 \times 10^{0}$ & $0.00 \times 10^{0}$ & $1.37 \times 10^{2}$ & $1.79 \times 10^{7}$ \\
\hline Florida & $3.71 \times 10^{4}$ & $2.29 \times 10^{7}$ & $7.37 \times 10^{2}$ & $1.13 \times 10^{4}$ & $2.96 \times 10^{3}$ & $3.88 \times 10^{8}$ \\
\hline Georgia & $1.15 \times 10^{5}$ & $7.13 \times 10^{7}$ & $2.99 \times 10^{6}$ & $4.58 \times 10^{7}$ & $5.36 \times 10^{3}$ & $7.01 \times 10^{8}$ \\
\hline Hawai i & $1.50 \times 10^{3}$ & $9.24 \times 10^{5}$ & $0.00 \times 10^{0}$ & $0.00 \times 10^{0}$ & $2.02 \times 10^{2}$ & $2.65 \times 10^{7}$ \\
\hline Idaho & $2.47 \times 10^{3}$ & $1.53 \times 10^{6}$ & $0.00 \times 10^{0}$ & $0.00 \times 10^{0}$ & $2.38 \times 10^{2}$ & $3.11 \times 10^{7}$ \\
\hline Illinois & $1.39 \times 10^{4}$ & $8.60 \times 10^{6}$ & $0.00 \times 10^{0}$ & $0.00 \times 10^{0}$ & $1.16 \times 10^{3}$ & $1.52 \times 10^{8}$ \\
\hline Indiana & $5.57 \times 10^{4}$ & $3.44 \times 10^{7}$ & $0.00 \times 10^{0}$ & $0.00 \times 10^{0}$ & $4.46 \times 10^{3}$ & $5.84 \times 10^{8}$ \\
\hline Iowa & $2.79 \times 10^{4}$ & $1.72 \times 10^{7}$ & $7.22 \times 10^{6}$ & $1.10 \times 10^{8}$ & $1.98 \times 10^{3}$ & $2.60 \times 10^{8}$ \\
\hline Kansas & $7.36 \times 10^{3}$ & $4.55 \times 10^{6}$ & $0.00 \times 10^{0}$ & $0.00 \times 10^{0}$ & $4.62 \times 10^{2}$ & $6.04 \times 10^{7}$ \\
\hline Kentucky & $6.55 \times 10^{3}$ & $4.05 \times 10^{6}$ & $4.12 \times 10^{3}$ & $6.31 \times 10^{4}$ & $4.84 \times 10^{2}$ & $6.33 \times 10^{7}$ \\
\hline Louisiana & $1.51 \times 10^{4}$ & $9.31 \times 10^{6}$ & $2.24 \times 10^{2}$ & $3.43 \times 10^{3}$ & $4.57 \times 10^{2}$ & $5.98 \times 10^{7}$ \\
\hline Maine & $3.25 \times 10^{4}$ & $2.01 \times 10^{7}$ & $0.00 \times 10^{0}$ & $0.00 \times 10^{0}$ & $1.43 \times 10^{3}$ & $1.87 \times 10^{8}$ \\
\hline Mary-and & $1.36 \times 10^{4}$ & $8.40 \times 10^{G}$ & $0.00 \times 10^{0}$ & $0.00 \times 10^{0}$ & $6.58 \times 10^{2}$ & $8.61 \times 10^{7}$ \\
\hline Massachusetts & $4.89 \times 10^{3}$ & $3.02 \times 10^{6}$ & $1.54 \times 10^{5}$ & $2.35 \times 10^{6}$ & $3.14 \times 10^{2}$ & $4.11 \times 10^{7}$ \\
\hline Michigan & $2.05 \times 10^{4}$ & $1.26 \times 10^{7}$ & $1.85 \times 10^{6}$ & $2.83 \times 10^{7}$ & $1.53 \times 10^{3}$ & $2.00 \times 10^{8}$ \\
\hline Minnesota & $3.69 \times 10^{4}$ & $2.28 \times 10^{7}$ & $2.75 \times 10^{7}$ & $4.20 \times 10^{8}$ & $2.43 \times 10^{3}$ & $3.18 \times 10^{8}$ \\
\hline Mississippi & $3.99 \times 10^{4}$ & $2.46 \times 10^{7}$ & $1.37 \times 10^{2}$ & $2.10 \times 10^{3}$ & $1.52 \times 10^{3}$ & $1.99 \times 10^{8}$ \\
\hline Missouri & $3.71 \times 10^{4}$ & $2.29 \times 10^{7}$ & $9.87 \times 10^{6}$ & $1.51 \times 10^{8}$ & $1.46 \times 10^{3}$ & $1.90 \times 10^{8}$ \\
\hline Montana & $3.44 \times 10^{3}$ & $2.12 \times 10^{6}$ & $1.01 \times 10^{4}$ & $1.54 \times 10^{5}$ & $1.88 \times 10^{2}$ & $2.46 \times 10^{7}$ \\
\hline Nebraska & $6.08 \times 10^{3}$ & $3.76 \times 10^{6}$ & $7.29 \times 10^{5}$ & $1.12 \times 10^{7}$ & $8.09 \times 10^{2}$ & $1.06 \times 10^{8}$ \\
\hline
\end{tabular}




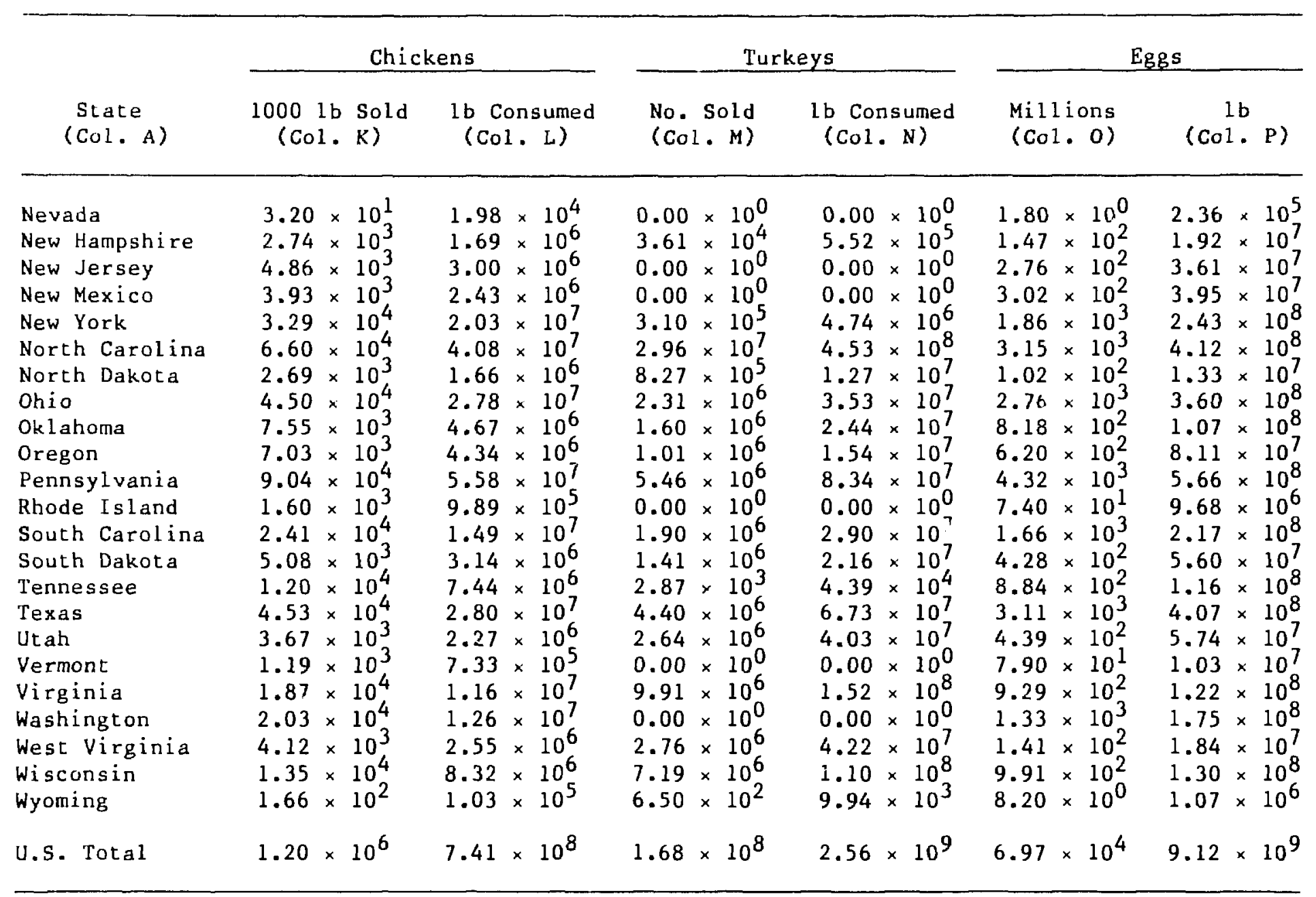

Source: Listed in Table B.I by spreadsheet column. 


\section{$55 / 56$}

APPENDIX B:

AGRICULTURAL PRODUCTION STATISTICS DOCUMENTATION 


\section{APPENDIX B:}

\section{AGRICULTURAL PRODUCTION STATISTICS DOCUMENTATION}

Tables B.1-B.3 identify the sources and/or derivation formulas for the data entered into each column of the agricultural statistics spreadsheets. Actual data values are given in Appendix A.

TABLE B.1 Documentation for Basic Agricultural Production Statistics ${ }^{\mathbf{a}}$

\begin{tabular}{|c|c|c|}
\hline Col umn & Description & Data Source ${ }^{b}$ or Formula \\
\hline A & State names & \\
\hline B & Land in state (acres) & Census Table 1, PP. $134-140$ \\
\hline $\mathrm{C}$ & Percent land in farms & Census Table 1, Pp. 134-140 \\
\hline$D$ & Dairy & SRS Statistical Bulletin 722, p. 22 \\
\hline$E$ & Cattle/calves (no. sold) & Census Table 11 , pp. 218-224 \\
\hline$F$ & Cattle/calves ( $1 \mathrm{~b}$ slaughtered) & Col. $E \times 55.13290366 \% \times 635.661 b$ \\
\hline G & Hogs/pigs (no. sold) & Census Table 12, pp. $232-238$ \\
\hline $\mathrm{H}$ & Hogs/pigs (1b slaughtered) & Col. G $\times 171.761 \mathrm{~b} \times 87.4029372 \%$ \\
\hline I & Broilers (no. sold) & Census Table 14, pp. 243-249 \\
\hline $\mathrm{J}$ & Broilers ( 1 b consumed) & Col. I $\times 4.03 \mathrm{Lb} \times 73.1565 \%$ \\
\hline k & Chickens ( $1000 \mathrm{lb}$ sold) & SRS Statistical Bulletin $725,0.14$ \\
\hline L & Chickens (lb consumed) & Col. $K \times 1000 \times 61.7731747 \%$ \\
\hline M & Turkeys (no. sold) & Census Table 14, pp. 243-249 \\
\hline $\mathrm{N}$ & Turkeys ( 1 b consumed) & Col. $\mathrm{M} \times 19.191 \mathrm{~b} \times 79.701 \%$ \\
\hline 0 & Eggs (millions) & SRS Statistical Bulletin 721 , p. 17 \\
\hline $\mathrm{P}$ & Eggs (1b) & $\begin{array}{l}\text { Col. } 0 \times 1,000,000 \times 1.571 b / 12 \\
(\text { dozen) }\end{array}$ \\
\hline$Q$ & Corn (bu) & Census Table 15, pp. $250-262$ \\
\hline $\mathrm{R}$ & Corn $(1 b)$ & $\mathrm{Col} \cdot \mathrm{Q} \times 56 \mathrm{lb} / \mathrm{bu}$ \\
\hline$S$ & Wheat (bu) & Census Table 15, pp. $250-262$ \\
\hline I & Wheat ( $1 \mathrm{~b})$ & Col. $\mathrm{S} \times 60 \mathrm{lb} / \mathrm{bu}$ \\
\hline U & Soybeans (bu) & Census Table $1 \%$, pp. $250-262$ \\
\hline V & Soybeanș (1b) & Col. U $\times 60 \mathrm{lb} / \mathrm{bu}$ \\
\hline in & Dotatoes (cwt) & Census Table 15 , pp. $250-262$ \\
\hline$x$ & Potatoes ( $1 b)$ & Col. $W \times 100$ \\
\hline Y & Honey (1b) & Census Table 20, p. 298 \\
\hline Z & Sorghum (bu) & Census Table 24, pp. $311-312$ \\
\hline AA & Sorghum (1.b) & $\mathrm{Col} \cdot \mathrm{Z} \times 56 \mathrm{lb} / \mathrm{bu}$ \\
\hline$A B$ & Barley (bu) & Census Table 24, pp. $3 i 2-313$ \\
\hline $\mathrm{AC}$ & Barley (Lb) & $\mathrm{Col} \cdot \mathrm{AB} \times 48 \mathrm{lb} / \mathrm{bu}$ \\
\hline$A D$ & Oats (bu) & Census Table 24, pp. 312-315 \\
\hline$A E$ & Oats (1b) & $\mathrm{Coi} \cdot \mathrm{AD} \times 32 \mathrm{lb} / \mathrm{bu}$ \\
\hline
\end{tabular}


TABLE B.1 (Cont'd)

Column Descripion Data Source ${ }^{b}$ or Formula

\begin{tabular}{|c|c|c|}
\hline $\mathrm{AF}$ & Rye (bu) & Census Table 24, p. 316 \\
\hline $2 G$ & Rye (1b) & Col. $A E \times 561 b / b u$ \\
\hline $\mathrm{AH}$ & Sunflowers ( $\mathrm{Lb}$ ) & Census Table 24, p. 317 \\
\hline AI & Dry beans (cwt) & Census Table 25 , p. 319 \\
\hline AJ & Dry beans ( $1 b)$ & $\mathrm{Col} \cdot \mathrm{AI} \times 100$ \\
\hline $\pm K$ & Dry peas ( $1 \mathrm{~b})$ & Census rible 25 , p. 319 \\
\hline$A L$ & Sweet potatoes (bu) & Census Table 25, p. 321 \\
\hline AM & Sweet potatoes (1b) & $C c \therefore$ AL $\times 551 \mathrm{~b} / \mathrm{bu}$ \\
\hline$\Delta \mathrm{N}$ & Sugar beets (rons) & Census Table 25 , p. 321 \\
\hline 20 & Sugar beets ( $1 \mathrm{~b})$ & Col. AN $\times 2000$ \\
\hline $\mathrm{AP}$ & Peanuts ( $1 b)$ & Census Table 25 , p. 322 \\
\hline$A Q$ & Apples (1b) & Census Table 28 , p. 359 \\
\hline$A R$ & Cherries ( $1 \mathrm{~b}$ ) & Census Table 28, p. 360 \\
\hline AS & Grapes (1b) & Census Table 28 , pp. $361-362$ \\
\hline AT & Nectarines (lb) & Census Table 28 , p. 362 \\
\hline $\mathrm{AU}$ & Peaches ( $1 b$ ) & Census Table 28, p. 363 \\
\hline AV & Pears (Ib) & Census Table 28, p. 364 \\
\hline $\mathrm{AW}$ & Plums and prunes ( $1 b$ ) & Census Table 28 , p. 365 \\
\hline$A X$ & Other fruits (lb) & Census Table 28, pp. 359-369 \\
\hline AY & State names & \\
\hline$A Z$ & Total crops (1b) & $\begin{array}{l}\text { Cols. }+\mathrm{C}+\mathrm{V}+\mathrm{X}+\mathrm{Y}+\mathrm{A}+ \\
\mathrm{AC}+\mathrm{AE}+\mathrm{AG}+\mathrm{AH}+\mathrm{AJ}+\mathrm{AK}+ \\
\mathrm{AM}+\mathrm{AO}+\mathrm{AP}+\mathrm{AQ}+\mathrm{AR}+\mathrm{AS}+ \\
\mathrm{AT}+\mathrm{AU}+\mathrm{AV}+\mathrm{AW}+\mathrm{AX}\end{array}$ \\
\hline $\mathrm{BA}$ & Total meat (lb) & Cols. $\mathrm{F}+\mathrm{H}+\mathrm{J}+\mathrm{L}+\mathrm{N}+\mathrm{P}$ \\
\hline $\mathrm{BB}$ & Total dairy (lb) & Col. D $\times 10^{6}$ \\
\hline
\end{tabular}

${ }^{a}$ Basic agricultural production statistics are presented in Tables 3.1 , A.I, and A.2 of this report.

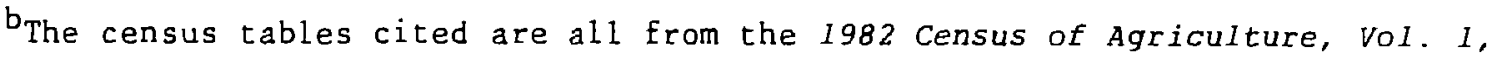
Geographic Series, Part 51, United States Summary and State Data, U.S. Bureau of the Census, AC82-A-51 (OCt. 1984). The three statistical bulletins cited were all published by the USDA Statistical Reporting Service (SRS), Crop Rotating Board. Th: three c ted are (1) Bullet in 722: Milk inal Estimates 1979-82 (Feb. 1985), (2) Bulletin 721: Chicken and Eggs, Final Esti rates for 1980-83 (Jan. 1985), and (3) Bulletin 725: Poultry ?roduction, Disposition and Income Final sstimates for 1980-83 (April 1985). 
TABLE B.2 Documentation for Agricultural Production for Lund in Farms ${ }^{\mathrm{a}}$

\begin{tabular}{|c|c|c|}
\hline Col umn & Description & Formula \\
\hline $\mathrm{BC}$ & State names & \\
\hline $\mathrm{BD}$ & Crops ( $1 \mathrm{~b} / \mathrm{acre})$ & $\mathrm{Col} \cdot \mathrm{AZ} /[\mathrm{Col} \cdot \mathrm{B} \times(\mathrm{Col} \cdot \mathrm{C} / \mathrm{l00})]$ \\
\hline$B E$ & Meat (1biacre) & $\mathrm{Cc} \mathrm{C}_{2}, \mathrm{BA} /[\mathrm{Col}, \mathrm{B} \times(\mathrm{Col}, \mathrm{C} / 100)]$ \\
\hline$B E$ & Dairy (1b/acre) & $\mathrm{Col} \cdot \mathrm{BB} /[\mathrm{Col} \cdot \mathrm{B} \times(\mathrm{Col} \cdot \mathrm{C} / 100)]$ \\
\hline $\mathrm{BC}$ & State names & \\
\hline $\mathrm{BH}$ & Crops $\left(\mathrm{kg} / \mathrm{km}^{2}\right)$ & $\begin{array}{l}(\mathrm{Col} \cdot \mathrm{AZ} \times 0.45359237 \mathrm{~kg} / \mathrm{lb}) /[\mathrm{Col} \cdot \mathrm{B} \\
\left.\times(\mathrm{Col} \cdot \mathrm{C} / 100) \times 0.0040468564 \mathrm{~km}^{2} / \mathrm{acre}\right]\end{array}$ \\
\hline BI & Meat $\left(\mathrm{kg} / \mathrm{km}^{2}\right)$ & $\begin{array}{l}(\mathrm{Col} \cdot \mathrm{BA} \times 0.45359237 \mathrm{~kg} / \mathrm{lb}) /[\mathrm{Col} \cdot \mathrm{B} \\
\left.\times(\mathrm{Col} \cdot \mathrm{C} / 100) \times 0.0040468564 \mathrm{~km}^{2} / \mathrm{acre}\right]\end{array}$ \\
\hline $\mathrm{BJ}$ & Dairy $\left(\mathrm{kg} / \mathrm{km}^{2}\right)$ & $\begin{array}{l}(\mathrm{Col} \cdot \mathrm{BB} \times 0.45359237 \mathrm{~kg} / \mathrm{lb}) /[\mathrm{Col} \cdot \mathrm{B} \\
\left.\times(\mathrm{Col} \cdot \mathrm{C} / 100) \times 0.0040468564 \mathrm{~km}^{2} / \mathrm{acre}\right]\end{array}$ \\
\hline
\end{tabular}

${ }^{a}$ Agricultural production statistics for land in farms are presented in Table 3.2 of this report.

TABLE B.3 Documentation for Agricultural Production for Total Land in State ${ }^{\mathrm{a}}$

\begin{tabular}{|c|c|c|}
\hline Column & Description & Formula for Data Entered \\
\hline BK & State names & \\
\hline $\mathrm{BL}$ & Crops (Ib/acre) & Col. AZ/Col. B \\
\hline $\mathrm{BM}$ & Meat (lb/acre) & Col. BA/Col. B \\
\hline $\mathrm{BN}$ & Dairy (lb/acre) & $\mathrm{Col} \cdot \mathrm{BB} / \mathrm{Col} \cdot \mathrm{B}$ \\
\hline BO & State names & \\
\hline $\mathrm{BP}$ & Crops $\left(\mathrm{kg} / \mathrm{km}^{2}\right)$ & $\begin{array}{l}(\text { Col. } \mathrm{AZ} \times 0.45359237 \mathrm{~kg} / \mathrm{lb}) / \\
\left(\text { Col. } \mathrm{B} \times 0.0040468564 \mathrm{~km}^{2} / \text { acre }\right)\end{array}$ \\
\hline $\mathrm{BQ}$ & Meat $\left(\mathrm{kg} / \mathrm{km}^{2}\right)$ & $\begin{array}{l}(\mathrm{Col} . \mathrm{BA} \times 0.45359237 \mathrm{~kg} / \mathrm{lb}) / \\
\left(\mathrm{Col} . \mathrm{B} \times 0.0040468564 \mathrm{~km}^{2} / \mathrm{acre}\right)\end{array}$ \\
\hline $\mathrm{BR}$ & Dairy $\left(\mathrm{kg} / \mathrm{km}^{2}\right)$ & $\begin{array}{l}(\mathrm{CoL} \cdot \mathrm{BB} \times 0.45359237 \mathrm{~kg} / \mathrm{lb}) / \\
\left(\mathrm{CoL} \cdot \mathrm{B} \times 0.0040468564 \mathrm{~km}^{2} / \text { acre }\right)\end{array}$ \\
\hline
\end{tabular}

agricultural production statistics for Land in farms are presented in Table 3.3 of this report. 